\title{
ANALYZING GLOBAL CYBER ATTACK CORRELATES THROUGH AN OPEN DATABASE
}

\author{
A Thesis \\ presented to \\ the Faculty of California Polytechnic State University, \\ San Luis Obispo
}

In Partial Fulfillment

of the Requirements for the Degree

Master of Science in Computer Science

by

Brady Aiello

June 2018 
(c) 2018

Brady Aiello

ALL RIGHTS RESERVED 


\section{COMMITTEE MEMBERSHIP}

TITLE:

Analyzing Global Cyber Attack Correlates

Through an Open Database

AUTHOR: Brady Aiello

DATE SUBMITTED: June 2018

COMMITTEE CHAIR: Bruce Debruhl, Ph.D.

Associate Professor of Computer Science

COMMITTEE MEMBER: Foaad Khosmood, Ph.D.

Associate Professor of Computer Science

COMmitTeE MEMBER: Franz Kurfess, Ph.D.

Professor of Computer Science 


\begin{abstract}
Analyzing Global Cyber Attack Correlates Through an Open Database
\end{abstract}

Brady Aiello

As humanity becomes more reliant on digital storage and communication for every aspect of life, cyber attacks pose a growing threat. However, cyber attacks are generally understood as individual incidents reported in technological circles, sometimes tied to a particular vulnerability. They are not generally understood through the macroscopic lens of statistical analysis spanning years over several countries and sectors, leaving researchers largely ignorant of the larger trends and correlates between attacks. This is large part due to the lack of a coherent and open database of prominent attacks. Most data about cyber attacks has been captured using a repository of common vulnerabilities and exposures (CVE's), and "honey pots", unsecured internet-connected devices which record attacks as they occur against them. These approaches help in the process of identifying vulnerabilities, but they do not capture the real world impact these attacks achieve. Therefore, in this thesis I create a database of 4,000 cyber attacks using a semi-open data source, and perform analytical queries on it to gather insights into how cyber attack volume varies among countries and sectors, and the correlates of cyber attack victims. From here, it is also possible to relate socio-economic data such as GDP and World Happiness Index to cyber attack volume. The end result is an open database of cyber attacks that allows researchers to understand the larger underlying forces which propel cyber attacks. 


\section{ACKNOWLEDGMENTS}

I would like to thank Cal Poly for the opportunity to study computer science, and all of their generous scholarships which made this work possible. I would also like to thank Cisco for funding our research of security in the Internet of Things. Thank you to my committee. Professors Khosmood and Kurfess gave me helpful feedback that has made this work much stronger. Thank you to my advisor, Professor DeBruhl, who has helped me find direction and focus on what is important in this thesis. Thank you to Paolo Passeri, whose data is the backbone of this research. Without your meticulously assembled open data, none of this work is possible. Thank you to my parents who encouraged me to pursue my dreams. And lastly, thank you to my wife Erin and my son, Khaver for bearing with the difficult demands of our higher education for so many years. You are the best family anyone could hope to have. 


\section{TABLE OF CONTENTS}

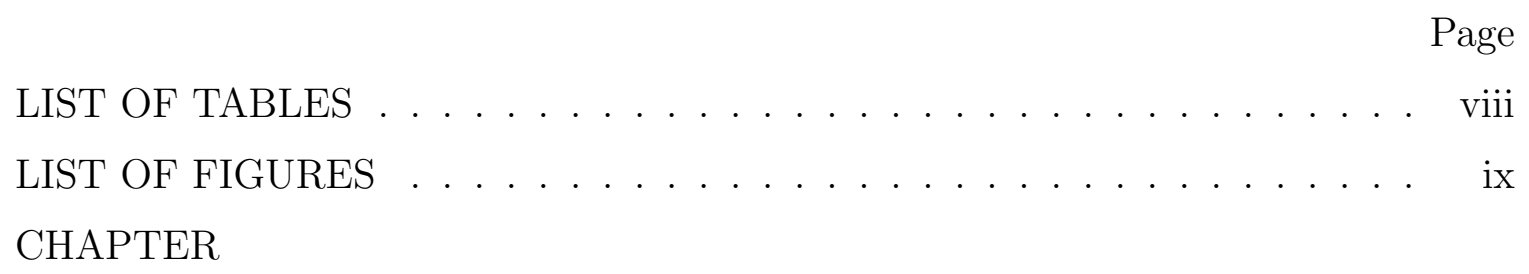

1 Introduction . . . . . . . . . . . . . . . . . 1

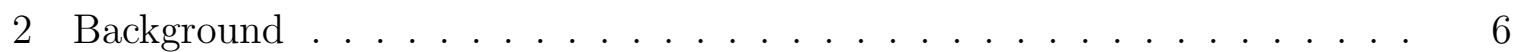

3 The Cyber Attack Database Implementation . . . . . . . . . . . . . . . 11

3.1 Open Data on Cyber Attacks . . . . . . . . . . . . . 11

3.2 Hackmageddon . . . . . . . . . . . . . . . . . . 12

3.3 Making the Spreadsheets SQL-Friendly . . . . . . . . . . . . . 15

3.4 Creating the Cyber Attack Database . . . . . . . . . . . 16

3.5 Inserting The Data . . . . . . . . . . . . . . 16

3.6 Querying The Data . . . . . . . . . . . . . . 18

3.7 Analysis Overview . . . . . . . . . . . . . . . . . . . . 19

4 Capturing Socio-economic Data . . . . . . . . . . . . . 23

4.1 The World Happiness Report . . . . . . . . . . . . . . . . . 23

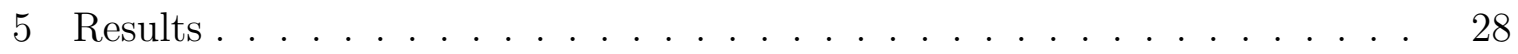

5.1 By Country . . . . . . . . . . . . . . . . 30

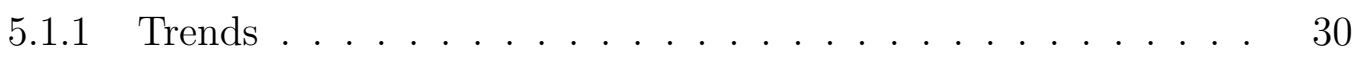

5.1 .2 Correlates ...................... 36

5.2 By Sector . . . . . . . . . . . . . . . . . . . . 42

5.3 Socioeconomic Factors . . . . . . . . . . . . . . 51

5.4 Attack Classes . . . . . . . . . . . . . . . . 54

5.5 Attack Vectors . . . . . . . . . . . . . 56

5.6 Apriori Results . . . . . . . . . . . . . . . 57

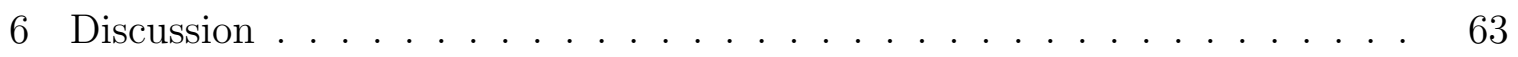

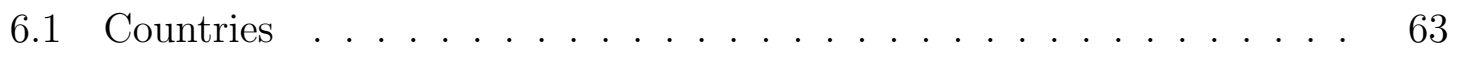

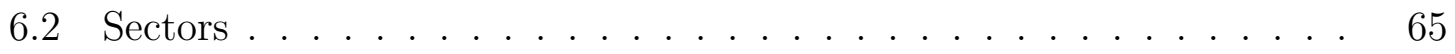

6.3 Socioeconomic Factors . . . . . . . . . . . . . . . 67 
6.4 Attack Classes . . . . . . . . . . . . . . . . . . . . . 69

6.5 Attack Vectors . . . . . . . . . . . . . . . . . . 69

7 Conclusion . . . . . . . . . . . . . . . . . . . . 70

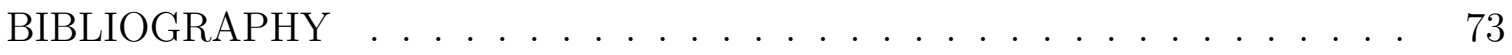
APPENDICES

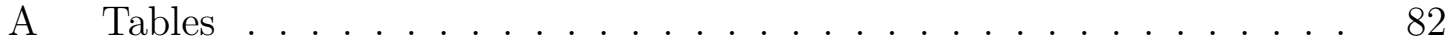




\section{LIST OF TABLES}

Table

3.1 The "Attacks" Spreadsheet . . . . . . . . . . . . . 14

3.2 The "attacks" schema . . . . . . . . . . . . . . . . 17

3.3 Total Attacks By Country . . . . . . . . . . . . . . 21

3.4 Interpreting the Pearson correlation coefficient . . . . . . . . . . 22

4.1 Annual GDP Correlates of Total Cyber Attack Volume Received 2014-2017 ........................ 26

5.1 Target Country to Target Country Correlation . . . . . . . . . . 43

5.2 Most targeted sectors . . . . . . . . . . . . . . . 45

5.3 Cross Industry Attack Correlations . . . . . . . . . . . . 59

5.4 Socio-Economic Correlates of Total Cyber Attack Volume Received 60

5.5 Socio-Economic Correlates of Total Cyber Attack Volume Received Excluding US . . . . . . . . . . . . . . . . . . . 61

$5.6 \quad$ Attack Types . . . . . . . . . . . . . . . . . 62

6.1 Change in Attacks By Sector . . . . . . . . . . . . 66

6.2 Types of Attacks Against Individuals . . . . . . . . . . . . . 67

6.3 Types of Attacks Against Industry . . . . . . . . . . . . . 67

6.4 Types of Attacks Against Governments . . . . . . . . . . . . 68

A.1 Target Country to Target Country Correlation . . . . . . . . . . . . 82

A.2 Apriori Rules Associations 1 to ${ }^{*} \ldots \ldots$. . . . . . . . . . 90

A.3 Apriori Rules Associations 2 to * . . . . . . . . . . . . . . . . 91

A.4 Apriori Rules Associations 3 to ${ }^{*} \ldots \ldots$. . . . . . . . . 92 


\section{LIST OF FIGURES}

Figure

Page

1.1 IoT Device Growth . . . . . . . . . . . . . . . . . . . 3

$3.1 \quad$ Attacks as a Pandas dataframe . . . . . . . . . . . . 18

5.1 Total Attacks Timeline with Rolling Average . . . . . . . . . . . . 31

5.2 US Attacks Timeline . . . . . . . . . . . . . . . . 32

5.3 Attacks by Country Timeline - Timeline of cyber attack volume by country for top 5 victims. . . . . . . . . . . . . . . . . 32

5.4 Attacks Timeline for Great Britain and India . . . . . . . . . . . 33

5.5 Attacks Timeline for Canada and Australia . . . . . . . . . . . 34

5.6 Attacks Timeline for Russia and Israel . . . . . . . . . . . . . . . 34

5.7 Average Attacks / Month US . . . . . . . . . . . . . 35

5.8 Average Attacks / Month Great Britain . . . . . . . . . . . 35

5.9 Average Attacks / Month for India and Pakistan . . . . . . . . . 36

5.10 Average Attacks / Month for Canada and Australia . . . . . . . . . 36

5.11 Attacks Timeline for Turkey and the Phillipines . . . . . . . . 38

5.12 Attacks Timeline for Canada and Pakistan . . . . . . . . . . . 39

5.13 Attacks Timeline for Japan and China . . . . . . . . . . . . . . . 39

5.14 Attacks Timeline for India and Pakistan . . . . . . . . . . . . . . 40

5.15 Attacks Timeline for Italy and Germany . . . . . . . . . . . . . . . 41

5.16 Attacks Timeline for the US and China . . . . . . . . . . . . 41

5.17 Attacks Timeline for Industry and Org Sectors _ . . . . . . . . . 44

5.18 Attacks Timeline for $>1$ Label . . . . . . . . . . . . . . 44

5.19 ISIC Compliant Target Classes . . . . . . . . . . . . 46

5.20 Attacks Timeline for Attacks Against Individuals and Industry Sectors 47

5.21 Attacks Timeline for Individuals and Health Care . . . . . . . . . 49

5.22 Attacks Timeline for Government and Industry Sectors . . . . . . . 49

5.23 Attacks Timeline for Government and Individuals . . . . . . . . 50

5.24 Attacks Timeline for Individuals and Finance . . . . . . . . . . . 50 
5.25 Attacks Timeline for All Attack Classes _. . . . . . . . . . . 55

5.26 Attacks Timeline for All Attack Types, 6 month rolling average . . 57 


\section{Chapter 1}

\section{INTRODUCTION}

According to a 2017 study by Cybersecurity Ventures:

Cybercrime is the greatest threat to every company in the world, and one of the biggest problems with mankind. The impact on society is reflected in the numbers.

Last year, Cybersecurity Ventures predicted that cybercrime will cost the world $\$ 6$ trillion annually by 2021, up from $\$ 3$ trillion in 2015 . This represents the greatest transfer of economic wealth in history, risks the incentives for innovation and investment, and will be more profitable than the global trade of all major illegal drugs combined [57].

According to a study by Hiscox Insurance on cyber attacks in 2017 across the US, Great Britain, Spain, Germany, and the Netherlands, involving 4,103 organizations in private and public sectors, the average annual cost due to cyber attacks to a single business is $\$ 229,000$, and $\% 73$ of organizations were not prepared for a cyber attack [47]. For organizations with more than 1,000 employees, the average annual cost of total cyber incidents was $\$ 356,000$ in Spain, and $\$ 1.05 \mathrm{M}$ in the US [47]. The largest firms in the US lost $\$ 25 \mathrm{M}$ annually to cyber crime, while the largest firms in the Great Britain and Germany lost $\$ 20 \mathrm{M}[47]$. Businesses with fewer than 100 employees lost between $\$ 24,000$ on average in Spain to $\$ 63,000$ on average in Germany [47]. The greatest cost for a single incident ranged from $\$ 800,000$ in Spain to $\$ 5 \mathrm{M}$ in Germany, and the US in the middle at $\$ 2 \mathrm{M}[47]$. Cyber crime presents a substantial and growing threat to both the private and public sector.

Cyber security incidents also cost individual consumers. The WannaCry attack 
of May 2017 affected 300,000 machines, and cost \$4B [47]. The attack, carried out by North Korea, disabled the machines of users all around the world, promising to free them for a ransom, an instance known as "ransomware" [34]. The attacks hit hospitals in Great Britain especially hard, impeding medical work, and risking lives [34]. In September 2017, Equifax experienced a data breach exposing the information of 145 million Americans, and some citizens of Great Britain and Canada, exposing their Social Security numbers, dates of birth, driver's license numbers, driver's license dates and states, home addresses, and credit card numbers [29].

As computers become more ingrained in human life, cyber attacks even have the capacity to kill. In 2017, a pacemaker model implanted in 465,000 people was recalled over concerns about holes in its security that could let a hacker drain the battery or alter the heart's rhythm arbitrarily [45]. In July 2017, a security researcher demonstrated how a popular internet-connected car wash system could be commandeered from anywhere in the world to attack anyone inside the car wash [65]. Full administrator privileges were granted by entering the default password " $12345 "$ [65].

Car washes are one example of large equipment connected to the internet. However, in industrial settings, there are many types of internet-connected physical equipment which may be exposed to cyber criminals. The rise of industrial attacks puts the safety of an entire population at economic and physical risk. In December 2015, the Ivano-Frankivsk region of Western Ukraine experienced a loss of power after cyber attackers remotely took control of the cursors of employees at 3 power stations, changed their passwords, shut off power to every region they managed, disabled backup power, reset employee passwords, leaving 230,000 residents without power [68]. Between 2005 - 2010, a worm was discovered on computers and industrial programmable logic controllers (PLC's) which manage Iran's nuclear program [31]. The join effort between the US and Israel degraded Iran's nuclear centrifuges was highly sophisticated, only targeted machines related to the nuclear program, faked sensor data on the machines, 
destroyed $1 / 5$ of its nuclear centrifuges by spinning them out of control, and went undetected for 5 years [31] [67]. Politically motivated attacks against public infrastructure pose an imminent economic and physical threat to all people.

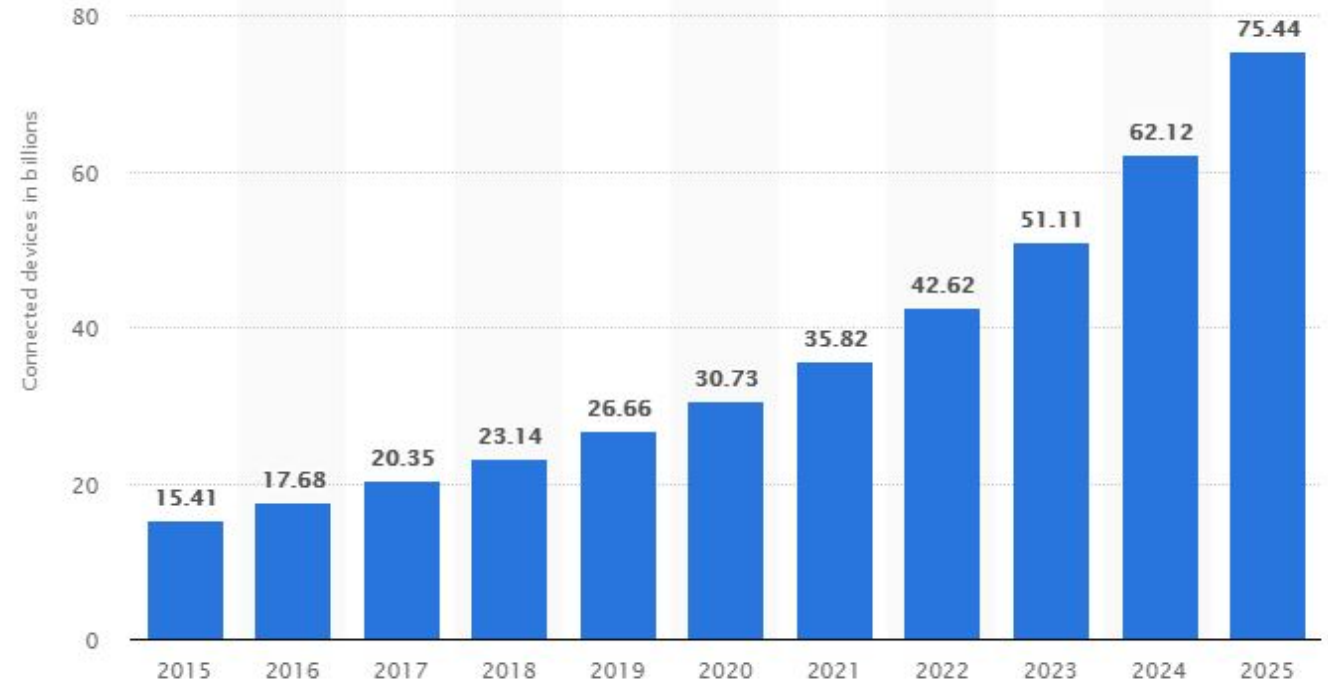

Figure 1.1: Projection of Worldwide Total M2M devices, created by Statista.

Increased internet connections and traffic will expose more machines to cyber attacks. Cisco explains 4 reasons that network usage are likely to become significantly larger in the near future: an increase in internet users, more machine-to-machine (M2M) devices, faster internet speeds, and increased video viewing. From 2016 - 2021 users are projected to increase from 3.3B - 4.6B, M2M devices will increase 17.1B 27.1B, average broadband will increase from 27.5 Mbps - 53.0 Mbps, and video viewing will increase from $73 \%$ - $82 \%$ of total traffic [21]. All of these advances expose more people and more machines to cyber threats. The increase in IoT (M2M) devices, as estimated by Statista [19], and shown in figure 1.1, is particularly concerning, because these are often unsecure. According to a 2017 study by the Ponemon Institute, 80\% 
of IoT applications are not tested for vulnerabilities [41]. This is understandable, as an influx of countless cheap IoT devices streaming from many manufacturers means that there is not much funding available for security testing, and that there is a litany of development platforms of varying security.

It is clear that Cyber crime is a growing and vastly underestimated threat. As the world puts its businesses, personal lives, personally identifiable information, and credit card and banking information online, cyber crime is more likely to affect everyone, not only businesses and governments. Given the growing prevalence of cyber attacks, it behooves all internet users to understand the large historical trends of previous cyber attacks, in the hope of predicting future cyber attack behavior.

Unfortunately, there is a dearth of open and organized knowledge about cyber attacks. The current data sources are discussed at length in chapter 2, Background. In this thesis, I assemble a database of cyber attacks 2014-2017 without paying for any data access, by using a semi-open data source. I then make various types of queries against the database to determine how cyber attack volume changes over time, what socioeconomic factors exacerbate cyber attacks, and what other unknown facets of cyber attacks can be discovered. The main results of these questions are as follows. From 2014-2017, global cyber attack volume peaks August 2016, and the United States reports the most cyber attacks received. Before August 2016, attacks against the private sector and governments dominated; after August 2016, attacks against individuals started to increase, and now are the most targeted victims. Political and economic cycles play an important role in cyber attack volume. Cyber attacks sometimes peak 3 months prior to a national election, while others peak directly following an election, as a means of protest. Wealthier countries receive more attacks, and in the case of Great Britain, cyber attacks usually peak in November each year. One interesting discovery is that, following the peak of cyber attacks in August 2016, by October 2016, cyber attacks were less than half of the peak, and by December 2016 
they approached levels similar to the maximum. Strangely, it seems that after a huge push for cyber attacks, hackers take a break for 2 months, subsequently returning to business as usual. 


\section{Chapter 2}

\section{BACKGROUND}

In this chapter I discuss previous works similar to this thesis, what they teach, and how my thesis stands apart from rest. Most previous work on global trends in cyber attacks does not suffice, as it falls into one of the following categories: it discusses only a subset of cyber attacks, is not statistically rigorous, or does not use an open data source. No previous work has generated a cyber attack database for public use as mine does.

In Debeck's, 2011 MS cybersecurity thesis, "The Correlates of Cyber Warfare: A Database for the Modern Era", he discusses the general ignorance and disorganization of data pertaining to cyber attacks, and proposes a worldwide network of routers to track attacks as they occur [37]. This hypothetical system would provide perfect knowledge of the country of origin of any attack, tracking the attack as it passes through various routers. Debeck's motivation for such a tremendous undertaking is the hope of correlating cyber attacks with political, cultural, and socioeconomic conditions in a way analogous to "The Correlates of War" project which does the same for natural war [1]. This work's emphasis on a general void of reliable and open cyber attack data, which holds true 7 years after its release, was the original impetus for this thesis. Unlike Debeck, in this thesis, the cyber attack database I create is not a hypothetical one, but actually created from cyber attack data.

Ghandi's 2011 IEEE article, "Dimensions of Cyber-Attacks: Culture, Social, Economic, and Political" identifies a few dozen cyber attacks that are directly linked to socioeconomic events [42]. By scouring news articles, and performing in depth research on the attacks and their motivations, Ghandi makes the case that socioeconomic events are a substantial motivator of cyber attacks, and that their crucial 
role in cyber attacks requires further research. However, Ghandi's does not statistically correlate any socioeconomic factors with cyber attack volume; it is more of a narrative that connects socioeconomic and political headlines with cyber security breaches. Ghandi states that, "the movements of Russian troops in Georgia were correlated with cyber-attacks on the Georgian communications infrastructure and defacement of government websites." This is believable given the evidence presented, but there is no mathematically derived correlation, which is generally a Pearson-R correlation. Again, Ghandi notes, "[a lack of cyber attacks] seems to reinforce the notion that attacks are strongly positively correlated to political and cultural conflicts", without deriving the actual correlation. In general, the work is believable, but of small scope, lacking in statistical rigor, and mostly anecdotal. In contrast, this thesis rigorously quantifies cyber attack volume for various countries, target sectors, types of attacks, and the various correlations between types of cyber attacks, as well with socioeconomic data.

Sharma's 2013 work, “A Social Dimensional Cyber Threat Model With Formal Concept Analysis and Fact-Proposition Inference", the authors describe how to use the socioeconomic conditions surrounding cyber attacks to server the construction of a decision tree that can characterize and predict cyber attacks using fact proposition inference [63]. In the fact proposition inference model used in this work, facts are inferred from propositions via a Bayesian belief network, where propositions are analogous to antecedents, and facts are analogous to consequents. Though Sharma focuses on the ontology of socially-motivated attacks, he claims tangible results from unnamed cyber attack data sources. For instance, the consequence "Information and Data Loss", the victims "Individuals/Civilians", the means, "Penetration Attempt", and the technological aspects, "SQL and Code Injection" all represent the highest beliefs in their category in the belief network. Unfortunately, the web app created for this paper is not available, the belief network is never shown in its entirety, and 
the cyber attacks data source is never revealed. All of these factors make the results difficult to use or rely upon. In contrast, in this thesis I reveal my data source, and open source my work for others to reproduce.

In her 2014 paper, "Global Mapping of Cyber Attacks" Carley does much of what I do in this thesis [33]. She quantifies types of attacks, identifies which countries receive the most cyber attacks, and correlates volume of cyber attacks received with various socioeconomic data. Carley's analysis is truly an impressive work, and it relies upon her previous work of simply creating a database of cyber attacks [56]. Unfortunately, Carley's work relies upon closed data. The attack signatures are freely available, but the number of Symantec machines who have experienced an attack, and the IP addresses of those machines, which makes identifying the nation of any particular machine affected possible, are all closed source. This was only possible because Carley and her fellow researchers were granted special access to Symantec's World Intelligence Network Environment (WINE) Intrusion Prevention System's (IPS) telemetry data. This real world data sampled from over 10 million machines around the world no doubt allows for the highest quality of data, but it is infeasible for most researchers. Carley also does not analyze temporal changes in attack data at all, and only analyzes attacks from November 2009 - September 2011. In contrast, I only use open data, I analyze temporal and seasonal trends in different types of cyber attacks and their victims, and I analyze cyber attacks from January 2014 - February 2018, a longer and more recent history.

Aviles' 2015 master's thesis in cybersecurity, "How US Political and Socio-economic Trends Promotes Hacktivist Activity" tries to understand hacktivism, among other things, by analyzing cyber attack reports from Hackmageddon [25] [60]. Unfortunately, the work is very anecdotal, and only examines cyber activity in December 2014 and January 2015. The only charts and results obtained are mere reproductions from Hackmageddon [60]. This work is not statistically rigorous, but a qualitative 
approach to seeing cyber attacks through a socioeconomic lens. I also use Aviles' data source. However, in this thesis I perform rigorous statistical analysis.

Kumar's 2016 research paper, "DDoS Cyber-Attacks Network: Who's Attacking Whom?", analyzes global trends in DDoS attack behavior [51]. DDoS, or Distributed Denial of Service, is a type of attack in which a machine is overwhelmed by the network requests made by thousands of machines in different geographical locations, causing the victim machine to become unavailable. Using open DDoS data from Digital Attack Map [6], Kumar discovers that, from May 2013 - March 2016, the top 10 victims of DDoS in descending order are the US, China, Peru, France, Canada, Poland, Great Britain, Brazil, Germany, and South Korea. The top 10 DDoS aggressors in descending order are China, the US, the Netherlands, Germany, South Korea, Brazil, Great Britain, Russia, France, and Turkey. Kumar notes that the country of the attacker is only known for about $\frac{1}{3}$ of the attacks. It is similar to what I achieve in examining international relationships with respect to cyber attacks. However, as this work only examines DDoS attacks, one of many types of attacks, it is more limited in scope, than my own. I examine all types of cyber attacks, and I analyze many other aspects of cyber attacks than the aggressor country and victim country.

Solano's 2017 IEEE conference paper, "Socio-economic Factors in Cybercrime" progresses Ghandi's work with much more statistical rigor, correlating cyber attack incidents with 32 socioeconomic factors, including GDP PPA, unemployment, political stability, freedom of press, happiness, access to broadband internet, population, and life expectancy [64]. The cyber attack incidents are derived mainly from reports from www.hackmageddon.com [60], and socioeconomic sources are derived from the World Bank [15], the International Labour Organization [10], Freedom House [8], Polity IV [54], Reporters Without Borders [14], Transparency International [13], Credendo Group [4], and The Economist Intelligence Unit [3]. Solano's work is one of the more complete studies on the socioeconomic correlates of cyber crime to date, 
but all results that are not extremely strong correlations are discarded. Solano believes that only 3 correlations, all greater than 0.80 and with p-values all less than 0.05, are important enough to discuss. Discussion of interpretation of Pearson correlations and p-values is in section 3.7. Solano notes 2 other statistically significant results with correlation greater than 0.60 disparagingly. This is a great disservice, as Pearson correlations greater than 0.50 are considered strongly correlated [36]. It is therefore very likely that Solano obtained many important results which will never see the light of day. Solano finds that in Syria, the correlation between political risk and the number of attacks received is $(r=0.864, p=0.012)$, in Mexico, the correlation between lending interest rates and attacks received is $(r=0.840, p=0.036)$, and that in Ghana, the correlation between GDP and attacks received is $(r=0.821$, $p=0.045)$. Additionally, the correlation between the perception of corruption of Russia and attacks on Australia to be $(r=0.642, p=0.025)$, and the correlation between the perception of corruption of Lithuania and Australia to be $(r=0.639)$, with a "similar" p-value. The authors of this paper are dissatisfied with the results, concluding, it "has not been able to find definite correlations between security incidents and the socio-economic variable of the countries involved", citing the under reporting of cyber attack incidents as a likely source of error [64]. By contrast, in this thesis, I reveal all of my results, including many in which there is no statistically significant correlation between cyber attack features. This is scientifically honest, and allows other researchers to replicate the results. 
Chapter 3

\section{THE CYBER ATTACK DATABASE IMPLEMENTATION}

To organize cyber attack data in a way that is structured, persistent, and easily accessible from a single endpoint, an RDBMS is selected. This SQL solution allows researchers around the world to apply data science to cyber attack trends. All other

options are inappropriate. Keeping data in spreadsheets or CSV's potentially splits data, does not allow complex queries, and does not enforce any rules for field values. Storage in document-oriented NOSQL databases such as Dynamo, Mongo, or Redis would not take advantage of any structure inherent in common data fields. A graph database such as Neo4j would allow for more complex connections made between attacks, but development time would be much longer, and because graph databases like Neo4j require learning a niche query language, the end result would not be accessible to the vast majority of cybersecurity researchers.

The implementation of a cyber attacks database is contingent on the available data. Finding reliable structured data on attacks can be very difficult, but we show how it can be done. Then the data is conditioned to fill in missing cells, and make text values more symmetric. Next, the data is transformed to a SQL-friendly version. Lastly, the SQL database is created and populated with the cleaned data.

\subsection{Open Data on Cyber Attacks}

Currently there are no viable open data sources of cyber attacks [37]. This makes sense because of the following. Private corporations study cyber attack data for sale, so they aren't motivated to maintain an open database of attacks. Government agencies also have their own private data stores on security incidents, but governments secu- 
rity agencies also want to conceal what they know, and could end up exposing their own state-sponsored attacks. So they are also motivated to keep data secret. Most individuals do not have the time or resources to maintain a database of cyber-attacks, which is constantly growing. Hence, most researchers don't have access to data regarding the trends in cyber attacks. Past research has required meticulously collected articles from security-oriented news sites, such as in Gandhi's "Dimensions of CyberAttacks"[42]. Other research, such as Kumar's "Cyber Attacks DDoS Network"[51], has relied on www.ddosmap.com as a data source for understanding DDoS attacks. Still other research, such as Solano's "Socio-economic factors in cybercrime" [64] and Avile's "How US Political and Socio-economic Trends Promotes Hacktivist Activity" [25], has used the data from www.hackmageddon.com to analyze larger cyber attack trends. This last option covers many different types of attacks (not only DDoS), is structured, contains enough metadata to make detailed analysis, is semi-open, and saves researchers the trouble of handpicking cyber attack incidents. Therefore, it is the sole cyber attack data source used in this thesis.

\subsection{Hackmageddon}

The site "Hackmageddon" is the labor of security professional Paolo Passeri [59]. Passeri manually collects the cyber attacks data from following many security news sites, and uploads graphs and charts of them which he uses in a security blog. Users of Hackmageddon can submit an attack, which Passeri will review, and may include in the data at his discretion. To gain access to the original data used on the security blog, this author needed to contact Passeri personally. Thankfully, Passeri freely opens the data to anyone who asks.

The data is in the form of Excel Spreadsheets, a file for each half of a month, with data from 2011 to present (March 2018). Excel is Passeri's primary way of storing and 
analyzing attacks. The fields are as follows: ID, Date, Author, Target, Description, Attack, Target Class, Attack Class, Country, Link, Tags.

1. ID: An integer unique to that half-month file

2. Date: The date the attack was reported

3. Attack: The type of attack (e.g. DDoS)

4. Target Class: The sector targeted.

5. Attack Class: Cyber War, Cyber Crime, Cyber Espionage, or Hacktivism

6. Country: ISO alpha-2 country code (e.g. US)

7. Link: A URL for a new article describing the attack

8. Tags: Important keywords

Often Author and Target are missing, and for older spreadsheets the Author field is actually a picture of the country, or hacker organization. For example, if Anonymous is behind an attack, a .png of a Guy Fawkes mask is often placed in the Author field.When the Author field is known, the picture must be replaced with the Author in plain text. Additionally, the Author and Target fields are sometimes empty. If the Author or Target is made known in the Description field, it must be filled. Otherwise, the field should have "NULL" as its value. Fields that are left empty, or filled with a "?" must all be filled with "NULL" as their values. Because a single spreadsheet holds only half of that month's cyber attacks, they must also be combined into a larger spreadsheet to make insights into the higher level attack trends. Filling in missing data and making field entries consistent requires considerable work, and inspecting every entry for an entire year, approximately 1000 entries, takes about a day. Examples of "Attack" include "DDoS" and "Account Hijacking". Passeri has 


\begin{tabular}{|c|c|}
\hline Field & Example \\
\hline Id & 16 \\
\hline Date & $17-10-2016$ \\
\hline Author & Guccifer2.0 \\
\hline Target & Democratic National Committee(DNC) \\
\hline Description & $\begin{array}{l}\text { Guccifer } 2.0 \text { is back and leaks new fresh documents } \\
\text { relating to the US political system (documents al- } \\
\text { legedly showing email conversations between DNC } \\
\text { employees and Hillary Clinton's presidential cam- } \\
\text { paign staff discussing Donald Trump's position on } \\
\text { his tax returns). }\end{array}$ \\
\hline Attack & Unknown \\
\hline Target Class & Org:Political Party \\
\hline Attack Class & $\mathrm{CC}$ \\
\hline Country & US \\
\hline Link & $\begin{array}{l}\text { http://www.ibtimes.co.uk/hacker-guccifer-2- } \\
\text { 0-leaks-files-claiming-dnc-researched-donald- } \\
\text { trumps-taxes-1587073 }\end{array}$ \\
\hline Tags & $\begin{array}{l}\text { Guccifer 2.0, Democratic National Committee, } \\
\text { DNC, Hillary Clinton, Donald Trump }\end{array}$ \\
\hline
\end{tabular}

Table 3.1: The fields of the "attacks" spreadsheets used by Hackmageddon, and a sample row.

defined 4 abbreviations for Attack Class: CC (Cyber Crime), CE (Cyber Espionage), CW (Cyber War), and H (Hackitivism). Sometimes this field has the full name of the Attack Class, and sometimes many attack classes are listed. The "Country" field refers to the victim country. This is easily determined in instances of cyber war, or 
when a large group of consumer that mostly or solely live in a certain country are targeted, though some attacks target a few or many countries. In these cases, the "Country" either lists the ISO alpha-2 country codes of 2-3 countries delimited by spaces or commas, or may simply read, "> 1".

\subsection{Making the Spreadsheets SQL-Friendly}

Using the raw attack data contained in separate spreadsheets proves unwieldy to deep data analysis. This problem is not assuaged even after they compiled into larger records. So, they are entered into a MySQL database. There are some issues that must be rectified first. The "Id" field is only unique across one spreadsheet (half a month of attacks), and must be unique across the entire database. The "Date" field is a reserved word in SQL so it is changed to "AttackDate". The entries are in the European format, "d/m/yyyy", instead of the standard SQL format, "yyyy-mm-dd". The "Target Class" and "Attack Class" fields contain spaces. So these all need to be changed to "TargetClass" and "AttackClass". "Country" is also ambiguous, as a user may interpret this as the author's country or the victims' country. To remove the ambiguity, this is changed to "TargetCountry". Another consideration is that though the "TargetCountry" field may reference another table of country data, sometimes Passeri's attack entries have more than one country per entry. Therefore, the "TargetCountry" field cannot be queried by a simple "GROUP BY" query, and cannot be a foreign key to a countries database. However, this is not a major problem, because only a few countries experience the majority of attacks, and general SQL commands are sufficient for seeing major attack trends and correlations. These insights can be used for more targeted SQL queries that get all attacks where "TargetCountry" contains "US", such as "US, UK". The last step is to convert the Excel spreadsheet to a comma-separated value (CSV) file in UTF-8 format. After these changes are made, 
the fields in Passeri's spreadsheets map directly to a MySQL schema, which shall now be described.

\subsection{Creating the Cyber Attack Database}

After all addressing all the necessary changes to the schema, the creation of the table is as follows:

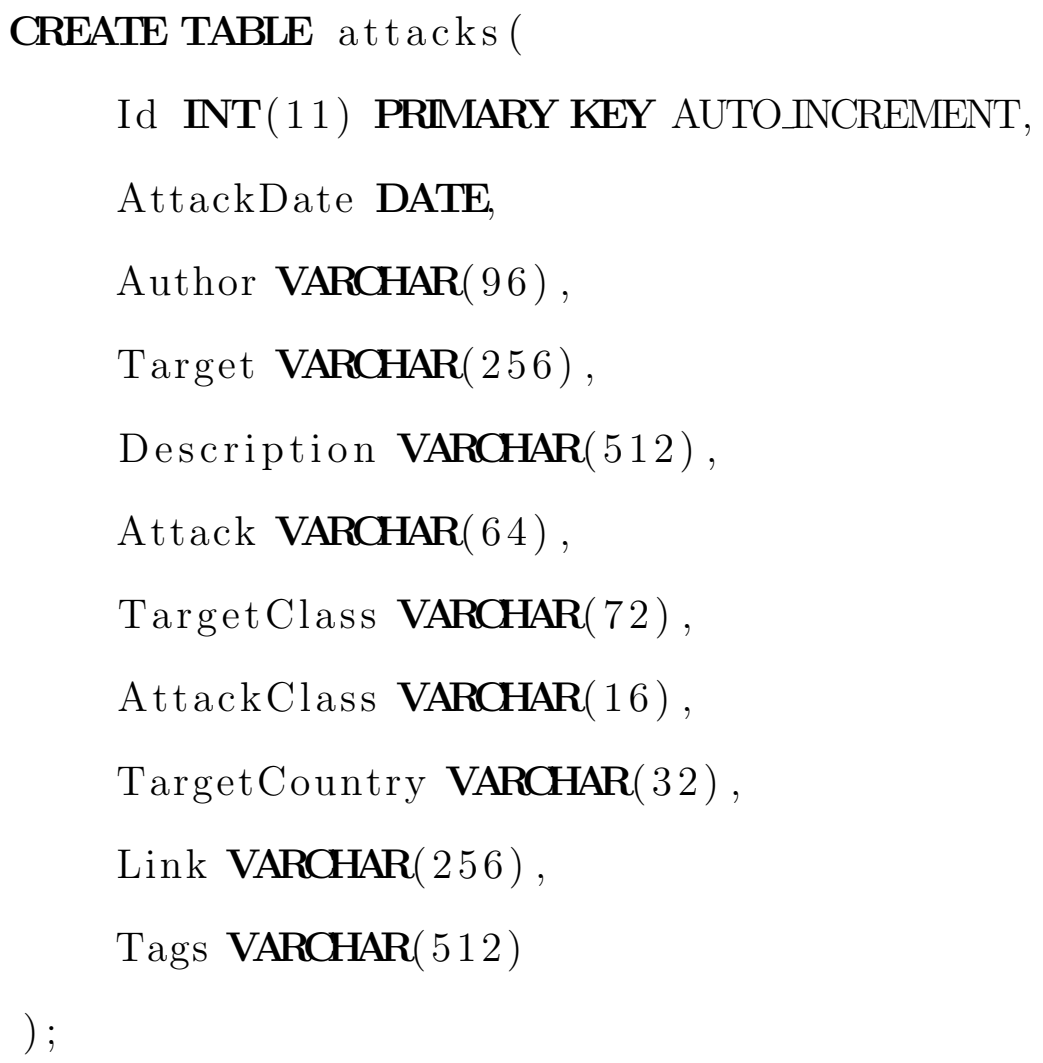

The table 3.2 shows the schema and a sample row.

\subsection{Inserting The Data}

After creating the database, it is populated with the sanitized data from the spreadsheets. The dates are reformatted to MySQL syntax form, then converted to UTF-8 encoded CSV's. The CSV's are combined into a single file representing a single year of 


\begin{tabular}{|c|c|c|c|}
\hline Field & Type & Size & Example \\
\hline Id & INT(11) & 4 & 38820 \\
\hline AttackDate & DATE & 3 & 2016-10-17 \\
\hline Author & VARCHAR & 96 & Guccifer2.0 \\
\hline Target & VARCHAR & 256 & Democratic National Committee(DNC) \\
\hline Description & VARCHAR & 512 & $\begin{array}{l}\text { Guccifer } 2.0 \text { is back and leaks new fresh } \\
\text { documents relating to the US political sys- } \\
\text { tem (documents allegedly showing email } \\
\text { conversations between DNC employees } \\
\text { and Hillary Clinton's presidential cam- } \\
\text { paign staff discussing Donald Trump's po- } \\
\text { sition on his tax returns). }\end{array}$ \\
\hline Attack & VARCHAR & 64 & Unknown \\
\hline TargetClass & VARCHAR & 72 & Org:Political Party \\
\hline AttackClass & VARCHAR & 16 & $\mathrm{CC}$ \\
\hline TargetCountry & VARCHAR & 32 & US \\
\hline Link & VARCHAR & 256 & $\begin{array}{l}\text { http://www.ibtimes.co.uk/hacker- } \\
\text { guccifer-2-0-leaks-files-claiming-dnc- } \\
\text { researched-donald-trumps-taxes-1587073 }\end{array}$ \\
\hline Tags & VARCHAR & 512 & $\begin{array}{l}\text { Guccifer 2.0, Democratic National Com- } \\
\text { mittee, DNC, Hillary Clinton, Donald } \\
\text { Trump }\end{array}$ \\
\hline
\end{tabular}

Table 3.2: The schema of the "attacks" table in the Cyber Attacks database, and a sample row. The "Id" field, an auto-incremented integer, is unusually large solely from adding and removing many entries to the table in development. 
attacks (again, about 1000/yr). Instead of reading the CSV's directly into a MySQL database, it is read into a "dataframe" object, a data type from the "pandas" python package, for inspection. This lets us double-check that all fields and rows look as they should, before adding them to the table. Pandas also does a nice job of handling null values without an explicit NULL in the cell, as well as escaping quotes and other sequences that are meant to be part of the field entry. After they are inspected, pandas dataframe objects have a nice to_sql() method we use for inserting data into the database an entire dataframe at a time.

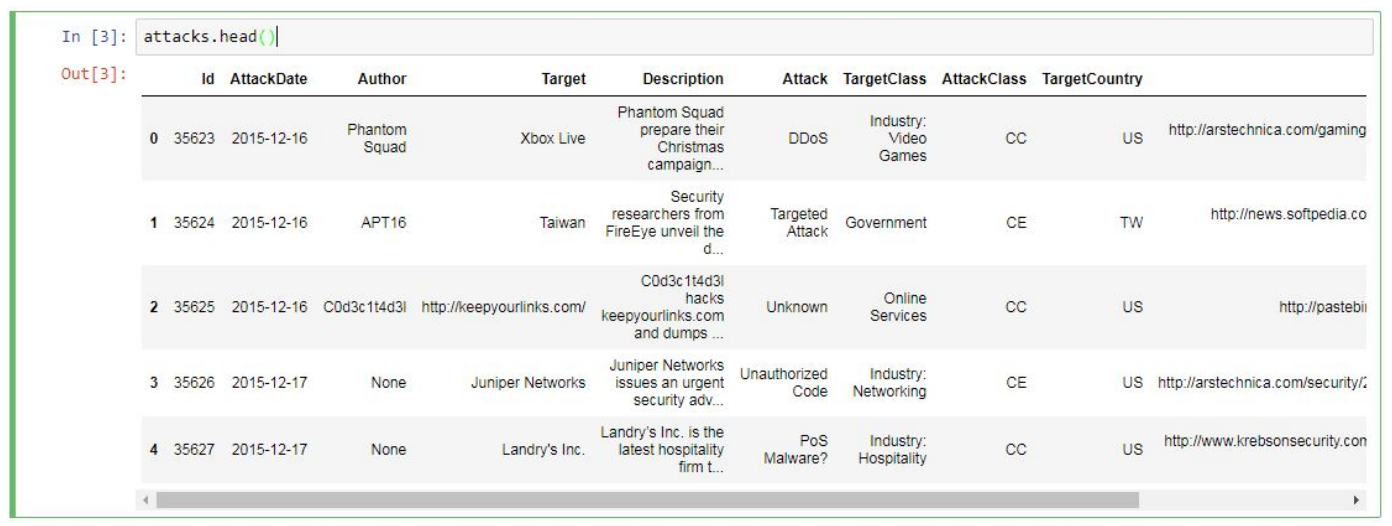

Figure 3.1: Inspecting the attacks as a pandas dataframe in a Jupyter notebook.

\subsection{Querying The Data}

SELECT TargetCountry, COUNT(*) as num_attacks

FROM attacks

GROUP BY TargetCountry

ORDER BY num_attacks DESC;

The most interesting parts of the data are the volume of attacks per month, and how attack volume is correlated in various ways. We can get a quick look at attack volume by simple queries, such as the following one quantifying attack volume by country. 
This produces table 3.3. Often, there are discrepancies in how the TargetCountry or Sector is recorded, so a more flexible query can gather any attack in which 'US' appears, which may be in a field such as "US UK". So actual volume numbers are more accurately calculated using a query of the following form:

\section{SELECT COUNT $(*)$ as num_attacks}

FROM attacks

\section{WHERE TargetCountry LIKE "\%US\%";}

Doing this repeatedly for the top 12 countries in attack volume produces a more accurate estimate of attack volume. If make "countries" table, we have an easier way to get more reliable estimates for all countries of the form:

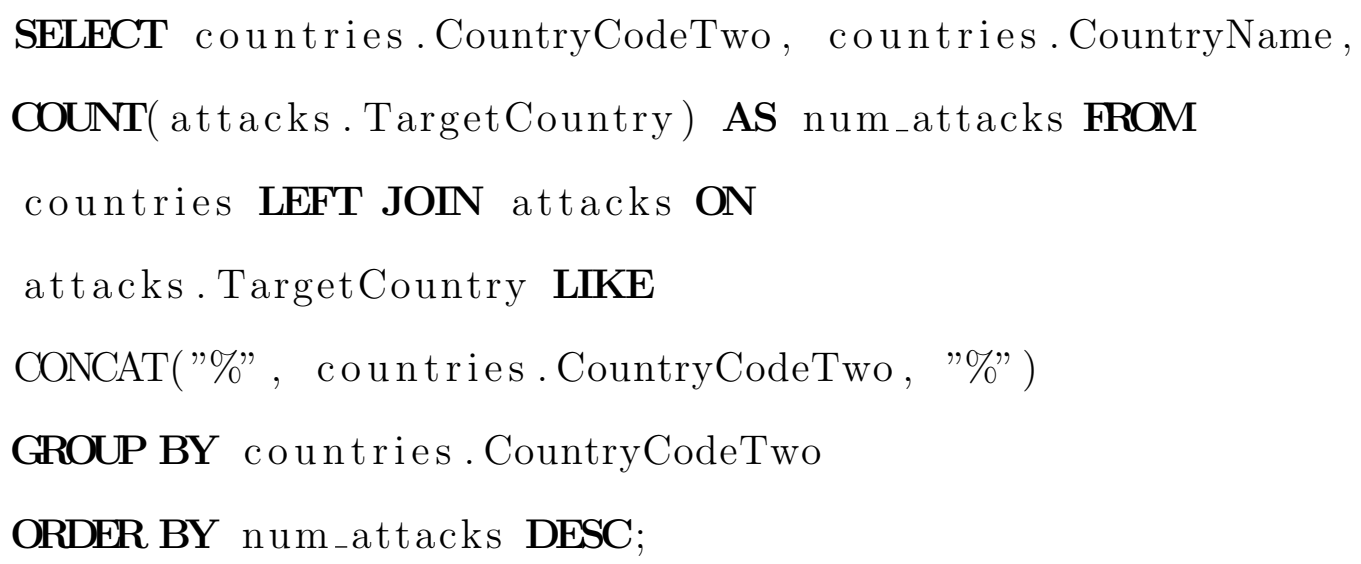

After getting an idea of the countries and sectors that are targeted the most, we can focus on detailed queries to gain insight into cyber attack trends.

\subsection{Analysis Overview}

The analysis is performed on all attacks recorded by Hackmageddon over the 4 year period from January 1, 2014 to January 1, 2018. Attack volume by country and sector are visualized for the countries and sectors that receive the most attacks. The correlation between attack volume for different sectors and different countries are analyzed by calculating the Pearson $r$ value and p-value. Recall that statistically 
significant p-values are less than 0.05 and highly significant values are less than 0.01 [40]. Also, recall the following about Pearson Correlation, in table 3.4. This shall become useful when analyzing the significance of our results. 


\begin{tabular}{|c|c|}
\hline TargetCountry & num_attacks \\
\hline US & 1693 \\
\hline$>1$ & 599 \\
\hline GB & 254 \\
\hline IN & 124 \\
\hline NULL & 110 \\
\hline $\mathrm{CA}$ & 96 \\
\hline $\mathrm{AU}$ & 77 \\
\hline RU & 71 \\
\hline IL & 65 \\
\hline FR & 58 \\
\hline $\mathrm{KR}$ & 57 \\
\hline UA & 55 \\
\hline$J P$ & 54 \\
\hline $\mathrm{CN}$ & 47 \\
\hline IT & 45 \\
\hline $\mathrm{DE}$ & 43 \\
\hline PK & 40 \\
\hline $\mathrm{BR}$ & 37 \\
\hline $\mathrm{TR}$ & 37 \\
\hline NL & 27 \\
\hline SA & 25 \\
\hline $\mathrm{PH}$ & 22 \\
\hline
\end{tabular}

Table 3.3: The top 20 countries by attack volume 2014-2017 inclusive, as queried on the attacks database using a more targeted query for $>1$ and NULL values, and the remainder of countries using the aforementioned LEFT JOIN technique. 


\begin{tabular}{|c|c|}
\hline Pearson Correlation & Correlation Interpetted As \\
\hline $0.00-0.10$ & very weak \\
\hline $0.10-0.30$ & weak \\
\hline $0.30-0.50$ & moderate \\
\hline $0.50-1.00$ & strong \\
\hline
\end{tabular}

Table 3.4: The interpretation of Pearson Correlation values [36]. 
Chapter 4

\section{CAPTURING SOCIO-ECONOMIC DATA}

As discussed, previous works have shown a correlation between socio-economic factors and the volume of cyber-attacks a country receives [64] [42] [25] [37]. Here, we aggregate many socio-economic factors from open sources to study the degree to which various socio-economic factors influence a country's volume of attack received. Most of factors are taken from Heliwell's World Happiness Report 2015 [49]. The data included on this web page in the form of Excel spreadsheets and found by clicking, "Chapter 2 Online Data Expanded with Trust and Governance" contain data on many socio-economic factors, which shall be detailed in the next section. However, because GDP was not included in this dataset, only a GDP per capita, whose precision had been destroyed by dividing by a very large number (population), additional GDP data is found through World Bank [26]. Two more fields "HappinessRank and "HappinessScore" are found through a dataset that Sustainable Development Solutions Network hosts on Kaggle [58]. SDSN is an organization which actively supports the World Happiness Report [9]. We shall now delve into the contents of these datasets.

\subsection{The World Happiness Report}

The first World Happiness Report was released in April 2012, and has released one report annually ever since, with its most recent report being released March 2018 [7]. Here we run 2 analyses: first of which only uses the 2014 data from the 2015 report, and the second of which draws from the aforementioned 2018 report, using only the data pertaining to years 2014-2017 (inclusive). All data collected in the World Happiness Report is taken from the World Gallup Poll [9]. 
Like the Hackmageddon data, before using the World Happiness Report data, it all needs to be wrangled [55]. In the first analysis, we are relating the socio-economic data from 2014 to the total attack volume over the total 2014-2017 period. This type of analysis assumes that the socio-economic factors relevant to a particular country don't vary significantly enough to account for the disparity in overall cyber attack volume. Conversely, in the second analysis, which compares total cyber attack volume by year to socio-economic data for that year, assays to relate changes in both year-toyear. The assumption is that using data from every year could produce more detailed results, but relating them to the only first year suffices for seeing large trends in how total attacks received in this 4 year period.

Next, because there are not any columns for ISO alpha-2 or ISO alpha-3 country codes, only full country names, they must be added. Without them, joining to the attacks table is error-prone. Then, two-word column names are combined into singleword names, and all missing values are filled with "NULL". Many columns are mostly empty as well, so these areas where data was too difficult to collect are discarded. Finally, we are left with the following fields.

1. CountryName

2. CountryCodeTwo

3. CountryCodeThree

4. Population

5. Region

6. HappinessRank

7. HappinessScore

8. LowerConfInt_Hap 
9. UpperConfInt_Hap

10. StudyYear

11. LogGDPperCapita

12. SocialSupport

13. HealthyLifeExpectatBirth

14. FreedomLifeChoices

15. Generosity

16. PerceptionOfCorruption

17. PositiveAffect

18. NegativeAffect

19. ConfidenceInGov

20. DemocraticQuality

21. DeliveryQuality

22. PeopleCanBeTrusted

The fields "HappinessRank", "HappinessScore", "LowerConfInt_Hap", and "UppererConfInt_Hap", do not appear in the original data from the World Happiness Report, though "HappinessScore" is simply "LifeLadder" in the original study as outlined in the WHR appendix [22]. Instead, the auxiliary columns are pulled from the Kaggle dataset [58] which derived from the original WHR dataset and its annual summary by a community of data scientists. Additionally, the happiness fields only pertain to the first inquiry, and in this first overall inquiry, only data from 2015 is used, 
because there aren't any datasets with happiness scores from 2014 or prior. Because socio-economic data does not vary much between years, but varies greatly between countries, 2014, the earliest year in the cyber attacks database is chosen. The implication is that socioeconomic conditions at the beginning of the study serve as an input which affects the output, cyber attack volume, over the subsequent years. To demonstrate the effects of this slight variability of socioeconomic data, consider how little the correlation between annual GDP and the total cyber attacks experienced 2014-2017 varies in table 4.1 There are not socio-economic data for 2014 for every country, in

\begin{tabular}{|c|c|c|}
\hline Socio-Economic Factor & $r$ & $\mathrm{p}$-value \\
\hline GDP2016 & 0.186 & 0.023 \\
\hline GDP2015 & 0.181 & 0.026 \\
\hline GDP2014 & 0.179 & 0.027 \\
\hline GDP2013 & 0.176 & 0.029 \\
\hline GDP2012 & 0.176 & 0.03 \\
\hline GDP2010 & 0.175 & 0.03 \\
\hline GDP2011 & 0.172 & 0.033 \\
\hline
\end{tabular}

Table 4.1: Annual GDP Correlates of Total Cyber Attack Volume Received 2014-2017 in ascending order by p-value.

which case, the most recent data is used. 112 countries are analyzed using 2014 data, 29 countries are analyzed using 2013 data, 8 are examined using 2012 data, 5 using 2011 data, 1 using 2007, and 1 using 2006 data. Most of the countries lacking data from 2014 are countries which also don't receive many cyber attacks. Though most fields are self-explanatory, a few are elusive. The "...ConfInt_Hap” fields are the lower and upper confidence interval of the happiness score, "HealthyLifeExpectatBirth" was originally "Healthy Life Expectancy at Birth", "FreedomLifeChoices" in its original form was "Freedom to Make Life Choices". The field "DeliveryQuality" is a weighted 
average of several fields. The Statistical Appendix for Chapter 2 of the 2015 World Happiness Report explains its significances as follows:

Variables in the expanded data set: Democratic and delivery quality measures of governance are based on Worldwide Governance Indicators (WGI) project (Kaufmann, Kraay and Mastruzzi). The original data have six dimensions: Voice and Accountability, Political Stability and Absence of Violence, Government Effectiveness, Regulatory Quality, Rule of Law, Control of Corruption. The indicators are on a scale roughly with mean zero and a standard deviation of 1 . We reduce the number of dimensions to two using the simple average of the first two measures as an indicator of democratic quality, and the simple average of the other four measures as an indicator of delivery quality, following Helliwell and Huang (2008) $[17]$.

Detailed information about all fields is also available in the same appendix for the WHR [17]. 


\section{Chapter 5}

\section{RESULTS}

Risk is often defined in the utility function:

$$
R=\sum_{k=1}^{N} P_{r_{k}} U\left(X_{k}\right) .
$$

where $P_{r_{k}}$ is the probability of an event occurring, in the kth state, and $U\left(X_{k}\right)$ is the utility (loss) function of resource $X$ in state $k$ [52]. Put simply, risk in any particular state is the probability of adverse event occurring multiplied by the adversity of the event. In the case of cyber attacks, the utility function often requires information such as money lost, loss of public trust, number of devices affected, logins stolen, etc. These are sometimes difficult to come by, and are not included in the Hackmageddon data. However, the probability that an attack will occur against a particular victim country or sector could be estimated by measuring the volume of cyber attacks against a certain country or sector. In this section I discuss the results of analyzing cyber attack volume. Grouping attack victims by target country and target class (sector) allows us to identify the most targeted countries and sectors at any granularity of per diem or greater. This information is valuable because countries and sectors that are targeted at high frequency can generally expect to be targeted at high frequency in the future. In other words, a kid who is frequently bullied is at greater risk of future bullying than a kid who is not. Therefore, by measuring the volume of cyber attacks that victim countries and sectors receive, I am actually measuring the expected value of cyber attack volume for a country or sector, which as previously shown, is part of the risk equation. We measure the probability by taking advatage of the fields "TargetCountry" and "TargetSector", using them to make queries of the form, 


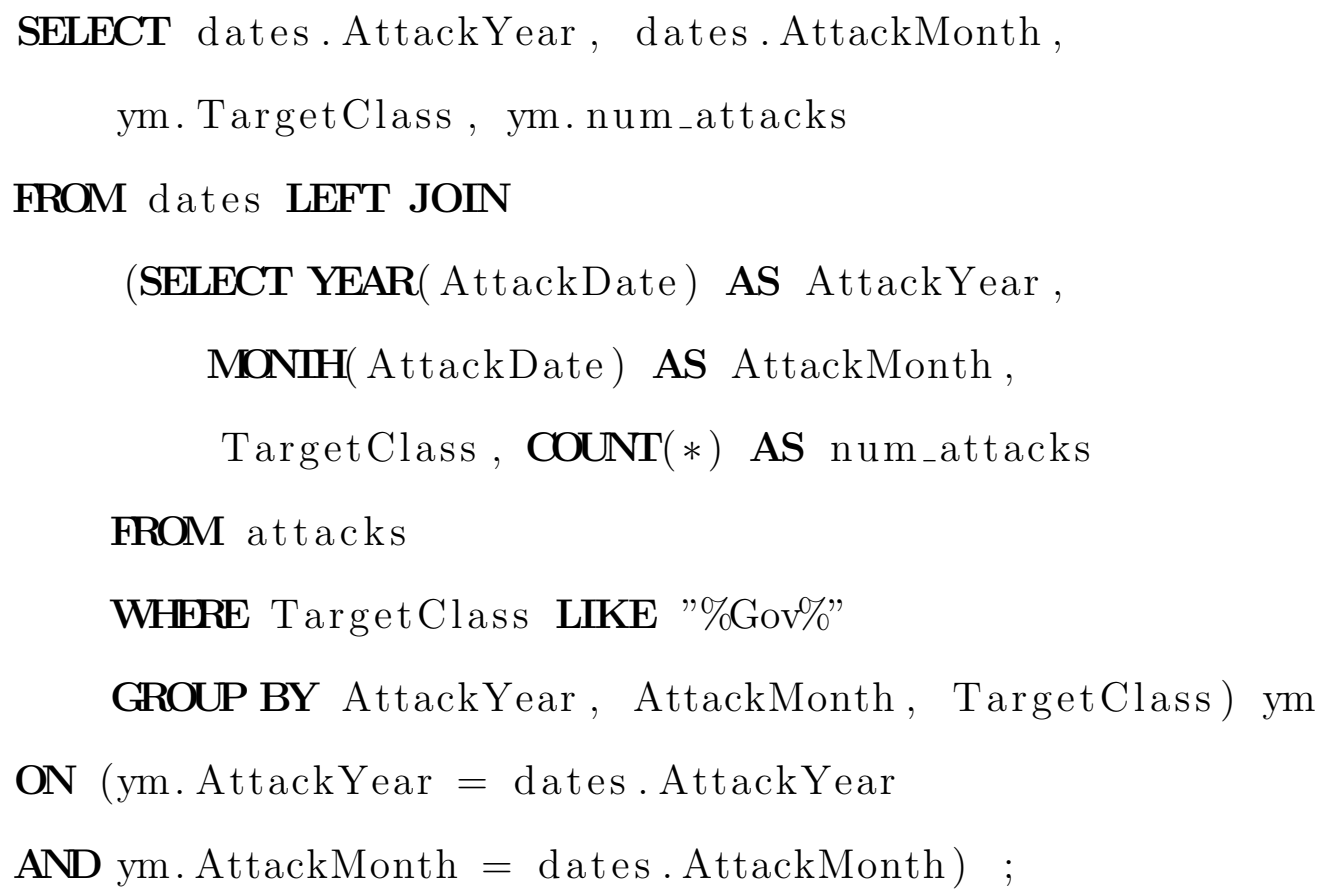

This chapter is divided into By Country, By Sector, and Socio-economic Factors. In the section By Country 5.1 I give an overall understanding to how attack volume varies temporally and across target countries, and expound on which country pairs' attack volume is most closely correlated. Because these two studies don't share much overlap, I divide them into two distinct sections. The first answers the first question of this thesis, "How does cyber attack volume vary with time?" for various countries. The second answers the third question, "What else can we learn about trends in cyber attacks?" I perform the data analysis on temporal data, and visualize it via simple graphs displaying the volume of cyber attacks, as well as Pearson $\mathrm{R}$ correlations. In the Sectors section I perform the same analysis on the most targeted sectors. Here there is considerable overlap between the most targeted sectors and the most correlated sector pairs (by attack volume received), so they are addressed in a single section. I examine cyber attack volume against certain countries and sectors all on a month-by-month basis. This is a large enough window that attack volume against minority targets and sectors is nontrivial, but granular enough to answer the first 
question of this thesis. The section "Socio-economic Factors" only covers correlates, because the census data for these factors are only taken once a year, whereas cyber attack traffic is analyzed at the smallest level on a month-by-month basis. This lays the groundwork for understanding the likelihood of attack, one of the components of risk assessment. The overarching implications of these results are covered in the Discussion section.

\subsection{By Country}

Countries that receive the most attacks are often not correlated with one another, but are of the most importance. Because their is not much overlap between these two groups, it is useful to break the study into two sections:

1. Trends 5.1.1: Study the cyber attack volume per month of the most heavily targeted countries in a subsection of their own

2. Correlates 5.1.2: Study the country pairs whose attack volumes are most closely correlated

\subsubsection{Trends}

In this section I discuss the results of analyses on temporal data, and their clustering by month, on the most targeted countries. Clustering by month is easy to do in SQL, and also is very easily understood by people. Clustering smaller, on a week-by-week basis is more difficult to visualize over the 4 year period, and loses some meaning. Clustering on a larger basis, such as by year, obscures how cyber attack volume varies wildly throughout the year. In figures 5.1 - 5.16 I show the cyber attack volume per country. By analyzing and visualizing this data we can better understand how the volume of cyber attacks changes over time for each country. Linear trends, or cyclical 
patterns may appear, which are easier to understand visually.

In figure 5.1 I show the total attack volume between January 2014 and February 2018. Note that figures 5.1 and 5.2 represent a high attack volume relative to the change in volume, so they are not shown with a y-axis starting at zero. In figure 5.1 the graph is jagged, with a global maximum of 116 attacks in August 2016 followed by a global minimum of 50 attacks two months later. This jaggedness means that cyber attack volume is changing dramatically in irregular ways. It may indicate dramatic changes in socioeconomic conditions. In figure 5.1, I plot the average of total attacks over the past six months in order to produce a smoother line that helps interpret larger trends. We see in figure 5.1 that rolling average total attacks generally increases from the start of the rolling average plot in June 2014 until the global maximum in August 2016, dipping until July 2017, and ramping back up until at least January 2018.

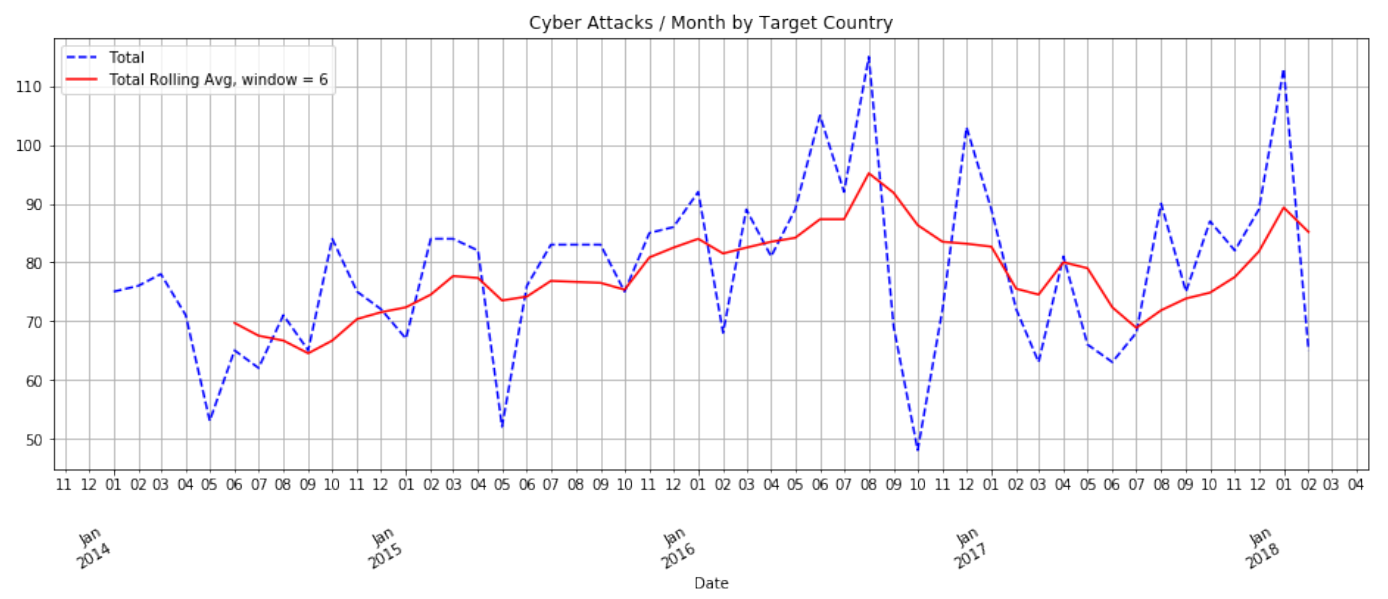

\section{Figure 5.1: The timeline of total cyber attack volume, with a 6 month rolling average.}

The graph in figure 5.2 is strikingly similar to the previous. 5.1. This is due in large part to the fact that the US receives more attacks than any other country recorded in this database. As previously shown in 3.3 the US receives more than 6.5 times the cyber attacks as the next most targeted country, Great Britain. As I illustrate in figure 5.3, the US dwarfs the next 4 most targeted countries by an order 


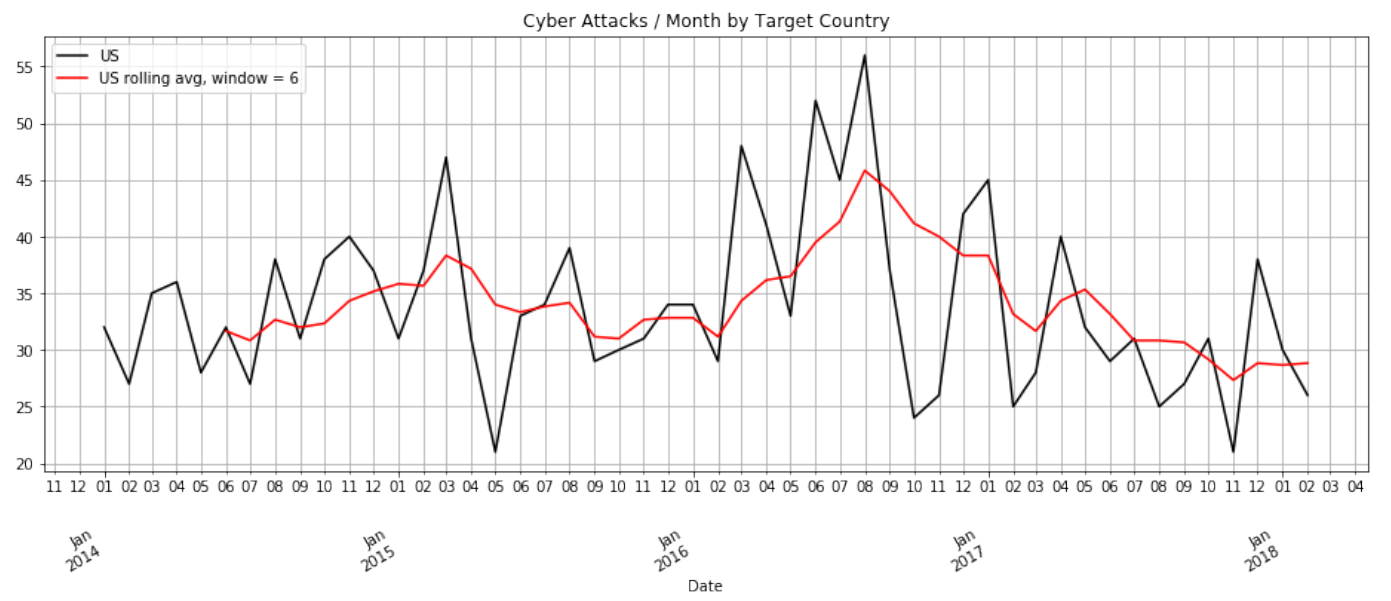

Figure 5.2: The timeline of US attack volume for the US with a 6 month rolling average.

of magnitude.

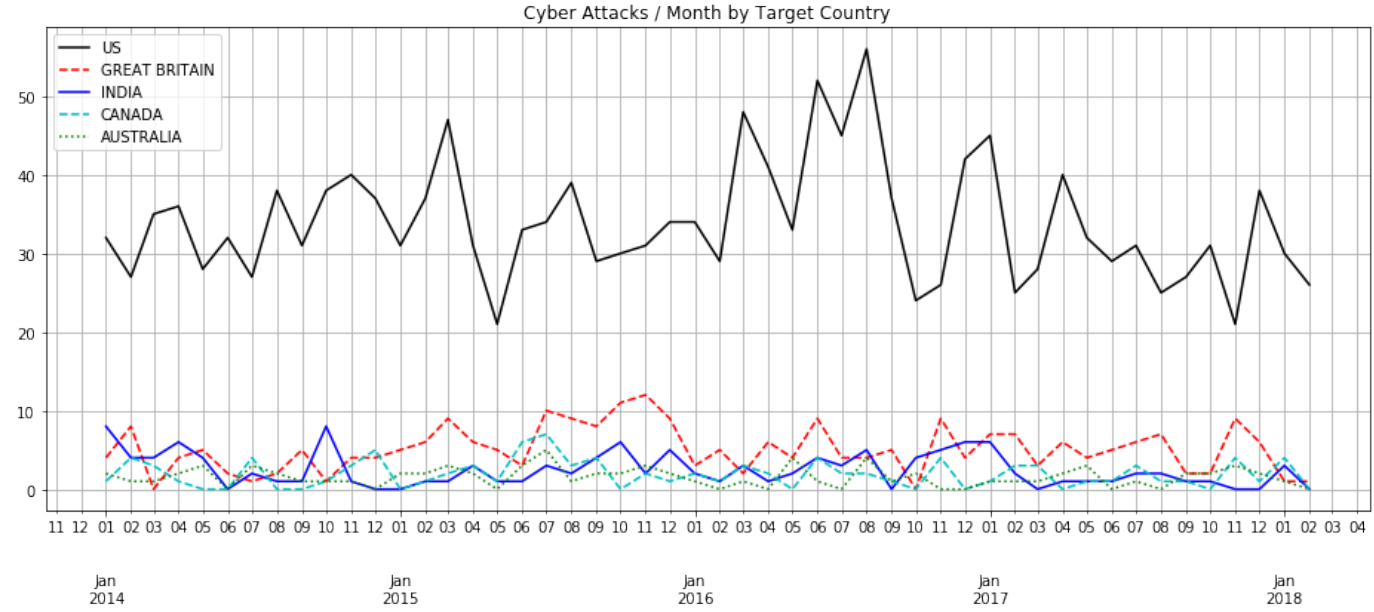

Figure 5.3: Attacks by Country Timeline - Timeline of cyber attack volume by country for top 5 victims.

Great Britain and India are $2^{\text {nd }}$ and $3^{\text {rd }}$ most attacked countries, respectively. Their graph in figure 5.4 confirm visually what is discovered through their Pearson correlation: they aren't correlated in any significant way $(r=0.248, p=0.082)$. All Pearson correlation values are on a volume-per-month basis, and can be found in table 5.1. Great Britain has a global maximum of 12 attacks in November of 2015. Great 
Britain has global minima at March 2014 and October 2016. India's attacks peak at global maxima 8 in Jan 2014 and Oct 2014, with global minima of zero attacks recorded at June 2014, Dec 2014, Jan 2015, Sept 2016, March 2017, Nov 2017, Dec 2017, and Feb 2018.

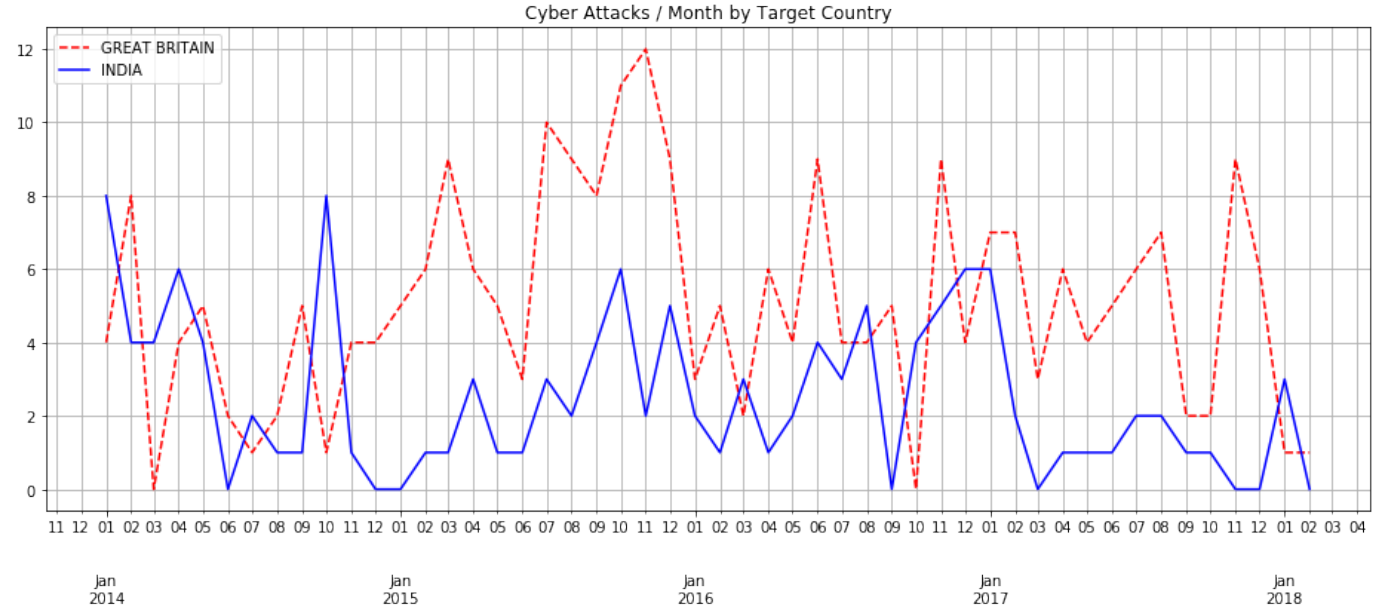

Figure 5.4: Timeline of cyber attack volume by country for Great Britain and India

By volume of cyber attacks received, Canada and Australia occupy spots 4 and 5 respectively. They are also not correlated in any significant way $(r=0.156, p=$ 0.279). Canada peaks in July 2015, with 12 months of 50 receiving zero reported attacks. Australia peaks in May 2015 and August 2015, with 10 months receiving zero reported cyber attacks.

The cyber attacks reported for the 6 and 7 spots, Russia and Israel, also uncorrelated $(r=0.086, p=0.552)$, are increasingly sparse as shown in figure 5.6. Russia has 13 months with zero attacks recorded, and Israel has 21 such months. There doesn't seem to be much here. With few attacks, there doesn't seem to be much we can learn. In the next subsection 5.1.2, we will see that that is not necessarily the case.

In figure 5.7 I show the average cyber attacks per month for the US. The maximum 


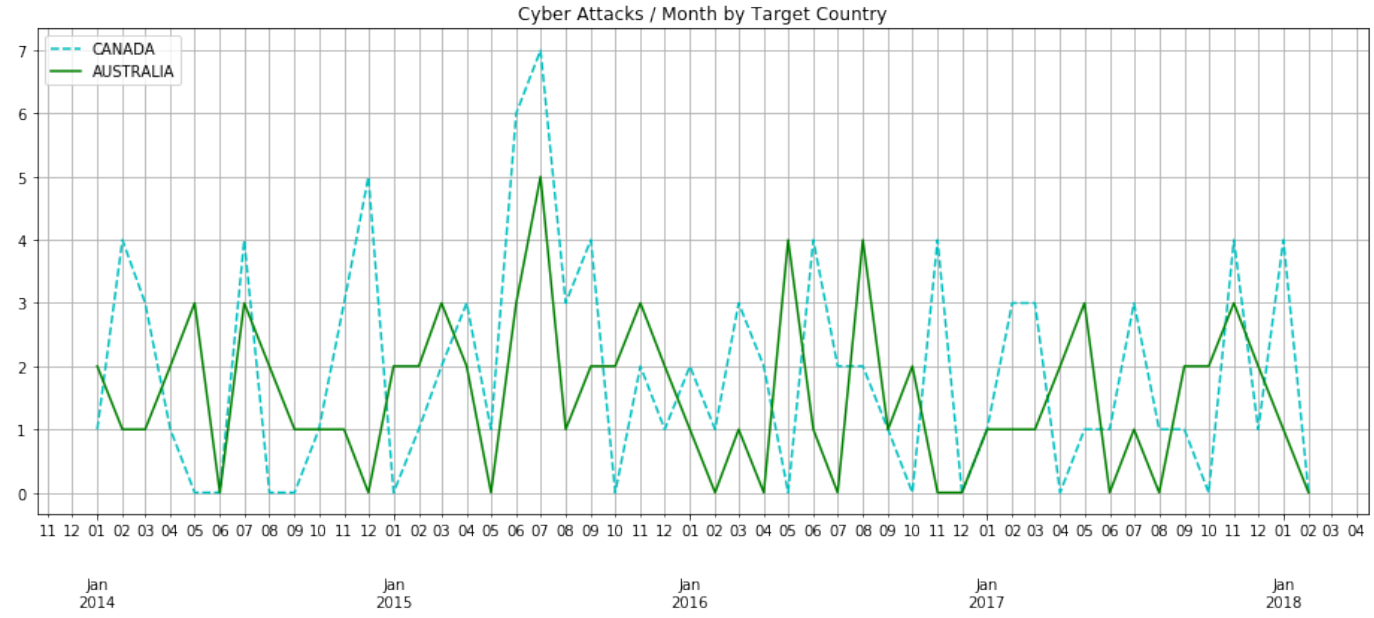

Figure 5.5: Timeline of cyber attack volume by country for Canada and Australia

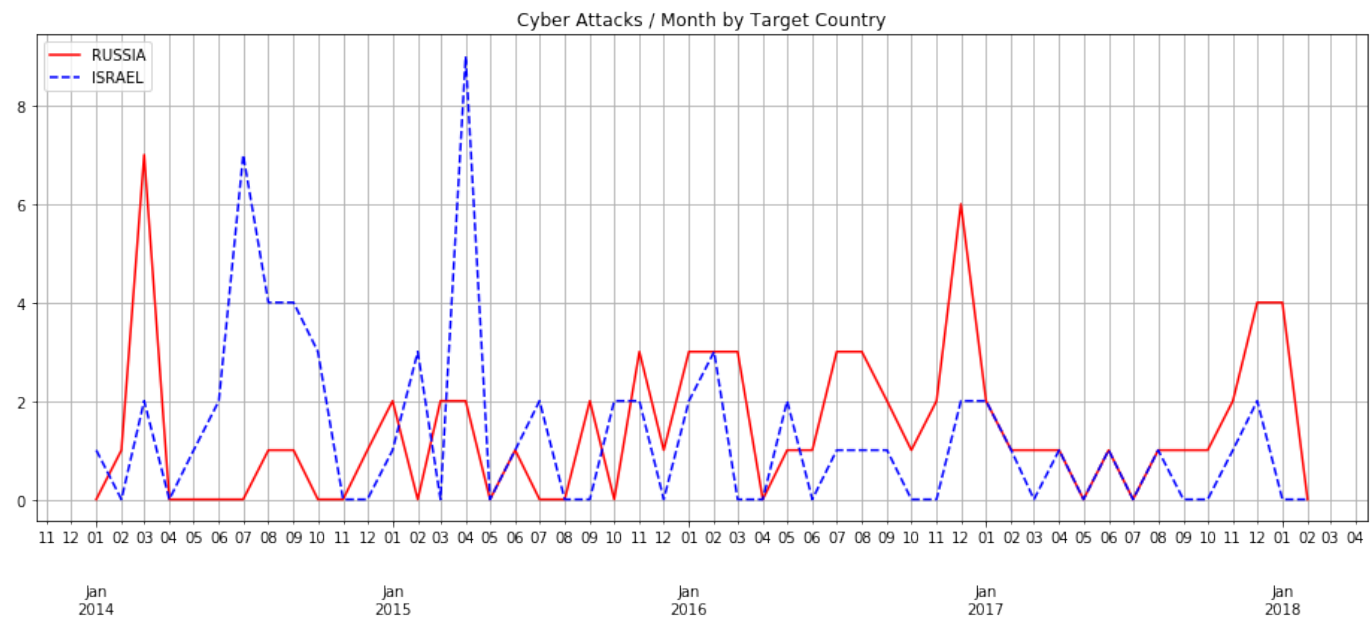

Figure 5.6: Timeline of cyber attack volume by country for Russia and Israel

average attacks per month also lands on the global maximum over the four year period, in August. A quick glance back at figure 5.2 shows that August was not the maximum for years 2014, 2015, or 2017. Though August was the third highest month in 2014, the second highest month in 2015, it was the second lowest in 2017. The massive spike in 2016 therefore played a significant role in making August the highest month on average for the US. 


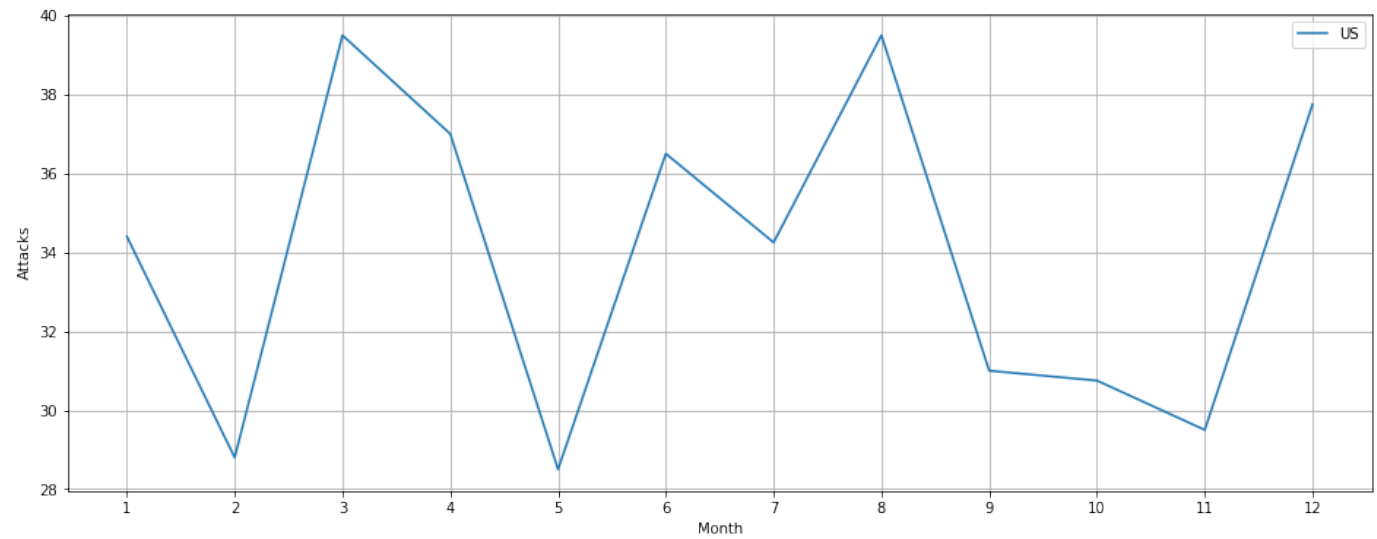

Figure 5.7: Average Cyber Attacks / Month for the US.

In figure 5.8 it can be seen that Great Britain's cyber average cyber attacks peak in November. Glancing back at 5.4 it is shown that in 3 out of 4 years, Britain's cyber attack volume peaks in November.

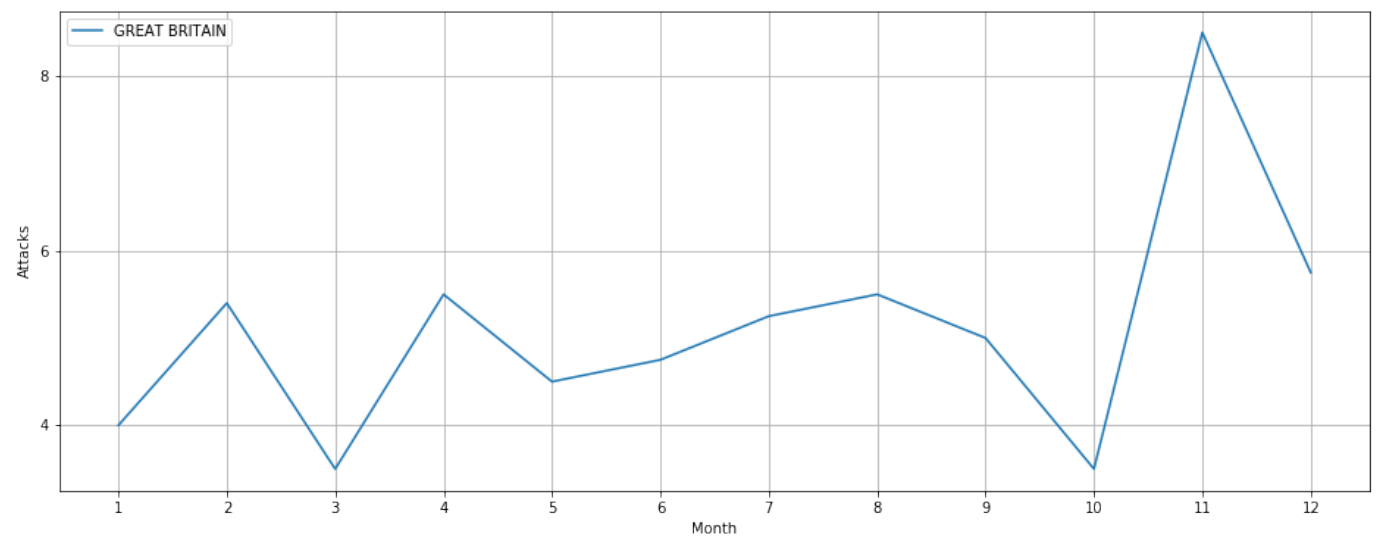

Figure 5.8: Average Cyber Attacks / Month for Great Britain.

India's attack volume hits a global maximum on average in October, as seen in fig 5.9. This behavior is expected, as India's cyber attack volume peaked in both October in 2014 and 2015, and October is the fourth most targeted month for India in 2016 and the fifth most targeted month for India in 2017.

Pakistan's by month average is shown in 5.9. Because Pakistan is the fifteenth most attacked nation, at only a sum of 40 attacks over the 4 year period, small 


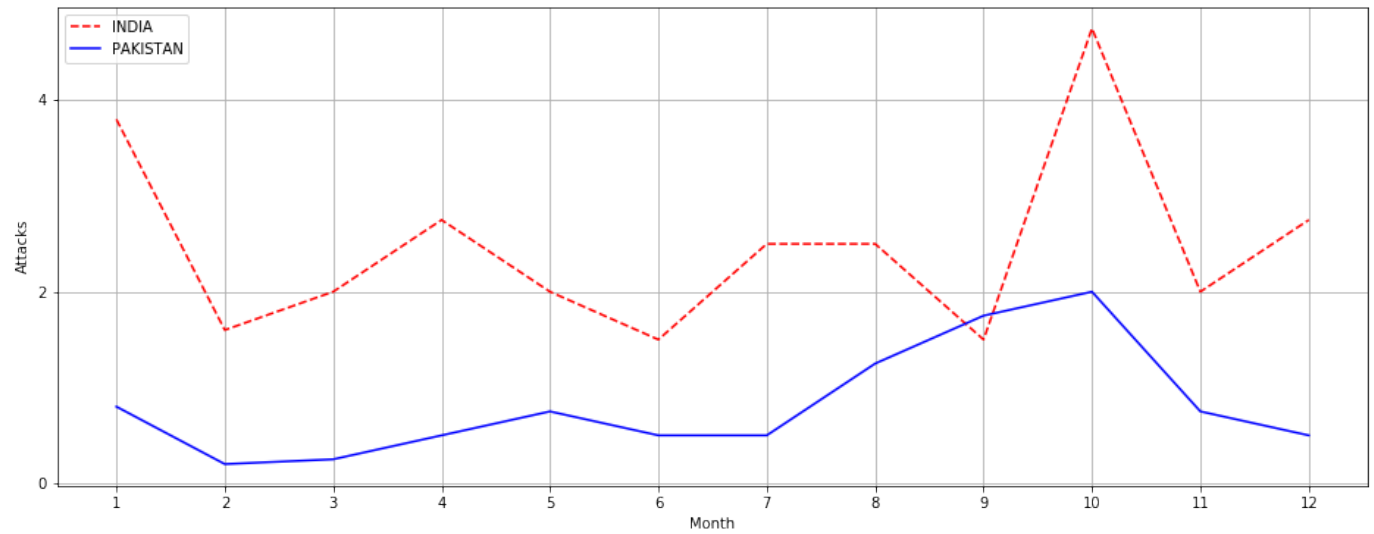

Figure 5.9: Average Cyber Attacks / Month for India and Pakistan.

bursts in cyber attacks drastically affect the average. Though the average peaks in October, October was the peak in only 2 years, as shown in 5.14. In 2014, it peaks in October with 6 attack recorded, more than 3 times the per month average of 1.74 attacks. Additionally, in 2016, the peak is 1 attack recorded, where October shares the maximum with

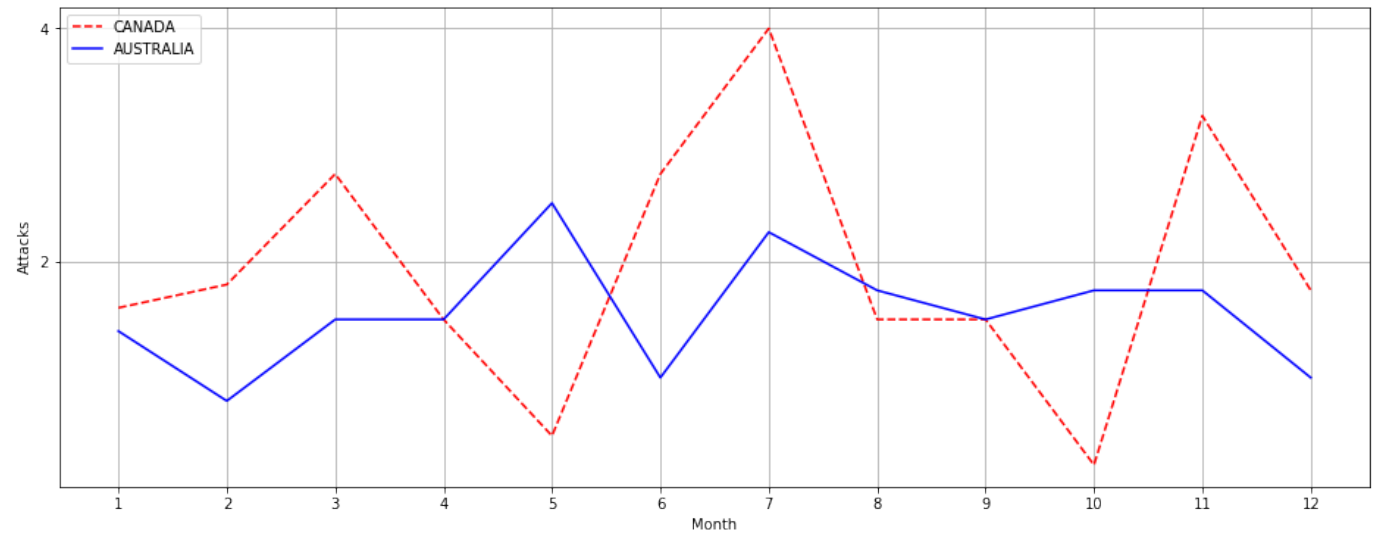

Figure 5.10: Average Cyber Attacks / Month for Canada and Australia.

\subsubsection{Correlates}

Table 5.1 shows the correlation between attacks received volume between the top twenty attack target countries. The correlation table indicates which countries are 
likely to have similar changes in attack volume during the same month. The reason may be retaliatory, i.e. country 'A' and country ' $\mathrm{B}$ ' may see attacks received increase at the same time because they are attacking each other. The correlation may also have a common cause, in the form of political, social, economic instability, or a common aggressor. This data is of course, not all the data that can be produced with this new cyber attack database, but produces 190 useful Pearson R correlations. A $20 \times 20$ correlation matrix render 400 entries, but 20 values along the diagonal are all $r=1.00, p=0.00$, because any item is $100 \%$ correlated with itself with absolute significance, and the entries on one side of the diagonal are duplicated on the other side of the diagonal.The $r$ value is the Pearson correlation, where the absolute value determines how closely they are correlated. This is previous discussed in 3.7. Positive $r$ values indicate that in general an increase in attacks in one country translates to an increase in attacks on another country, and negative $r$ values indicate that an increase in attacks on one country translates to a decrease in attacks against another country, and vice versa. As discussed in 3.7, p-values are always positive, and the smaller the more significant, where a value less than 0.05 is statistically significant. The table is sorted in ascending order by p-value, which indicates statistical significance. However, this is not the only thing we care about. For instance, if two countries both have zero cyber attacks reported for the entire 4 years, then they would have a perfect correlation, with absolute significance. The most correlated countries, with the greatest significance, are Turkey and the the Philippines in the top row of 5.1, with a total attack volume over the 4-year period of 36 and 22 attacks, respectively. Here we may want to focus on countries that have a large to moderate volume of cyber attacks, and see how they correlate. The US and China are both in the top 5 target countries, and we can see that they are moderately correlated in attack volume $(r=0.309, p=0.029)$.

In the figure 5.11, Turkey and the Philippines have very few attacks, but their 
attack volume is correlated. Out of all countries, it is in fact the most correlated $(r=0.467, p=0.001)$. Though Turkey has a global maximum of 4 attacks in January 2014, its next highest attack month is November 2014. Conversely,the Philippines has a global maximum in November 2014, and its second highest attack month is one month after Turkey's. So, though their $1^{\text {st }}$ and $2^{\text {nd }}$ maxima do not perfectly align, the collectively occupy a similar temporal space, and behave similarly over the 4 year period. It is therefore highly unlikely that their correlation is merely due to their both receiving a low attack volume. Explaining the reasons for many of these correlations is difficult, but shall be discussed in the Discussion section 6 .

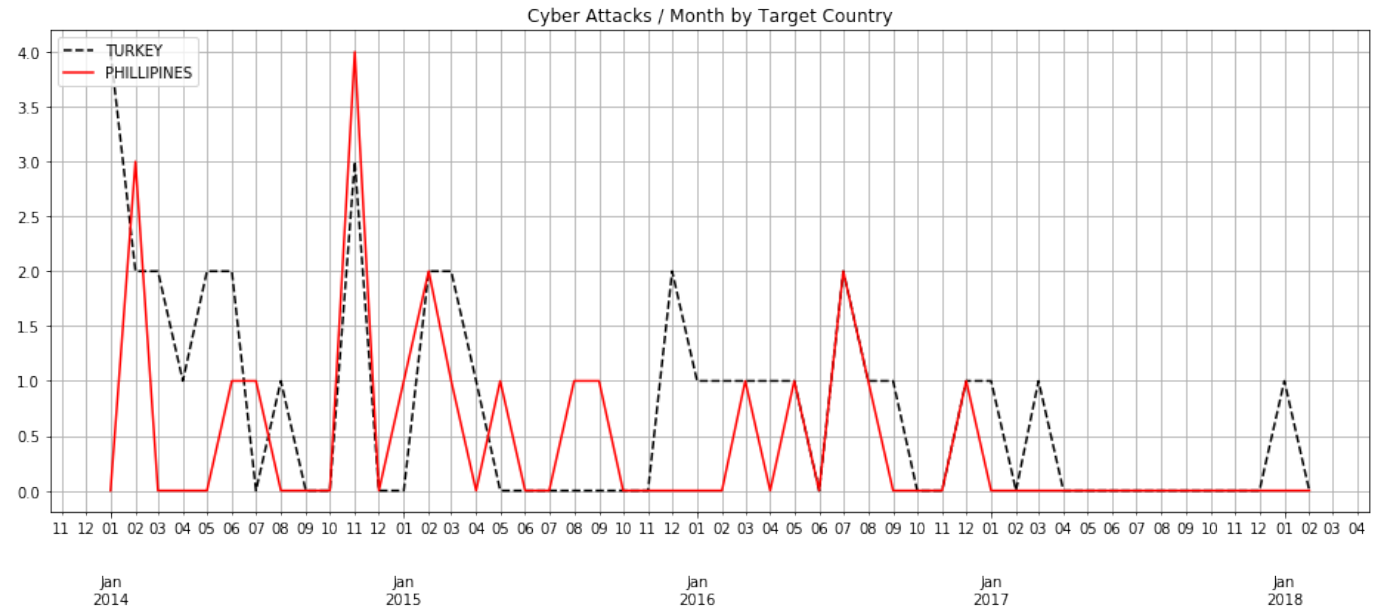

Figure 5.11: Timeline of cyber attack volume by country for Turkey and the Phillipines.

Another one of the strange results of this research is in figure 5.12. It is surprising to see that Canada and Pakistan are weakly inversely correlated $(r=-0.294, p=$ 0.038) as shown in table 5.1. For much of the graph in figure 5.12 Canada's attacks will increase while Pakistan's will decrease, and vice versa. Possible reasons for this, and other correlations will be discussed in the Discussion section 6 .

I show that Japan and China's reported attack volume in figure 5.13. As shown in table 5.1, China and Japan are moderately correlated $(r=0.339, p=0.016)$. In the graph we can qualitatively notice that the volume of attacks received by Japan 


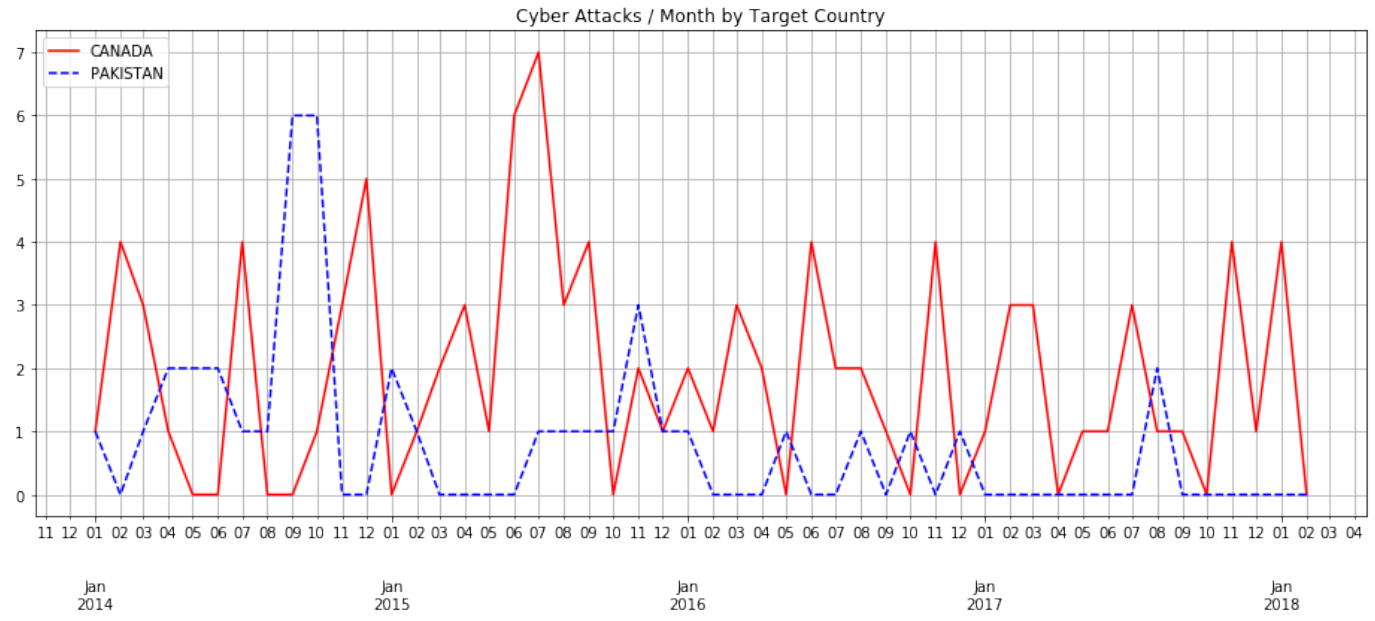

Figure 5.12: Timeline of cyber attack volume by country for Canada and Pakistan.

generally increases and decreases with those of China. The unique maximum of attacks received by China (5 attacks) occurs at the same month, January 2016, as one of Japan's 3 maxima (3 attacks) over the entire 4 year period.

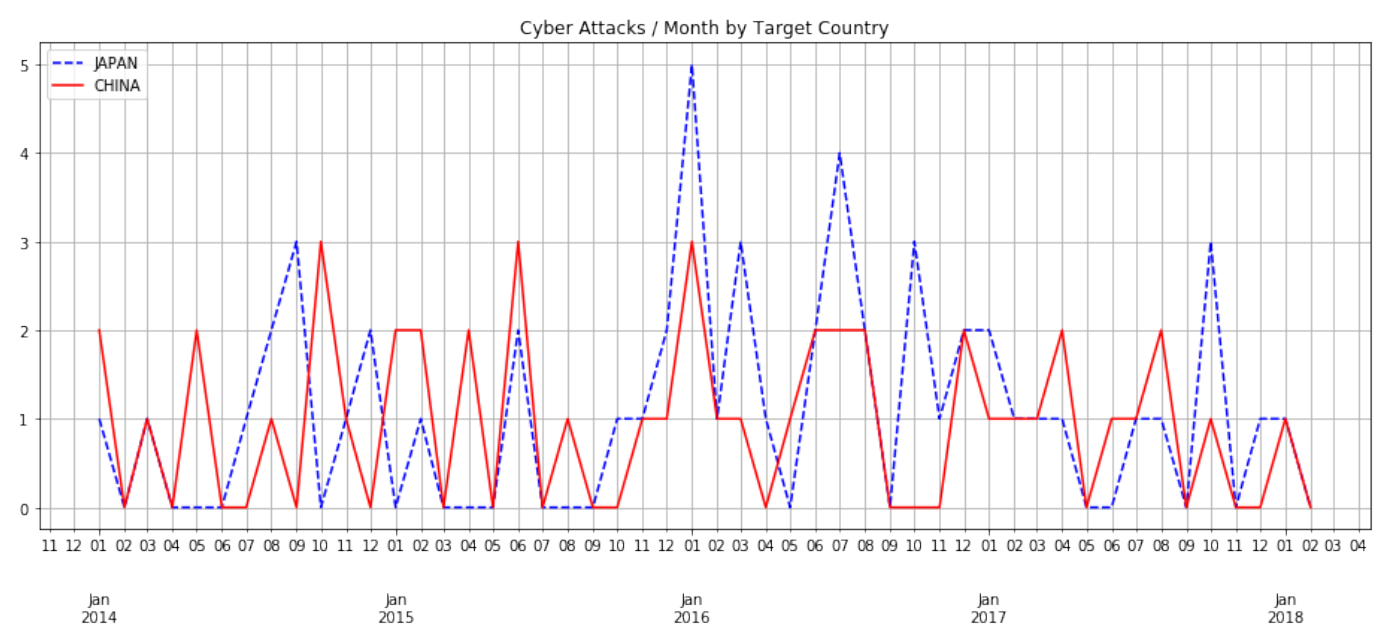

Figure 5.13: Timeline of cyber attack volume by country for Japan and China.

As with the previous graph, in figure 5.14, we see that Pakistan and India both share one of their 2 global maxima in the same month, October 2014. Similarly, Pakistan's 2015 maximum occurs 1 month after India's, and in 2017, Pakistan goes 
from 7 months of zero reported attacks to receiving 2 attacks in August, the same month that India has its second greatest attack attack volume. 2016 is somewhat less interesting, because Pakistan only has 5 months of one attack and 7 months of zero attacks. However, in 2016 both India and Pakistan share a maximum in December. Running the Pearson correlation, the attacks received by Pakistan and by India are at least weakly correlated, $(r=0.289, p=0.042)$, as one might expect from the previous visual inspection.

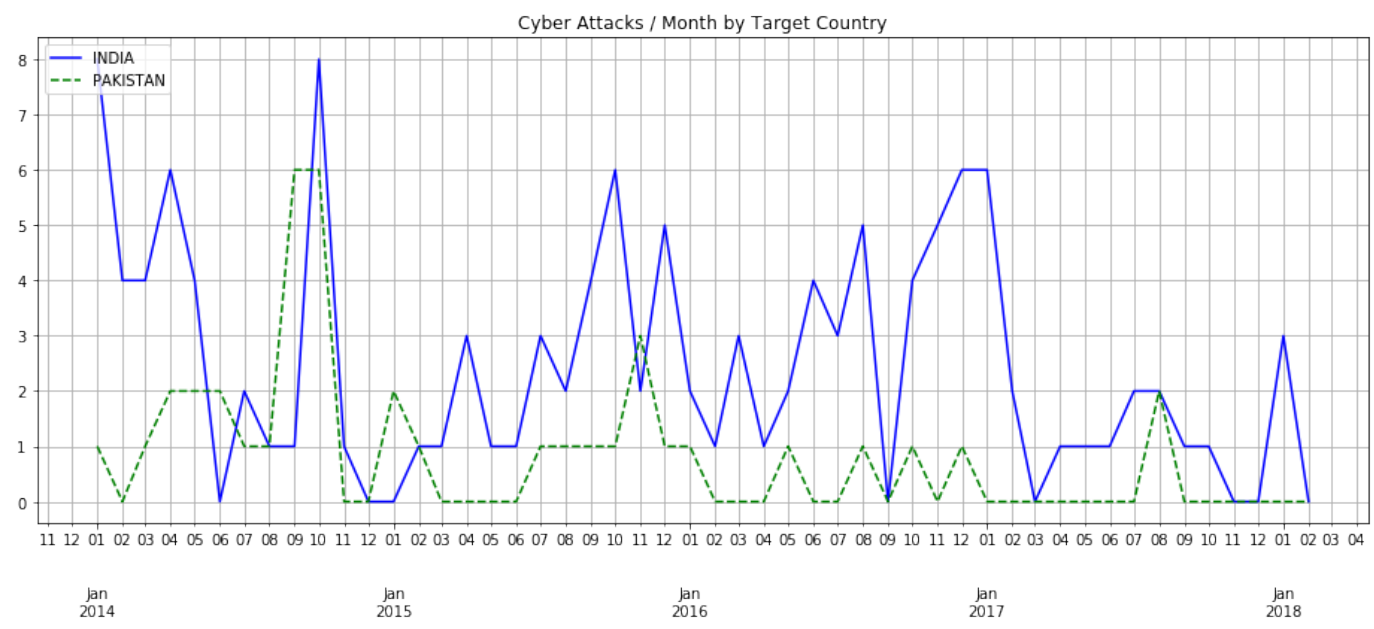

Figure 5.14: Timeline of cyber attack volume by country for India and Pakistan.

I show Italy and Germany in figure 5.15 are also moderately inversely correlated $(r=-0.356, p=0.011)$. Graphically we can notice that Italy reaches its global maxima of 3 attacks per month Nov 2014 and Feb 2016, while Germany has zero recorded attacks. Similarly, as German reaches its maximum of 3 attacks per month in August 2016, Italy has zero recorded attacks. Similar behavior is easily seen for several nthmost voluminous months, thus giving some intuitive understanding to their moderate inverse correlation. In general, there are surprising and often inexplicable correlations

The US and China can figure 5.16. Their correlation is more difficult to visualize because of the order of magnitude difference in attacks reported. They neither share any global maxima, nor do they share any maxima for the year. However, the US 


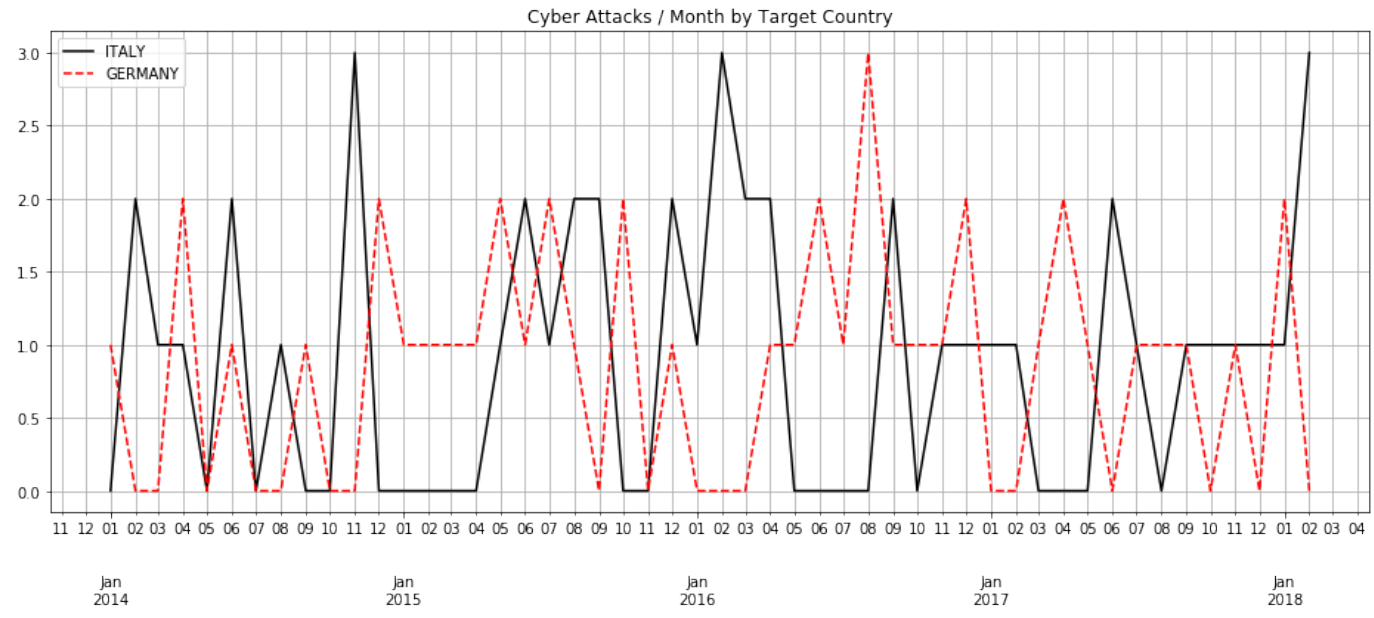

Figure 5.15: Timeline of cyber attack volume by country for Italy and Germany.

and China do share global minima in May 2015 and Nov 2017 (US = 21, CN =0). They are moderately correlated $(r=0.309, p=0.024)$

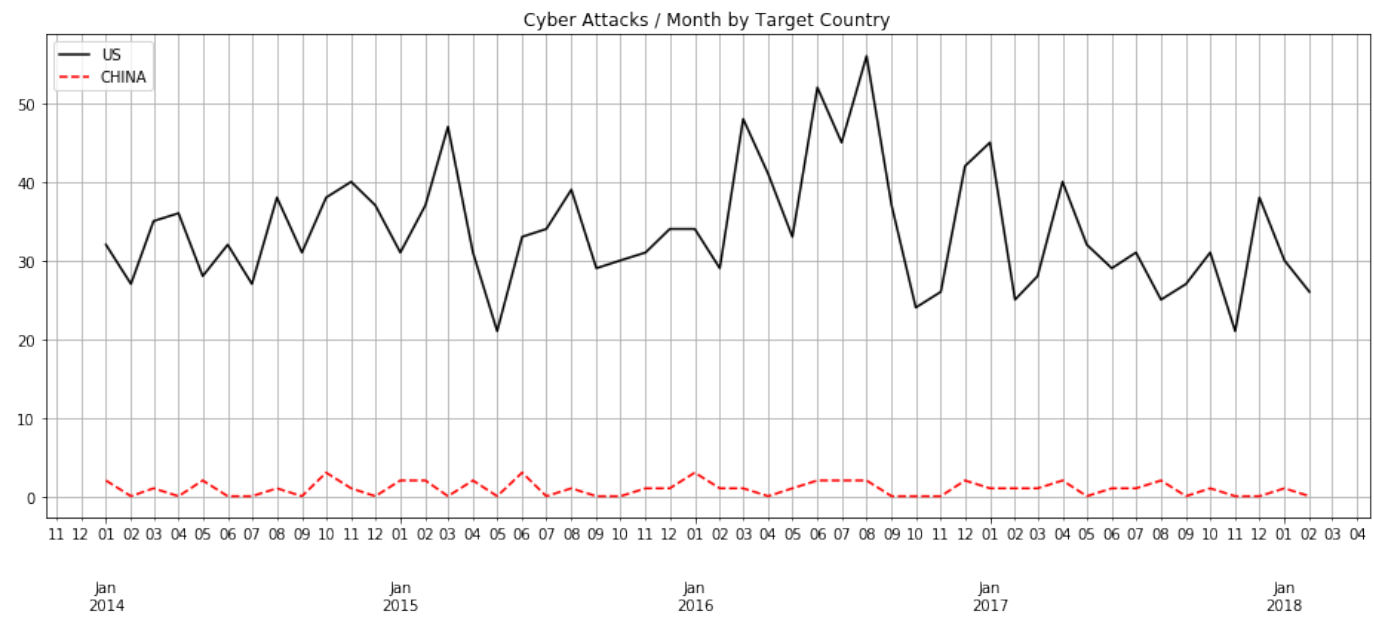

Figure 5.16: Timeline of cyber attack volume by country for the US and China.

Japan and China are also moderately correlated $(r=0.339, p=0.016)$. This can be visualized in figure 5.13. Japan and China both share a maximum of received attacks in January 2016, as well as a second-highest attack month in July 2016, and a third-highest attack month in October 2017. However, they are sometimes very out 
of synce, as in Sept 2014 and October 2016, where Japan reports 3 attacks and China reports none, or in Oct 2014 in which China reports 3 attacks and Japan reports none.

\subsection{By Sector}

Sectors are defined by the "TargetClass" field of the database, originally from Passeri's spreadsheets column name "Target Class" as previously discussed 3.2. There are a total of 629 distinct values of TargetClass. They often have prefixes of "Industry" or "Org", which can help organize them better, such as "Industry: Video Games", or "Org: Telecommunication". It often does not seem that Industries and Organizations are clearly delineated. It seems possible, for instance, to call "Industry: Telecommunication", "Org: Telecommunication", and vice versa. There are 109 distinct Org entries, and they account for 309 attacks. There are 306 distinct Target Classes with the "Org" prefix, and 1,078 attacks with an Attack Class containing an "Industry" prefix. Together, that is 1,384 attacks, $36 \%$ of the entire database. To this researcher, there seems to be small difference qualitatively between the categorizations of "Industry"-prefixed and "Org" -prefixed target classes: the former is associated with private business, whereas the latter generally refers to political, religious, or nonprofit groups. In monthly attack volume, they are moderately correlated $(r=0.476, p=0.000)$, as shown in 5.3. Graphically, they are also fairly similar, as shown in figure 5.17. Besides the "Org" and "Industry" prefix ambiguity, there is ambiguity and overlap among sectors themselves. For instance, the TargetClass labels "News", "News (Bitcoin)", "Industry: News" and "Industry: News and Publishing" are all different labels that ostensibly reference an attack against the same sector. Additionally, some attacks target multiple sectors, in which the "TargetClass" is recorded simply as "> 1", omitting any information about the particular sectors 


\begin{tabular}{|c|c|c|c|}
\hline Country A & Country B & $r$ & p-value \\
\hline TR & $\mathrm{PH}$ & 0.458 & 0.001 \\
\hline $\mathrm{AU}$ & IT & -0.366 & 0.009 \\
\hline IT & $\mathrm{DE}$ & -0.356 & 0.011 \\
\hline JP & $\mathrm{CN}$ & 0.339 & 0.016 \\
\hline US & JP & 0.326 & 0.021 \\
\hline SA & $\mathrm{CN}$ & 0.324 & 0.022 \\
\hline IT & PK & -0.318 & 0.024 \\
\hline US & $\mathrm{CN}$ & 0.309 & 0.029 \\
\hline $\mathrm{RU}$ & JP & 0.305 & 0.031 \\
\hline IL & PK & 0.297 & 0.037 \\
\hline $\mathrm{CA}$ & PK & -0.294 & 0.038 \\
\hline IN & PK & 0.289 & 0.042 \\
\hline IN & $\mathrm{CN}$ & 0.268 & 0.059 \\
\hline $\mathrm{KR}$ & NL & 0.268 & 0.059 \\
\hline US & $\mathrm{TR}$ & 0.265 & 0.063 \\
\hline IN & TR & 0.264 & 0.064 \\
\hline IL & IT & -0.264 & 0.064 \\
\hline US & $\mathrm{DE}$ & 0.251 & 0.079 \\
\hline US & $\mathrm{RU}$ & 0.246 & 0.086 \\
\hline FR & PH & 0.244 & 0.088 \\
\hline
\end{tabular}

Table 5.1: The top 20 country-to-country correlation and p-values of the top 20 targets of cyber attacks, arranged in ascending order by p-value. The full list of cross country correlations is in table 5.1 


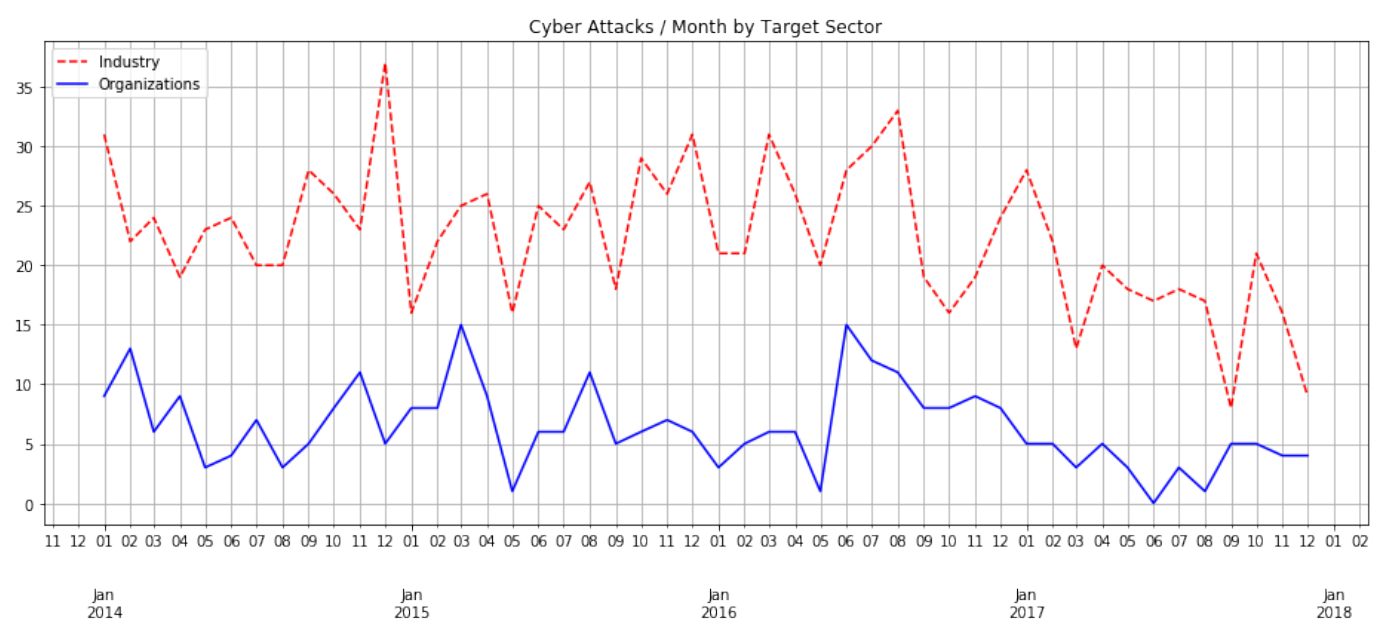

Figure 5.17: Attacks Timeline for Industry and Org Sectors.

affected, which seems unhelpful. These inconsistencies makes data analysis against target sectors very difficult. For this reason, I focus mainly on the sectors in table

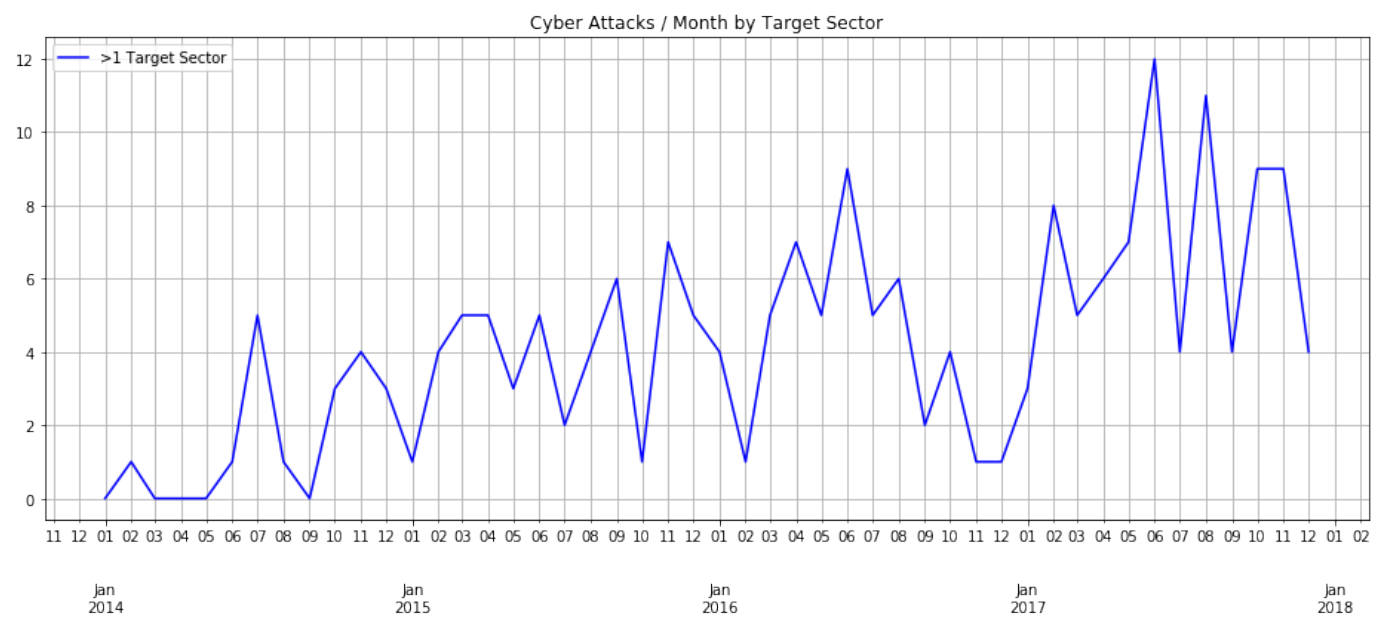

Figure 5.18: Attacks Timeline for $>1$ Label

5.2 , excluding the label "> $1 "$.

I now explain why "> 1 " is not very helpful in this particular analysis. The label " $>1$ " is the $6^{\text {th }}$ most targeted sector label, but it is not correlated in any statistically significant way to any of the most targeted sectors, nor is it correlated with total attacks. This indicates that attacks of a broad scope are not randomly 
distributed amongst all attacks, but they are also independent of attacks against any other significantly targeted sector. They represent a significant portion of cyber attacks, but do not occur in similar frequencies to other popular target sectors; broad attacks are their own beast entirely. For reference I show their behavior in figure 5.18. They could be analyzed by other means, such as the type of attack, "Attack", such as "DDOS", "Defacement", or by "AttackClass", such as "CC" (Cyber Crime), or "CW" (Cyber Warfare). But broad attacks do not seem to be related to any other important target sectors. This cyber attacks database supports a near infinite combinations of queries for knowledge discovery, but only a fraction of them are presented in this thesis.

\begin{tabular}{|c|c|}
\hline Sector & Total Attacks \\
\hline Various Industries & 1078 \\
\hline Government & 592 \\
\hline Individuals & 440 \\
\hline Org & 306 \\
\hline Education & 245 \\
\hline$>1$ & 198 \\
\hline Health Care & 175 \\
\hline Finance & 167 \\
\hline News & 94 \\
\hline
\end{tabular}

Table 5.2: The 9 most targeted sectors between January 2014 and February 2018, arranged in descending order by attack volume.

Perhaps these ambiguities will be ameliorated in future reports, as Passeri has decided to make future reports in line with the International Standard Industrial Classification (ISIC), as stated in his Feb 2018 blog post [61]. Indeed, this new classification scheme seems to be the case in all of the cyber attacks Passeri has 
recorded for 2018 so far, as shown by querying the attack database I have created with Passeri's data, as shown in figure 5.19. The effort undermines itself because it includes both the ISIC code and its associated label. If this is changed, it will greatly aid future studies, but for now, the vast majority of data collected uses the older ad hoc naming convention. Because the sector labels have changed starting January 2018, I present data only from January 2014 through December 2018.

\begin{tabular}{|c|c|c|c|c|c|c|}
\hline Id & AttackDate & Author & Target & Description & Attack & TargetClass \\
\hline 41664 & $2018-01-01$ & BULL & Fave Brookes & 2018 beains with a new roun... & Unknown & X Individual \\
\hline 41665 & 2018-01-01 & 패느 & Rockinaham Countv ... & Rockinaham Countv Schools ... & Malware & P Education \\
\hline 41666 & $2018-01-02$ & Andariel & Unnamed South Kor... & Bloombera reveals that a ha... & Unknown & Z Unknown \\
\hline 41667 & 2018-01-02 & $(a 0 \times 55 \ldots$ & theflv.com & A hacker usina the twitter h... & SOLi? & J Information and communication \\
\hline 41668 & $2018-01-03$ & FULL & Uber Users & Svmantec researchers disco... & Malware & $\mathrm{X}$ Individual \\
\hline 41669 & $2018-01-03$ & सOLL & Android Users & Researchers from Trend Micr... & Malware & $\mathrm{X}$ Individual \\
\hline 41670 & 2018-01-03 & स्सLL & Citv of Farminaton & The citv of Farminaton is hit ... & Malware & O Human health and social wor... \\
\hline 41671 & 2018-01-03 & HULL & Linux Servers & Researchers at F5 discover ... & Malware & $\mathrm{X}$ Individual \\
\hline 41672 & $2018-01-03$ & स्Lा & Bank customers alob... & Researchers from securitv $c . .$. & Malware & $\mathrm{X}$ Individual \\
\hline 41673 & $2018-01-03$ & सULL & Bia Line Holidav & Bia Line Holidav, a Hona Kon... & Malware & R Arts entertainment and recre... \\
\hline 41674 & 2018-01-04 & सOLL & Ukrainian users & Researchers from Cisco Talo... & Malware & $\mathrm{X}$ Individual \\
\hline
\end{tabular}

Figure 5.19: ISIC Compliant Target Classes

Though the attacks have now been put into a database, the ad hoc naming convention previously used for the "TargetClass" column makes querying the data difficult. Many sectors have been well-delineated, and those that have not have been grouped into the "Industries" and "Org" categories as previously described. Though "Industry" and "Org" are only prefixes to the specific sector, such as "Telecom", erring on the side of inclusion helps to make inferences on a grander scale, such as the threat to the private sector in general. To get around this inconsistency, targeted queries similar to figure 5 are used to get accurate counts of attacks against a particular industry.

To understand how attack volume against one sector is related to others, I have created a correlation matrix containing $r$ and $p$ values, as done in the previous section. As I have previously described the creation of the countries correlation table, table 5.1, I remove the diagonal, and the duplicated data on one side of the diagonal. After arranging the correlations in a columnar layout in descending order of $p$ value, 
table 5.3 is the result. Because of the complex nature of the motivation behind cyber attacks, one cannot say that the volume of cyber attacks that one sector receives is the cause or effect of the volume of cyber attacks that another sector receives. However, this study allows us to see the shifting priorities of black hat hackers in general.

One insight we can gain from table 5.3 is that the attacks received by individuals and the attacks received against various industries are strongly inversely correlated $(r=-0.595, p=0.000)$, and are actually the most strongly correlated sectors, by absolute value. As the private sector in general experiences fewer cyber attadcks, individuals experience more, and vice versa. This can be visualized in figure 5.20. As

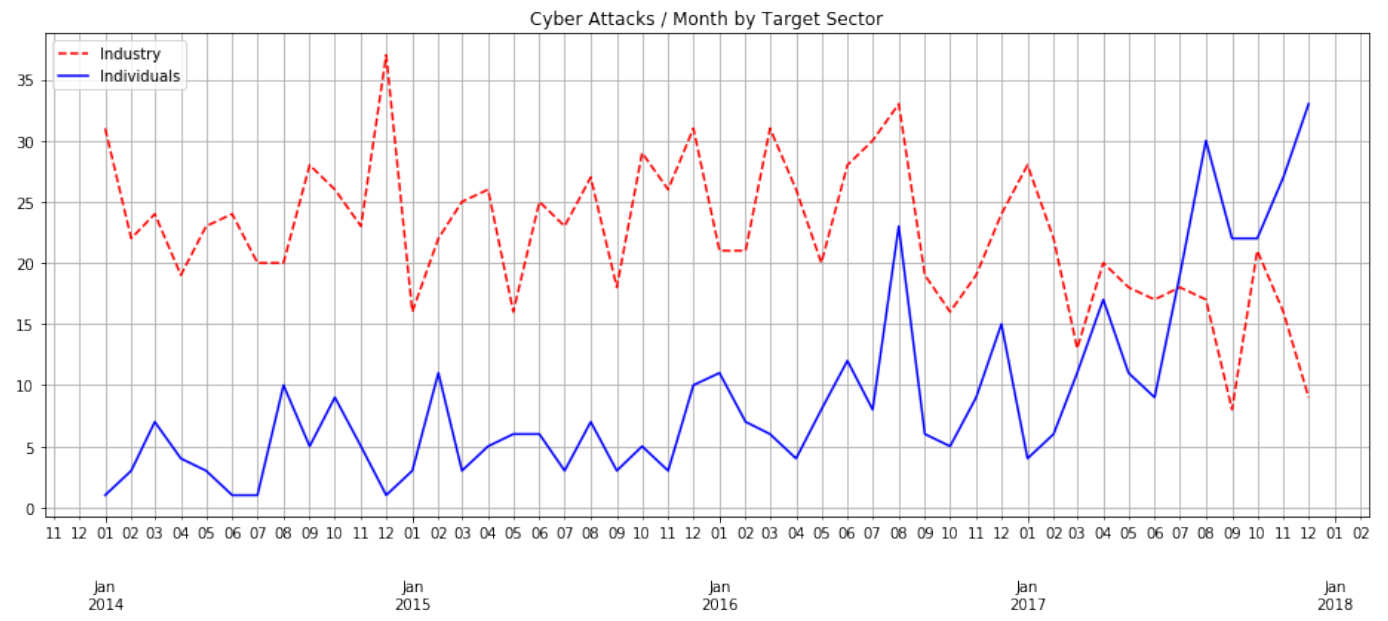

Figure 5.20: Attacks Timeline for Attacks Against Individuals and Industry Sectors

I show in this figure, there is an overall trend of attacks against industry decreasing while attacks against individuals increase. In addition to this long view of the behavior, there are also months in which the cyber attacks against industry and cyber attacks against individuals are at odds. For instance, in December 2014 industry sees a global maximum of attack volume, and individuals are near a global minimum. In May 2015, attack volume against industry reaches a local minimum as attack volume against individuals reaches a local maximum. The converse is true in June 2014. In 
August 2018, the month in which overall cyber attacks peaked over the 4 year period, both attacks against industry and attacks against individuals reach a local maximum. Afterwards, they start diverging, and by July 2017, attacks against individuals have overtaken those attacks against industry.

The $2^{\text {nd }}$ strongest correlation, shown in table 5.3 is between individuals and the health care sector. This is also the strongest positive correlation. In figure $5.21 \mathrm{I}$ compare their monthly attack volume. They both share a global maximum in December 2017, local maxima in August 2014, January 2016, August 2016 (at the peak of all cyber attacks), and April 2017. Though they are strongly correlated and have both grown on average, attacks against individuals have grown much more quickly; starting March 2017 through the end of the year, individuals have received at least twice as many cyber attacks as the entire health care industry. This may be because of the similar goals between attacks against health care institutions and individuals, namely personally identifiable information (PII). Out of the 175 cyber attacks against the health care sector, 42 were of the of the attack class ("Attack" column) "Account Hijacking", or $24 \%$. This ratio with respect to attacks against individuals is $35 \%$, whereas in industry, a target sector inversely correlated to individuals, only $10 \%$ of all cyber attacks are via account hijacking. Evidently, the type of attack varies greatly between sectors, and health car and individual targets are similarly attacked for user account information.

The Industry and Government sectors are also strongly correlated $(r=0.548$, $p=0.000)$ in table 5.3. I show in figure 5.22 the monthly attack volume against both sectors. Both sectors seem to be y-shifted versions of one another from January 2014 - July 2014, and then start to exhibit more independence. Overall, both rapidly declined since January 2017, in contrast to the previously discussed attacks against individuals, which have skyrocketed. However, total cyber attacks overall have not behaved this way, as previously shown in figure 5.1. What naturally emerges from 


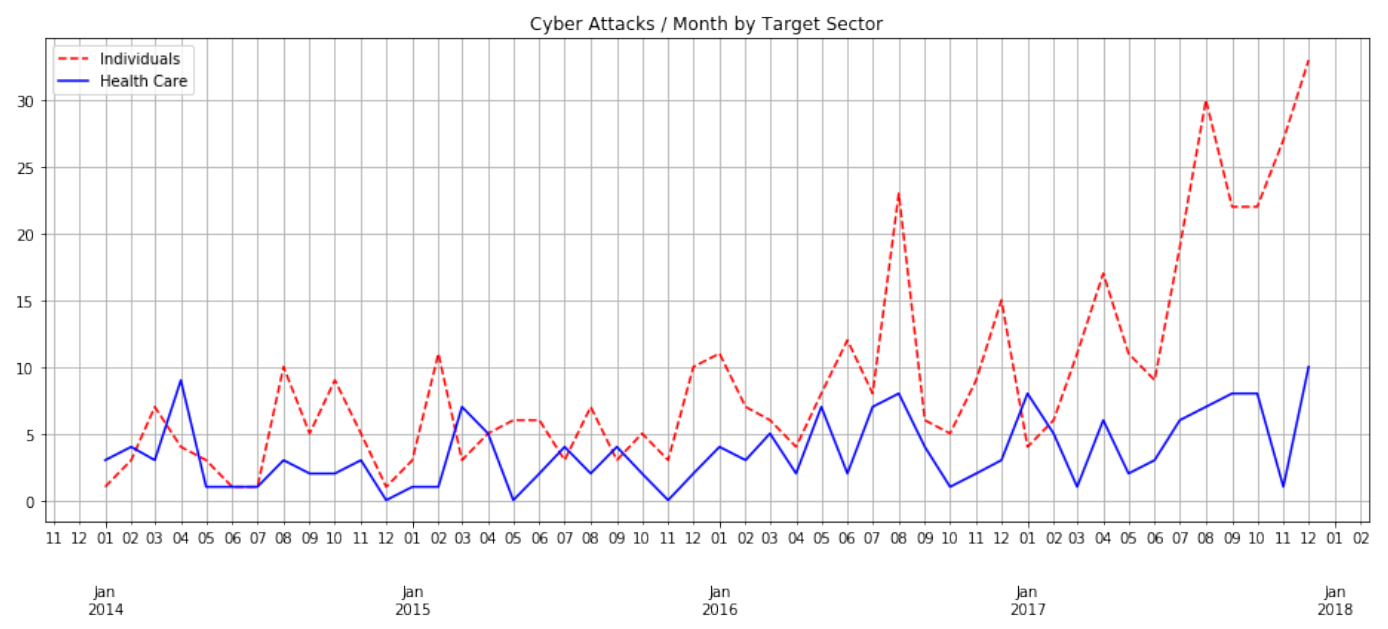

Figure 5.21: Attacks Timeline for Individuals and Health Care sectors

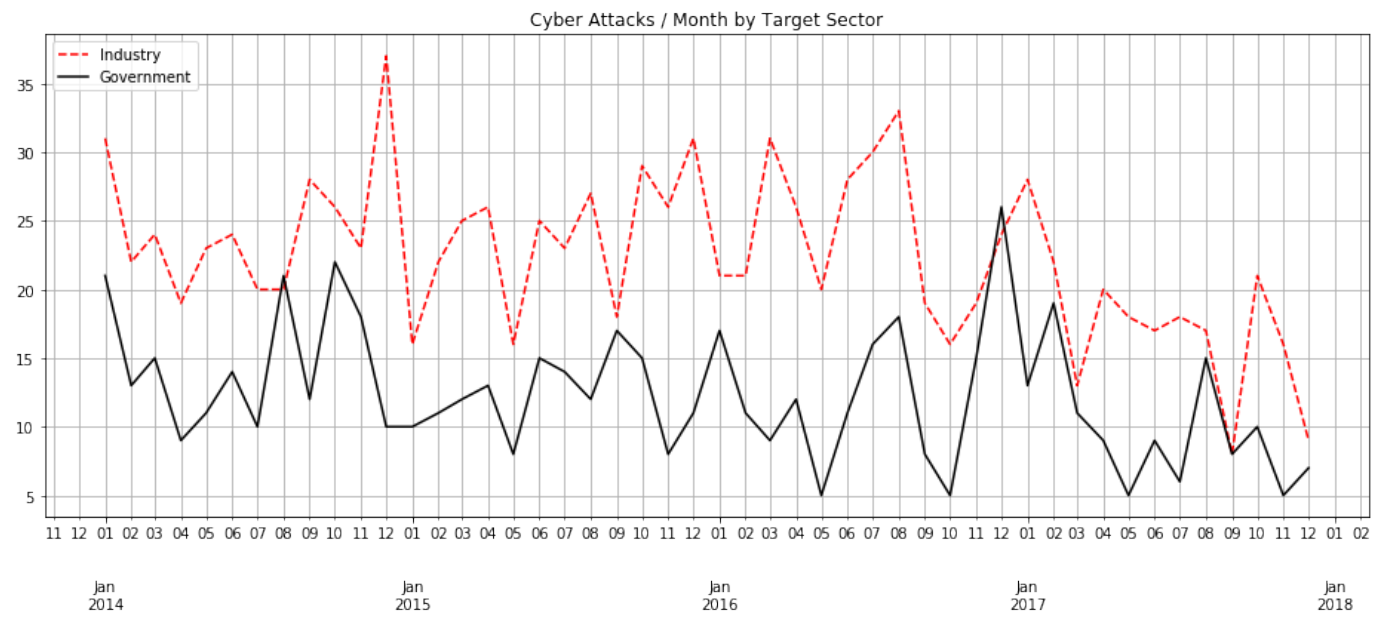

Figure 5.22: Attacks Timeline for Government and Industry Sectors, the top 2 targeted sectors.

these data is the notion of a loose conservation of cyber attacks; as one victim is ignored to some degree, others are targeted more frequently and to similar degree. Of course, this is a loose conservation, because the total volume of cyber attacks also fluctuates, but the targets, be they sectors or countries, fluctuate much more, often in complementary fashion, when there exists at least a moderate inverse correlation of attack volume between targets, as government and industry are in figure 5.23. . In general, it seems that hackers are targeting industries and governments less and 


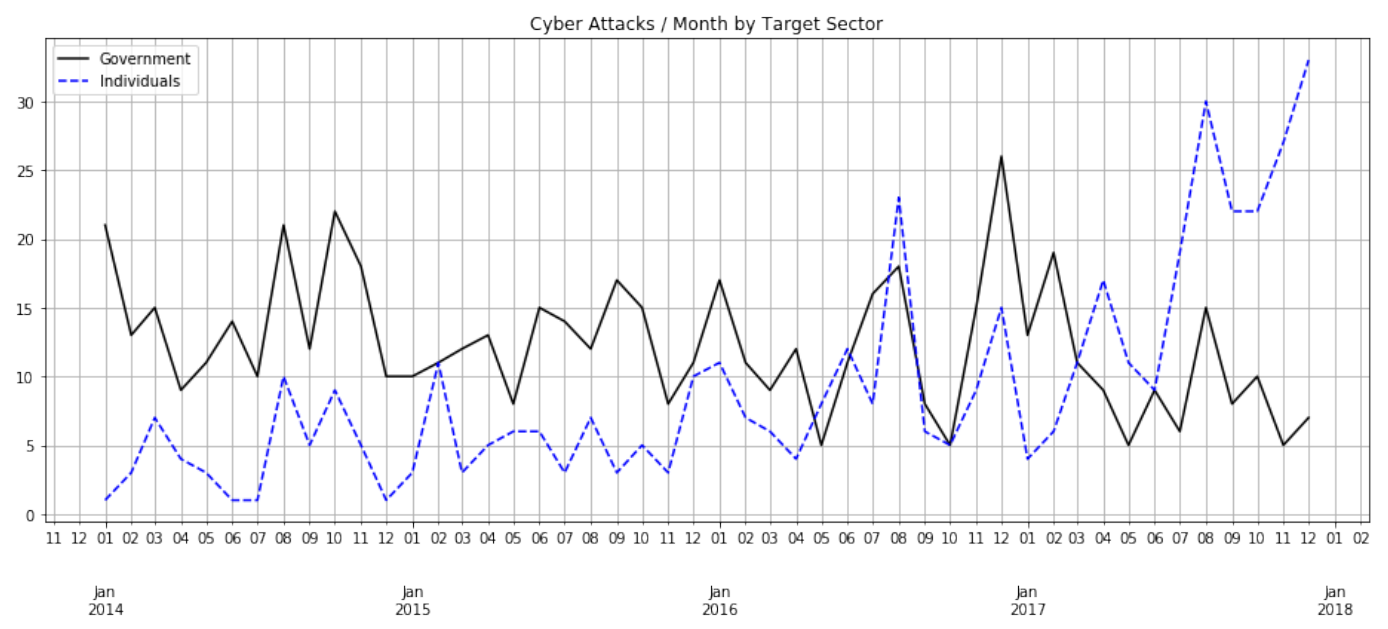

Figure 5.23: Attacks Timeline for Government and Individuals.

targeting individuals more.

Attacks against individuals and the financial sector are also moderately correlated $(r=0.446, p=0.001)$. Their monthly totals are shown in figure 5.24. . Their

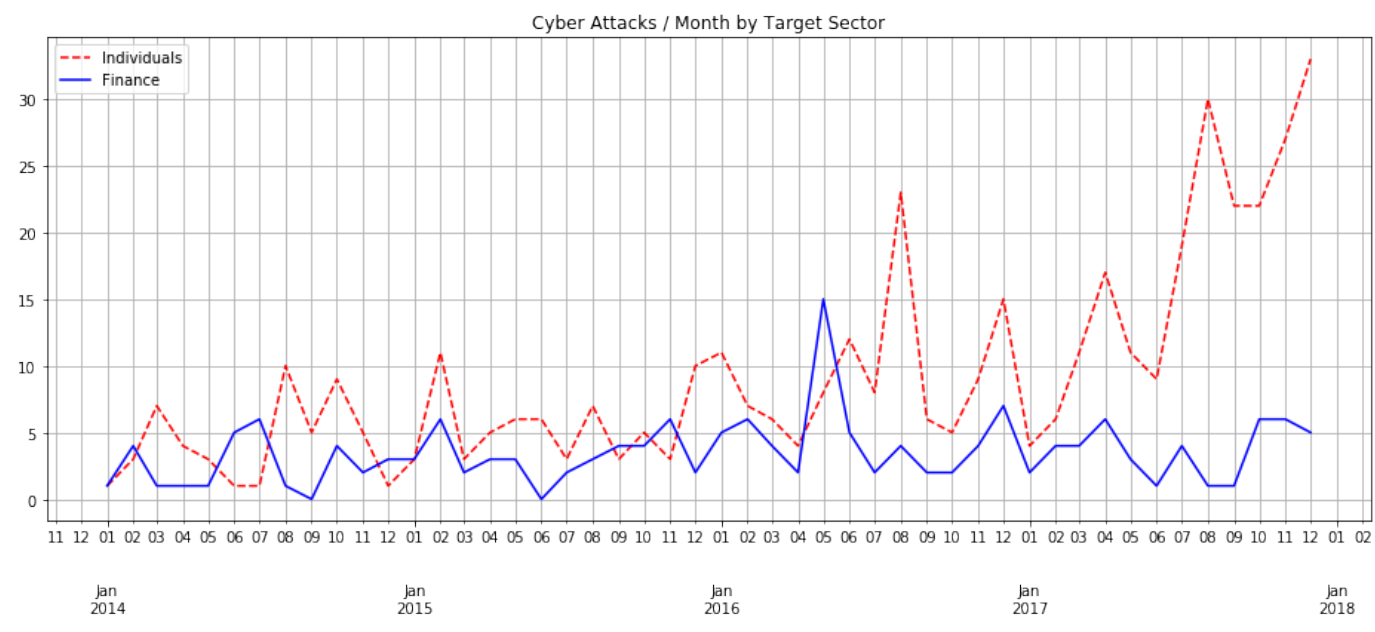

Figure 5.24: Attacks Timeline for Individuals and Finance sectors

correlation is somewhat more difficult to see, as the locations of their local minima and local maxima often do not align. All that can be said is that when individuals are attacked more frequently, the financial market is often attacked more frequently as well. May 2016 represents the highest attack against financial markets over the 4 year 
period, in which it experienced 15 attacks, more than 4 times the average monthly attack rate. At least 3 of these attacks were against the SWIFT banking network, whose total losses from attacks 2015-2016 are in the millions [27]. SWIFT came under greater scrutiny after $\$ 81$ million was stolen from one of its banks in Bangladesh in February 2016 [27].

\subsection{Socioeconomic Factors}

The results of analyzing the correlation between socioeconomic factors of a country and the total cyber attacks received imply that no one factor is even moderately correlated to cyber attack volume. The origin and meaning of these socioeconomic factors is previously discussed in section 4.1. This corroborates the findings of Solano's "Socio-economic factors in Cybercrime" [64]. Solano's work examines more specific relationships between socioeconomic factors of individual countries and their cyber attack volume. I find that A few socioeconomic measures are weakly correlated with cyber attack volume, as I show in table 5.4. Population $(r=0.208, p=0.009)$ and GDP $(r=0.179, p=0.027)$ are the factors most correlated to cyber attack volume. As GDP and population both represent resources for an entire country, this makes sense. In contrast, other socioeconomic factors which are not correlated to cyber attack volume, such as generosity $(r=0.133, p=0.125)$, or the belief that people can be trusted $(r=0.134, p=0.335)$, represent qualities of a group, but are not considered resources.

Because the US is so anomalous in its extreme volume of cyber attacks, it can be helpful to see how socioeconomic factors correlate with cyber attack volume with the US removed. The result is figure 5.5. Comparing this table to the previous one, the US changes things considerably. By simply removing the US, the correlation between population and cyber attacks received jumps from 0.208 to 0.360 , and its 
p-value is decimated. Removing the US also takes the correlation between healthy life expectancy from $r=0.131, p=0.114$ to $r=0.299, p=0.000$, and similar effects are found in other correlates as well. This suggests that certain socioeconomic conditions of the US make are not as correlated to cyber attack volume as the same conditions are in other countries. Removing the US as an outlier can help see trends it obscures.

The lower and upper confidence intervals of the happiness score shed some light on how the distribution of happiness scores correlate to cyber attack volume received. The narrower the interval is, the more uniform the distribution is. The lower confidence interval's positive correlation with cyber attacks received could be interpreted as a side effect of either a higher happiness score or a narrower distribution of happiness scores. To examine this idea more closely, I analyze the correlation between the size of the confidence interval of happiness score (Higher Confidence Interval of Happiness - Lower Confidence Interval of Happiness) and cyber attacks received. The result is $r=-0.242, p=0.003$. This suggests that there is at least a weak inverse correlation between the size of the confidence interval for happiness and cyber attacks. If the happiness interval is very small, it means that most surveyed report similar happiness levels. Thus, societies in which most people are similarly happy have smaller confidence intervals of happiness, and receive more cyber attacks. This may be a counterintuitive example, as one may expect large confidence intervals with regard to happiness to correlate with income inequality and other social inequalities, which may in turn correlate to universally deleterious outcomes, of which cyber attacks are one. The idea that greater variance in happiness correlates with fewer cyber attacks may be explained by more complex socioeconomic forces. For instance, a wider confidence interval of happiness moderately correlates with the sentiment that people can be trusted with values $r=-0.445, p=0.002$. And the sentiment that people can be trusted is moderately correlated with cyber attack volume. Intuitively, it can be observed that the more disparity between happiness recorded, the less people trust 
each other. In a dystopia it could be the case that a few powerful people are very happy, and the rest are very unhappy, in which case the confidence interval would be very large, and people would not trust each other very much. In general, when these results seem unintuitive, one possible way to understand these correlations may be indirect results which can be explained by other more intuitive correlations. For another example, the happiness confidence interval size is inversely correlated with healthy life expectancy at birth $(r=-0.221, p=0.008)$, which in turn is correlated with increased cyber attacks $(r=0.299, p=0.000)$. This is more intuitive, because a greater life expectancy is generally associated with wealthy countries who experience more cyber attacks. This intuition is confirmed in that life expectancy is strongly positively correlated with the $\log$ GDP per capita with $r=0.811, p=0.000$. Many of these seemingly disparate socioeconomic features of a society indicate the assets that a society has, and therefore, the value of launching a cyber attack.

Another important aspect about socioeconomic conditions is the unique patterns to attacks against certain countries. For instance, in 3 of 4 years in question in this study, Great Britain received its peak cyber attacks in November, as previously shown in figure 5.4 implying a heightened risk to GB leading up to holiday shopping season. In 2016 Great Britain had 2 maxima: one in the predictable November spot, and one in June, when the Brexit vote was held [39], it is possible that it is coincidental, or that general social tension is correlated with a greater volume of cyber attacks received.

Election cycles can also play a large role in cyber attack volume. India's most recent general election occurred in April - May 2014 [62], and 3 months prior to this, India's cyber attack volume reached a maximum for the entire 4 year period, also illustrated in figure 5.4. The US also experienced a similar influx of cyber attacks at a similar time relative to a major election. Over the 4 year period, cyber attack volume against the US reached a maximum 3 months before the November 2016 
election, as shown in figure 5.2.

Alternatively, some attacks do not aim to change an election outcome, but to protest it. As shown in figure 5.6, Israel's maximum cyber attacks received occurs in April 2015. In 2 ways these attacks against Israel are different than both the attacks around the Indian and American elections. Firstly, the individual attacks are clearly politically motivated by their descriptions. Of the 9 attacks in April 2015, 6 attacks are coordinated under the \#OpIsrael tag, 1 is carried out by the Palestinian hacker group "Gaza Team" defacing government sites with pro-ISIS propaganda, 1 attacks Israeli military networks, and 1 attacks the Israeli arms importer and manufacturer Fab-Defense. Secondly, these attacks follow the election, rather than precede it. These attackers are not trying to influence an election or taking advantage of the socioeconomic condition for profit; they are likely protesting the recent controversial election of Prime Minister Netanyahu.

\subsection{Attack Classes}

We break down attack classes into 4 groups based on Passeri's categorization: Hacktivism (H), Cyber Crime (CC), Cyber Espionage (CE), and Cyber Warfare (CW). These classes are not a universal set of accepted distinctions, and may sometimes overlap, but they describe the goal of a cyber attack fairly well. They correspond with the goals of a socio-political advantage (Hacktivism), a financial advantage (Cyber Crime), an informational advantage (Cyber Espionage), and an operational advantage (Cyber Warfare). The monthly attack volume of all 4 classes are illustrated in figure 5.25. Cyber crime accounts for most attacks reported, by a large margin. They peak just before and just after the 2016 US Presidential election, with a local minimum spanning 2.5 years (May 2015 - December 2018) between peaks. It is almost as if cyber criminals got tired and decided to take some time off. 


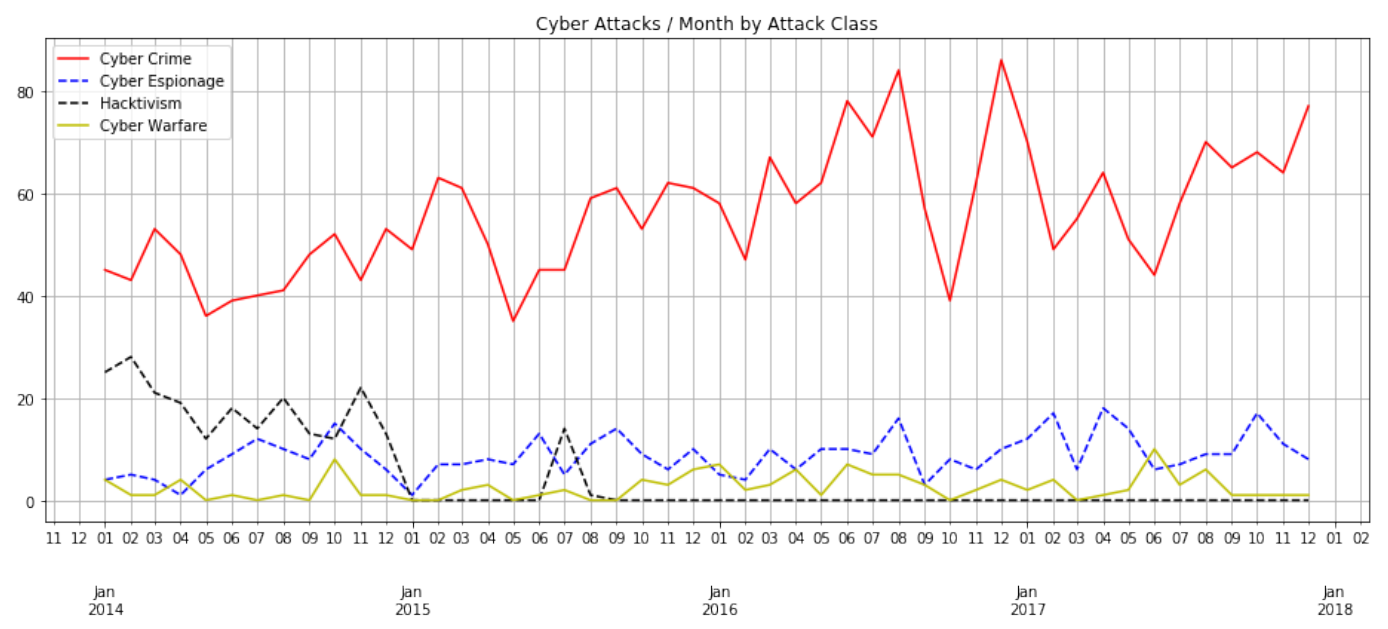

Figure 5.25: Attacks Timeline for All Attack Classes

Another interesting feature is that hacktivism reported plummets from a couple dozen attacks per month in the beginning of 2014 to zero reported hacktivism attacks since August 2015. This is probably not because of a lack of knowledge, as hacktivist activity is generally noticed in cyber security news. However, it may be difficult to classify an attack as related to hacktivism in certain situations. For example, there is an entry in the Hackmageddon data May 4, 2015, whose description reads, "A construction sign is hacked at the University of Montana, inviting users to "Smoke Weed Everyday"." The attack class is "Unknown". However, Montana had been a hotbed for political struggle over legalizing marijuana for many years [28]. This struggle came to a head in May, in which bipartisan legislation was introduced in the form of the Merkley-Daines amendment, which would allow doctors to recommend marijuana to veterans. It is reasonable to assume that the motivation was hacktivism, and that many other incidents are unnoticed, and confused for vandalism for vandalism's sake. Defacement is on the decline, and to some extent, DDoS attacks are also on decline. As a reminder, these numbers do not say anything about the number of individuals or machines affected; this means that it is possible that fewer attacks occur at a time when more widespread attacks occur, affecting more individuals and machines. 
Cyber warfare is very minimal and erratic, while cyber espionage is slowly increasing. Cyber warfare pits very sophisticated government-sponsored groups known as "advanced persistent threats" (APT's) against another country. The aggressor APT does not want to be disclosed in fear the country may seem malicious. Often, the victim country will not want to disclose an exposure for fear of appearing weak. Because the sophistication of these attacks, and their mutual concealment, events of cyber warfare may be the most concealed. Thus the Hackmageddon data likely only discloses a very small sample of the actual attacks countries pit against one another.

\subsection{Attack Vectors}

We show the most common attacks are in table 5.6. Their monthly totals are shown in figure 5.26. Account hijacking is the exfiltration of login credentials. Malware is unauthorized software running on a machine. Targeted attacks are a broad category of attacks that seek to compromise a system, often a piece of infrastructure, as when, in 2015, it was revealed that in 2013, a group of Iranian hackers had penetrated the online control system of a New York dam through a cellular modem [44]. SQL injection attacks occur when a user is able to directly manipulate SQL commands through a web form, thereby injecting malicious statements. DDoS is a distributed denial of service, in which many machines make requests to a server at once, overwhelming the server, making it unavailable. Defacement is hacking a website to change its content, and is a frequent result of hacktivist activity.

Unknown attacks peak in August 2016, the peak of the attacks on the US, and the global maximum of all attacks. Unknown attacks are those in which the technological means of the attack are not specified. The reason for it being unknown may be from the victim's true ignorance, or the victim may kept it secret for fear of the vulnerability being taken advantage of again. Account hijacking has been slowly 
increasing, and malware has increased dramatically, eclipsing unknown attacks as the dominant threat.

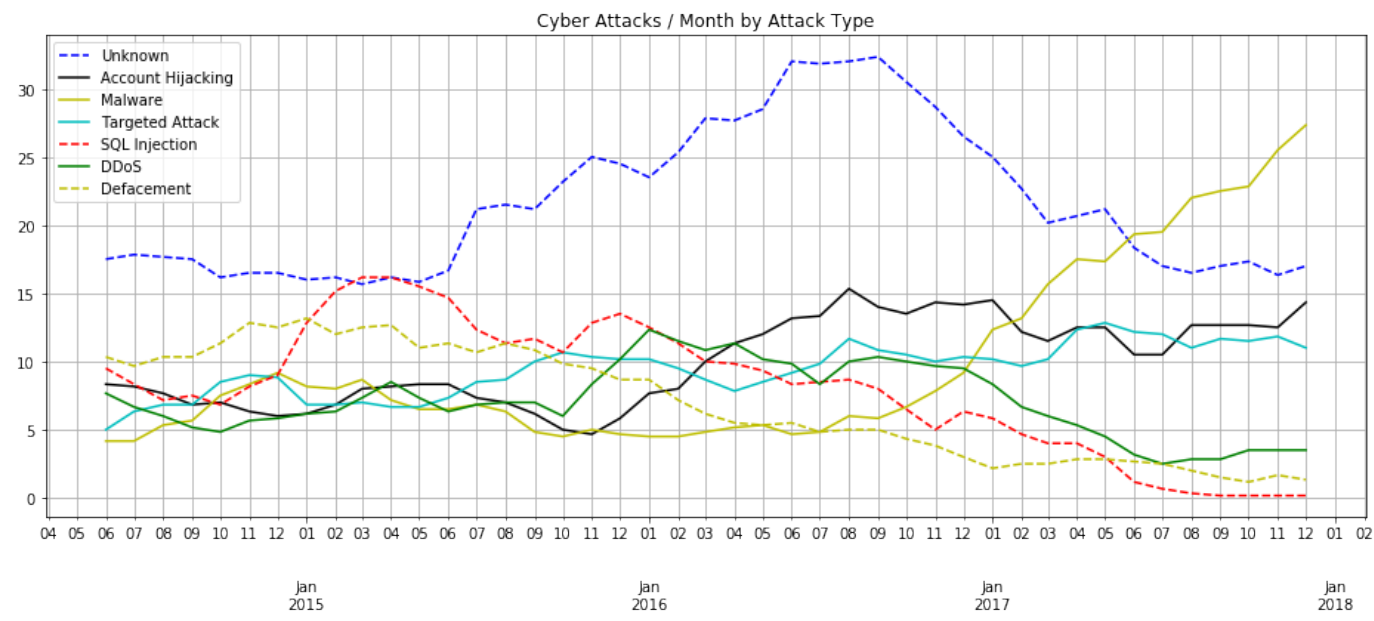

Figure 5.26: Attacks Timeline for All Attack Types, as a 6 month rolling average

\subsection{Apriori Results}

To answer the $3^{\text {rd }}$ question of this thesis, "What else can we learn about trends in cyber attacks?", we use the Apriori algorithm to discover frequent grouping of attack variables. To prepare a dataframe for the Aprior algorithm, each of the most popular values of 'TargetClass', 'TargetCountry', 'AttackClass', and 'Attack' are represented in a column of a pandas dataframe. The US is excluded because its overwhelming prevalence obscures other relationships. The columns are, 'TargetClass: Education', 'TargetClass: Government', 'TargetClass: Individuals', 'TargetClass: Industry', 'TargetClass: Finance', 'TargetClass: HealthCare', 'TargetClass: News', 'TargetClass: Org', 'Attack: DDoS', 'Attack: SQLInjection', 'Attack: AccountHijacking', 'Attack: Targeted', 'AttackClass: Hacktivism', 'AttackClass: CyberCrime', 'AttackClass: CyberEspionage', 'AttackClass: CyberWarfare', 'TargetCountry: GB', 'TargetCountry: CA', 'TargetCountry: AU', 'TargetCountry: RU', 'TargetCountry: 
KR', 'TargetCountry: FR', 'TargetCountry: UA', 'TargetCountry: JP', 'TargetCountry: IT', TargetCountry: DE', 'TargetCountry: PK', 'TargetCountry: BR', 'TargetCountry: TR', 'TargetCountry: NL', 'TargetCountry: SA', 'TargetCountry: India', 'TargetCountry: PH'. Each row represents 3 consecutive days from January 2014 December 2017, a total of 487 rows. The data value ' 1 ' is placed in a cell if an attack with the target value occurred within that specific 3 day period, and ' 0 ' otherwise. The results, in tables A.2, A.3, and A.4, use an abbreviated form of original column names: 'AC' for 'AttackClass', 'A': for 'Attack', 'TC' for 'TargetClass', and 'TCo' for 'TargetCountry'. We focus on support, which is simply the frequency that the union of the antecedents and consequents occurs in a dataset. A full explanation of the interpretation of Apriori results can be found in previous works [23] [30] [24]. The results in table A.2 imply that within 3 day intervals January 2014 - December 2017, the most features of attacks most frequently occurring are that they are financially destructive incidents (Cyber Crime) against the private sector (Industry). In any 3 day period over the time line, both occur $56 \%$ of the time. 


\begin{tabular}{|c|c|c|c|}
\hline Sector A & Sector B & $r$ & $\mathrm{p}$-value \\
\hline Individuals & Industry & -0.595 & 0.000 \\
\hline Individuals & Health Care & 0.562 & 0.000 \\
\hline Government & Industry & 0.548 & 0.000 \\
\hline Industry & Org & 0.476 & 0.000 \\
\hline Individuals & Finance & 0.446 & 0.001 \\
\hline Health Care & News & -0.360 & 0.010 \\
\hline Government & Individuals & -0.348 & 0.013 \\
\hline Government & Org & 0.346 & 0.014 \\
\hline Government & Finance & -0.345 & 0.014 \\
\hline Individuals & Org & -0.341 & 0.015 \\
\hline Industry & Finance & -0.339 & 0.016 \\
\hline Individuals & News & -0.326 & 0.021 \\
\hline Industry & Health Care & -0.283 & 0.046 \\
\hline Finance & Health Care & 0.258 & 0.070 \\
\hline Industry & News & 0.234 & 0.103 \\
\hline News & $>1$ & -0.233 & 0.104 \\
\hline Finance & Org & -0.225 & 0.116 \\
\hline Individuals & $>1$ & 0.199 & 0.167 \\
\hline Government & News & 0.151 & 0.294 \\
\hline News & Org & 0.150 & 0.298 \\
\hline Education & Individuals & -0.140 & 0.332 \\
\hline Government & Health Care & -0.137 & 0.342 \\
\hline Education & News & 0.099 & 0.496 \\
\hline Government & $>1$ & -0.092 & 0.523 \\
\hline
\end{tabular}

Table 5.3: Cross-industry attack volume correlations among the top 7 sectors ("TargetClass" field), sorted in ascending order by p-value. 


\begin{tabular}{|c|c|c|}
\hline Socio-Economic Factor & $r$ & p-value \\
\hline Population & 0.208 & 0.009 \\
\hline GDP & 0.179 & 0.027 \\
\hline Lower Confidence Interval of Happiness & 0.173 & 0.033 \\
\hline Happiness Score & 0.172 & 0.034 \\
\hline Upper Confidence Interval of Happiness & 0.170 & 0.036 \\
\hline Happiness Rank & -0.167 & 0.040 \\
\hline Log GDP per Capita & 0.175 & 0.041 \\
\hline Healthy Life Expectancy at Birth & 0.131 & 0.114 \\
\hline Generosity & 0.133 & 0.125 \\
\hline Delivery Quality & 0.408 & 0.147 \\
\hline Positive Affect & 0.109 & 0.199 \\
\hline Freedom To Make Life Choices & 0.098 & 0.247 \\
\hline Social Support & 0.092 & 0.274 \\
\hline People Can Be Trusted & 0.134 & 0.335 \\
\hline Confidence In Government & -0.062 & 0.488 \\
\hline Perception Of Corruption & -0.046 & 0.597 \\
\hline Democratic Quality & 0.026 & 0.929 \\
\hline Negative Affect & -0.004 & 0.962 \\
\hline
\end{tabular}

Table 5.4: Socio-Economic Correlates of Total Cyber Attack Volume Received 2014-2017 in ascending order by p-value. 


\begin{tabular}{|c|c|c|}
\hline Socioeconomic Factor & $r$ & p-value \\
\hline Population & 0.360 & 0.000 \\
\hline Healthy Life Expectancy at Birth & 0.299 & 0.000 \\
\hline Log GDP per Capita & 0.297 & 0.000 \\
\hline Lower Confidence Interval of Happiness & 0.281 & 0.000 \\
\hline Happiness Score & 0.274 & 0.001 \\
\hline Happiness Rank & -0.273 & 0.001 \\
\hline Upper Confidence Interval for Happiness & 0.268 & 0.001 \\
\hline GDP & 0.238 & 0.003 \\
\hline People Can Be Trusted & 0.383 & 0.005 \\
\hline Generosity & 0.145 & 0.095 \\
\hline Perception Of Corruption & -0.144 & 0.097 \\
\hline Delivery Quality & 0.408 & 0.147 \\
\hline Social Support & 0.121 & 0.150 \\
\hline Freedom To Make Life Choices & 0.111 & 0.192 \\
\hline Negative Affect & -0.075 & 0.372 \\
\hline Positive Affect & 0.076 & 0.374 \\
\hline Confidence In Government & 0.008 & 0.925 \\
\hline Democratic Quality & 0.026 & 0.929 \\
\hline
\end{tabular}

Table 5.5: Socio-Economic Correlates of Total Cyber Attack Volume Received 2014-2017 Excluding the US in ascending order by p-value. 


\begin{tabular}{|c|c|}
\hline Attack & Number of Recorded Attacks As \\
\hline Unknown & 1010 \\
\hline Account Hijacking & 507 \\
\hline Malware & 463 \\
\hline Targeted Attack & 449 \\
\hline SQL Injection & 349 \\
\hline DDoS & 339 \\
\hline Defacement & 317 \\
\hline
\end{tabular}

Table 5.6: The most common attack types. 


\section{Chapter 6}

\section{DISCUSSION}

In general, cyber attacks have a range of motivations, from monetary gain, political change, or simply spite, and other works have studied these [50]. In this study I cannot make a detailed conclusion of these motivations. Instead, I focus on the larger implications of each section. The main conclusions can be summarized in the following:

1. Countries with higher population, GDP, happiness, and other metrics that relate to resources are attacked somewhat more often.

2. There is an overall shift from attacking governments and businesses towards attacking individuals, even when political or financial gain is the object of the attack.

3. Malware has become the dominating threat in terms of number of attacks recorded in cyber security news.

The $1^{\text {st }}$ point is supported by previous cyber security research, in which GDP per capita is moderately correlated $(r=0.42, p<0.001)[33]$. The $2^{\text {nd }}$ point has not

been examined. The $3^{\text {rd }}$ point is supported by industry reports of a recent doubling of malware against IoT devices [20] and an overall decrease in DDoS attacks [11].

\subsection{Countries}

The data collected about attacks directed toward a country in particular is minuscule, and does not accurately estimate the true number of cyber attacks occurring. This is because most attacks are not recorded, and many of the recorded attacks are not 
publicized for fear of making the target person, corporation, country, or government appear weak. Nevertheless, the biases inherent in the collection of these attacks seem self-consistent. There are no observable changes in these biases, and a small sample of attacks against countries with more recorded attacks can inform us of how cyber attack volume truly changed, and what can be expected in the future.

The US is the most consistent with other studies. By this study, the US is the most attacked country, and this conforms to Kumar's account in "Approaches to understanding the motivations behind cyber attacks" [50]. Kumar also found that China was attacked with DDoS the $2^{\text {nd }}$ most. However, my findings indicate that China is only attacked by DDoS the $16^{\text {th }}$ most, and is attacked overall the $11^{\text {th }}$ most. As Kumar's findings use www . digitalattackmap. com [6] which directly records DDoS attacks as they happen, and mine rely on security reports, their numbers are much more accurate. However, from their limited recorded attacks, and the moderate correlation between attacks against China and Japan, we can say that their similar geography and economy make their cyber attacks tightly coupled. For instance, when the Yen and the Chinese stock market plummeted in January 2016 [2], China and Japan both saw their global maxima in cyber attacks 2014-2017 occur that month 5.13. This demonstrates the surprising power of incomplete data when sampled consistently.

Great Britain is the $2^{\text {nd }}$ most attacked country according to this study, and in 3 of 4 years, its annual maximum of attacks received occurs in November. This is again, using a very incomplete dataset, but it strongly implies that Great Britain's cyber attacks are cyclical, and are generally tied to the holiday shopping season.

The weak inverse relationship $(r=0.294, p=0.038)$ between attacks received by Pakistan and attacks received by Canada may seem perplexing. Because of its small sample size and surprising result, it is tempting to consider this finding in figure 5.12 
a fluke. However, accurate relationships have been found in other small sample sets, and the correlation is weak, so perhaps it is not so unusual after all.

On average, Pakistan and India seem periodic, maximizing on average in October in figure 5.9. They are geographically similar, and in mutual conflict, which is not surprising. This average is disproportionately influenced by the cyber attack volume of October 2014, which was a global maximum for both countries as implied by figure 5.14. Another interesting result in figure 5.14 is that the Indian elections, which only occurred once 2014-2017, occurred March through April [16], and one of India's 2 attack volume maxima occurred 3 months prior, in January. Four of January 2014's 8 attacks against India target the government directly. This maximization of cyber attacks received around 3 months prior to a national election also occurred in the 2016 US Presidential election. So it is reasonable to expect that 3 months before a contentious election, cyber attacks may increase.

\subsection{Sectors}

The clearest observation is that attacks against various industries are dereasing and attacks against individuals are increasing, as figure 5.20 illustrates. Attacks against individuals and industry are also the most correlated by absolute value, $(r=-0.595$, $p=0.000)$. There seems to be a systematic shift towards attacking individuals, the largest share of which are account hijacking (31\%), followed by malware (30\%). Of account hijacking against individuals, (14\%) were Twitter account hijacking. This is indicative of the types of trends to anticipate in the coming years.

Cyber attack volume against various industries and government sectors, as shown in figure 5.22, are very similar $(r=0.548, p=0.000)$. For the first 6 months they look like y-shifted versions of one another, and have the same sign of their first derivative; an increase in one means an increase in another. All this indicates that attackers 
target governments and the private sector at similar times, perhaps due to a tight coupling of politics and the economy of the US, the most attacked nation.

Attacks against several sectors $(>1)$ make up a small portion of attacks in figure 5.18, but are on the rise, quadrupling between 2014 and 2017. In addition to the graphs in section 5.2, the change in attacks against the most targeted sectors can be understood through table 6.1. For clarification, the "Organizations" category includes political parties and religious groups, which are neither private sector nor government agencies.

\begin{tabular}{|c|c|c|}
\hline Sector & 2014 January-June & 2017 July-December \\
\hline Individuals & $4 \%$ & $31 \%$ \\
\hline Industry & $34 \%$ & $18 \%$ \\
\hline Government & $19 \%$ & $10 \%$ \\
\hline Healthcare & $5 \%$ & $8 \%$ \\
\hline$>1$ & $2 \%$ & $8 \%$ \\
\hline Finance & $3 \%$ & $5 \%$ \\
\hline Organizations & $10 \%$ & $4 \%$ \\
\hline Education & $7 \%$ & $4 \%$ \\
\hline
\end{tabular}

Table 6.1: Contrast of the attacks by sector at the beginning half of 2014 and the last half of 2017.

Another way to profile these attacks against different sectors is to examine the type of attack most likely to occur in each sector, as shown in tables 6.2, 6.3 6.4

These data all imply that substantial portion of future attacks will target individuals, and this trend will continue to grow. 


\begin{tabular}{|c|c|}
\hline Attack & \% of Attacks Against Individuals \\
\hline Account Hijacking & $31 \%$ \\
\hline Malware & $30 \%$ \\
\hline Unknown & $9 \%$ \\
\hline Targeted Attack & $7 \%$ \\
\hline Malvertising & $5 \%$ \\
\hline Defacement & $2 \%$ \\
\hline Mobile Malware & $1 \%$ \\
\hline
\end{tabular}

Table 6.2: Types of attacks against individuals, by percentage.

\begin{tabular}{|c|c|}
\hline Attack & \% of Attacks Against Industry \\
\hline Unknown & $31 \%$ \\
\hline Account Hijacking & $10 \%$ \\
\hline SQL Injection & $10 \%$ \\
\hline Malware & $10 \%$ \\
\hline DDoS & $9 \%$ \\
\hline Targeted Attack & $7 \%$ \\
\hline Point of Sale Malware & $7 \%$ \\
\hline
\end{tabular}

Table 6.3: Types of attacks against industry, by percentage.

\subsection{Socioeconomic Factors}

From the results obtained, there are no strong correlations between any of the socioeconomic factors and an increase cyber attacks received, with a moderate correlation for only 3 factors. This difficulty is corroborated in Solano's "Socio-economic factors in cybercrime", which also does not find any strong correlations between socioeco- 


\begin{tabular}{|c|c|}
\hline Attack & \% of Attacks Against Governments \\
\hline Targeted Attack & $23 \%$ \\
\hline Defacement & $22 \%$ \\
\hline Unknown & $18 \%$ \\
\hline DDoS & $14 \%$ \\
\hline SQL Injection & $6 \%$ \\
\hline Malware & $5 \%$ \\
\hline Account Hijacking & $5 \%$ \\
\hline
\end{tabular}

Table 6.4: Types of Attacks Against Governments, by percentage.

nomic factors and cyber attacks received across all countries [64]. The top 3 socioeconomic factors that elevate the likelihood of cyber attacks are population $(r=0.360$, $p=0.000)$, healthy life expectancy at birth $(r=0.299, p=0.000)$, and $\log ($ GDP $)$ per capita $(r=0.297, p=0.000)$. Some of these categories that imply more cyber attacks are also at least weakly correlated to GDP closely tied to GDP. Healthy life expectancy is strongly correlated with $\log$ GDP per capita with $(r=0.811, p=0.000)$, and happiness score is weakly correlated with $\log$ GDP per capita with $(r=0.171$, $p=0.035)$. However, population is not correlated with log GDP per capita at all $(r=-0.032, p=0.714)$, indicating that attackers view people as a distinct resource on their own. More generally, the factors that matter are resources that malefactors can subvert. Those resources are the number of human beings and the money that a country holds. This makes sense, as the target of cyber attacks is often direct monetary gain, or account hijacking, which can then be used for monetary gain by using card payment information or through extorting the victims. Happier countries are attacked more often, with a correlation between happiness score and number of attacks received. Including the US, the correlation is $(r=0.293, p=0.001)$; excluding the US the correlation is $(r=0.183, p=0.035)$. In general, countries with more 
citizens, happier citizens, and more wealthy citizens are targeted more often.

\subsection{Attack Classes}

Over the span of the study 2014-2017, cyber crime has always dominated other attack classes, and continues to grow, as shown in 5.25. This asymmetry is likely because hacktivism is truly does represent a small slice of the pie, and cyber espionage and cyber warfare are not generally reported. Cyber crime is most likely a large source of cyber attacks, but the large gap between cyber crime and other attack classes is indicative of the culture of secrecy around cyber warfare and cyber espionage.

\subsection{Attack Vectors}

The peak of all attacks which occurred 3 months prior the US Presidential elections is also where cyber activity starts to change dramatically 5.26. Unknown attacks plummet, DDoS, SQL Injection, and Defacement attacks also taper off. Targeted attacks and account hijacking do not deviate much, but malware increases dramatically, so much so that December 2017 sees 5 times the number of malware reports as the average from the start of the study in 2014 until August 2016. Because malware is more associated with attacks against individuals, this larger trend implies as much about who is targeted as it implies about how they are being targeted. 


\section{Chapter 7}

\section{CONCLUSION}

Throughout this thesis I have analyzed cyber attack volume as a function of time, attack type, method of attack, target country, target sector, country to country relationships, socioeconomic factors, and have discovered tentative but meaningful correlations in various factors of the victims of cyber attacks. Furthermore, I accomplished this using only freely available data sources. I discovered that annually sampled socioeconomic statistics are difficult to correlate with cyber attack volume received, but GDP per capita and population play the strongest roles. Despite this tenuous relationship, the largest spikes in cyber attack volume are tied to socioeconomic events, namely contentious elections, political corruption, and stock market dives. The volume of future attacks will continue to increase, more often targeting individuals directly, affecting wealthier more populous nations including the US and China, and more often take the form of malware. The imminent ubiquity of the Internet of Things (IoT) will present new challenges to security as easier systems to compromise, a wider attack vector, and higher value objectives, such as home surveillance, and interference with physical systems, such as power plants, as well as consumer devices. Therefore, it is extremely important that the security research community knows as much as possible about trends in cyber attacks.

From the results which point to increased cyber attacks preceding contentious elections, I expect the US and the world to experience the most attacks of 2018 in August, preceding the US midterm elections. For the same reason, I also expect August 2020, preceding the US presidential election to have the highest volume of cyber attacks in the US and worldwide. The economy has been rocked recently, and that is often a predictor of cyber attacks. As President Trump has pulled out of the 
Paris Climate Accord, has considered pulling out of NAFTA, and recently imposed large tariffs on steel, aluminum, and other goods to allies in the EU, Canada, and Mexico [53]. The announcement of these tariffs recently dipped dramatically, as the Wall Street Journal notes, "Nasdaq was down 6.54 percent, marking their biggest weekly percentage falls since January 2016" [32]. Dramatic US economic changes like these will likely precipitate a higher volume of cyber attacks, as I occurred with the Chinese stock market crash described in section 6.1.

In the future, it would be very helpful obtain more accurate estimates of the numbers and locations of cyber attacks, either using a private database, similar to Carley's work [33], or by devising a way to reproduce similar results through open means. Additionally, correlating cyber attack volume with socioeconomic data on more than an annual basis, perhaps weekly, would bring greater clarity to how a changing socioeconomic climate directly affects cyber attack volume. Additionally, it may be helpful to store cyber attacks in a graph database, like Neo4j, which values relationships between entities over fast, uniform data access. In graph databases, entities are represented by vertices, and relationships are represented by edges. For instance, it is possible to use Neo4j's Cypher query language to detect the longest cycles of aggression in the following way:

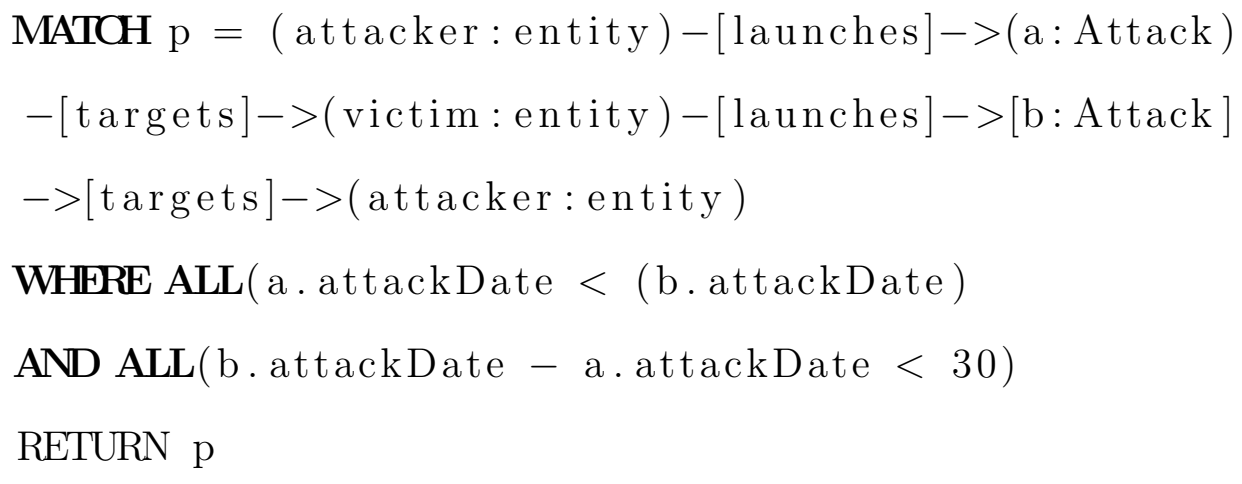

This of course, requires knowledge of the attacker, which is generally unknown in 
the data from Hackmageddon, and may also be unknown in the Symantec telemetry database used in Carley's work [33]. However, DDoS reports from Digital Attack Map [6] generally do identify at least the country of origin, which could be taken advantage of with a graph database. 


\section{BIBLIOGRAPHY}

[1] About the correlates of war project correlates of war. http://www. correlatesofwar.org/. (Accessed on 06/03/2018).

[2] Chinas stockmarket crashes again - open the door to green. https://www . economist. com/finance-and-economics/2016/01/04/chinasstockmarket-crashes-again. (Accessed on 05/27/2018).

[3] Country analysis, industry analysis - market risk assessment. https://www.eiu.com/home.aspx. (Accessed on 06/03/2018).

[4] Credit insurance group - credendo. https://www.credendo.com/. (Accessed on $06 / 03 / 2018)$.

[5] Cyber crime costs projected to reach $\$ 2$ trillion by 2019 . https://www .forbes. com/sites/stevemorgan/2016/01/17/cyber-crimecosts-projected-to-reach-2-trillion-by-2019/\#168014803a91. (Accessed on 05/19/2018).

[6] Digital attack map. http://www.digitalattackmap. com/\#anim=1\&color=0\& country=ALL\&list=0\&time=17661\&view=map. (Accessed on 05/27/2018).

[7] Downloads - world happiness report. http://worldhappiness.report/download/. (Accessed on 04/27/2018).

[8] Freedom house - championing democracy. https://freedomhouse.org/. (Accessed on 06/03/2018).

[9] Frequently asked questions — world happiness report. http://worldhappiness.report/faq/. (Accessed on 04/27/2018). 
[10] International labour organization.

http: //www.ilo.org/global/lang--en/index.htm. (Accessed on 06/03/2018).

[11] report-ddos-trends-q42017.pdf.

https://wWw.verisign.com/assets/report-ddos-trends-Q42017.pdf.

(Accessed on 06/09/2018).

[12] Russia used twitter bots and trolls to disrupt brexitvote - news - the times. https://www.thetimes.co.uk/article/russia-used-web-posts-todisrupt-brexit-vote-h9nv5zg6c. (Accessed on 05/17/2018).

[13] Transparency international - the global anti-corruption coalition. https://www.transparency.org/. (Accessed on 06/03/2018).

[14] Welcome to rsf website - rsf. https://rsf.org/en. (Accessed on 06/03/2018).

[15] World bank open data - data. https://data.worldbank.org/. (Accessed on 06/03/2018).

[16] eci.nic.in/eci_main1/current/press note ge-2014_05032014.pdf. http://eci.nic.in/eci_main1/current/Press\%20Note\%20GE2014_05032014.pdf, May 2014. (Accessed on 05/27/2018).

[17] Statistical appendix for chapter 2 whr. https ://s3. amazonaws. com/happinessreport/2015/StatisticalAppendixWHR3-April-16-2015.pdf, April 2015. (Accessed on 05/03/2018).

[18] Global device growth traffic profiles. https: //www.cisco.com/c/dam/m/en_us/solutions/service-provider/vni- 
forecast-highlights/pdf/Global_Device_Growth_Traffic_Profiles.pdf, 2016. (Accessed on 05/19/2018).

[19] Iot: number of connected devices worldwide 2012-2025 — statista. https://www.statista.com/statistics/471264/iot-number-ofconnected-devices-worldwide/, November 2016. (Accessed on 05/31/2018).

[20] Amount of malware targeting smart devices more than doubled in 2017 kaspersky lab. https://www.kaspersky.com/about/press-releases/2017_amount-ofmalware-targeting-smart-devices-more-than-doubled-in-2017, June 2017. (Accessed on 06/09/2018).

[21] Cisco's vni predicts global annual ip traffic to exceed - the network. https://newsroom.cisco.com/press-releasecontent?type=webcontent\&articleId=1853168, June 2017. (Accessed on $05 / 31 / 2018)$.

[22] Appendix1ofchapter2.pdf. https://s3.amazonaws.com/happinessreport/2018/Appendix1ofChapter2.pdf, March 2018. (Accessed on $05 / 17 / 2018)$.

[23] R. Agarwal, R. Srikant, et al. Fast algorithms for mining association rules. In Proc. of the 20th VLDB Conference, pages 487-499, 1994.

[24] M. Al-Maolegi and B. Arkok. An improved apriori algorithm for association rules. arXiv preprint arXiv:1403.3948, 2014.

[25] G. Aviles. How us political and socio-economic trends promotes hacktivist activity, 2015. 
[26] T. W. Bank. Gdp per capita, ppp (current international \$) - data. https://data.worldbank.org/indicator/NY.GDP.PCAP.PP.CD, 2016. (Accessed on 04/27/2018).

[27] T. Bergin and N. Layne. Special report: Cyber thieves exploit banks' faith in swift transfer network - reuters. https://www.reuters.com/article/uscyber-heist-swift-specialreport-idUSKCNOYBODD, May 2016. (Accessed on $05 / 15 / 2018)$.

[28] M. Bodley. Why montana is going backward on medical marijuana. https://www.nbcnews.com/news/us-news/why-montana-going-backwardmedical-marijuana-n410081, August 2015. (Accessed on 05/27/2018).

[29] D. Borak and K. Vasel. The equifax hack could be worse than we thought. http://money.cnn.com/2018/02/09/pf/equifax-hack-senatedisclosure/index.html, February 2018. (Accessed on 05/31/2018).

[30] S. Brin, R. Motwani, J. D. Ullman, and S. Tsur. Dynamic itemset counting and implication rules for market basket data. Acm Sigmod Record, 26(2):255-264, 1997.

[31] W. J. BROAD, J. MARKOFF, and D. E. SANGER. Stuxnet worm used against iran was tested in israel - the new york times. https://www.nytimes. com/2011/01/16/world/middleeast/16stuxnet.html?pagewanted=all, January 2011. (Accessed on 05/31/2018).

[32] S. Carew. Wall street nosedives as investors flee on trade war fears - reuters. https://www.reuters.com/article/us-usa-stocks/wall-streetnosedives-as-investors-flee-on-trade-war-fears-idUSKBN1GZ108?il=0, March 2018. (Accessed on 06/13/2018). 
[33] K. M. Carley, G. Mezzour, and L. Carley. Global mapping of cyber attacks. Technical report, CARNEGIE-MELLON UNIV PITTSBURGH PA PITTSBURGH United States, 2014.

[34] B. Chappell. U.s. says north korea 'directly responsible' for wannacry ransomware attack: The two-way: Npr. https:

//www.npr.org/sections/thetwo-way/2017/12/19/571854614/u-s-saysnorth-korea-directly-responsible-for-wannacry-ransomware-attack, December 2017. (Accessed on 05/31/2018).

[35] J. Clark. Why cyber security ignorance is bliss for iot hackers - computer business review. https://www. cbronline.com/internet-of-things/cybersecurity-ignorance-bliss-iot-hackers/, March 2017. (Accessed on $05 / 31 / 2018)$.

[36] J. Cohen. Statistical power analysis for the behavioral sciences 2nd edn, 1988.

[37] C. Debeck. The Correlates of Cyber Warfare: A database for the modern era. Iowa State University, 2011.

[38] L. Downes. A victory for medical marijuana - the new york times. https://takingnote.blogs.nytimes. com/2015/05/22/a-victory-formedical-marijuana/, May 2015. (Accessed on 05/27/2018).

[39] S. Erlanger. Britain votes to leave e.u.; cameron plans to step down - the new york times. https://www. nytimes. com/2016/06/25/world/europe/britainbrexit-european-union-referendum.html, June 2016. (Accessed on $05 / 21 / 2018)$.

[40] D. B. Figueiredo Filho, R. Paranhos, E. C. d. Rocha, M. Batista, J. A. d. Silva Jr, M. L. W. D. Santos, and J. G. Marino. When is statistical significance not significant? Brazilian Political Science Review, 7(1):31-55, 2013. 
[41] C. Forrest. $80 \%$ of iot apps not tested for vulnerabilities, report says techrepublic. https://www.techrepublic.com/article/80-of-iot-appsnot-tested-for-vulnerabilities-report-says/, January 2017. (Accessed on $05 / 31 / 2018)$.

[42] R. Gandhi, A. Sharma, W. Mahoney, W. Sousan, Q. Zhu, and P. Laplante. Dimensions of Cyber-Attacks: Cultural, Social, Economic, and Political. IEEE Technology and Society Magazine, 30(1):28-38, 2011.

[43] I. Gutzmer. Equifax announces cybersecurity incident involving consumer information - equifax. https://investor . equifax.com/news-andevents/news/2017/09-07-2017-213000628, September 2017. (Accessed on $05 / 31 / 2018)$.

[44] K. Hall. Iranian hackers targeted new york dam, had a quick nosy around the register. http://www.theregister.co.uk/2015/12/21/iranian_hackers_ target_new_york_dam/, December 2015. (Accessed on 05/28/2018).

[45] A. Hearn. Hacking risk leads to recall of 500,000 pacemakers due to patient death fears — technology — the guardian. https://www . theguardian.com/technology/2017/aug/31/hacking-riskrecall-pacemakers-patient-death-fears-fda-firmware-update, August 2017. (Accessed on 05/31/2018).

[46] J. Helliwell, R. Layard, and J. Sachs. World happiness report. 2012.

[47] Hiscox. https://www.hiscox.com/sites/default/files/content/2018-hiscox-cyberreadiness-report.pdf. https://www.hiscox.com/sites/default/files/content/2018-HiscoxCyber-Readiness-Report.pdf, February 2018. (Accessed on 05/30/2018). 
[48] I. Ilascu. Anonymous dumps email, facebook accounts and card data of israelis. https://news . softpedia.com/news/Anonymous-Dumps-Email-FacebookAccounts-and-Card-Data-of-Israelis-477710.shtml, April 2015. (Accessed on 05/21/2018).

[49] R. L. John Helliwell and J. Sachs. World happiness report 2015 — world happiness report. http://worldhappiness.report/ed/2015/, 2015. (Accessed on 04/27/2018).

[50] S. Kumar and K. M. Carley. Approaches to understanding the motivations behind cyber attacks. In Intelligence and Security Informatics (ISI), 2016 IEEE Conference on, pages 307-309. IEEE, 2016.

[51] S. Kumar and K. M. Carley. Ddos cyber-attacks network: Who's attacking whom. In Intelligence and Security Informatics (ISI), 2016 IEEE Conference on, pages 218-218. IEEE, 2016.

[52] D. K. Lambert and B. A. McCarl. Risk modeling using direct solution of nonlinear approximations of the utility function. American Journal of Agricultural Economics, 67(4):846-852, 1985.

[53] H. Long. Trump has officially put more tariffs on u.s. allies than on china - the washington post.

https ://www . washingtonpost. com/news/wonk/wp/2018/05/31/trump-hasofficially-put-more-tariffs-on-u-s-allies-than-onchina/?noredirect=on\&utm_term=.c82d97290af c, May 2018. (Accessed on 06/13/2018).

[54] M. G. Marshall. Polity iv project: Home page. http://www.systemicpeace.org/polity/polity4x.htm. (Accessed on 06/03/2018). 
[55] W. McKinney. Python for data analysis: Data wrangling with Pandas, NumPy, and IPython. " O'Reilly Media, Inc.", 2012.

[56] G. Mezzour, L. R. Carley, and K. M. Carley. Longitudinal analysis of a large corpus of cyber threat descriptions. Journal of Computer Virology and Hacking Techniques, 12(1):11-22, 2016.

[57] S. Morgan. Cybercrime damages $\$ 6$ trillion by 2021 . https://cybersecurityventures . com/hackerpocalypse-cybercrimereport-2016/, November 2017. (Accessed on 05/30/2018).

[58] S. D. S. Network. World happiness report — kaggle. https://www .kaggle.com/unsdsn/world-happiness/data, 2017. (Accessed on $04 / 27 / 2018)$.

[59] P. Passeri. About me hackmageddon. https://www . hackmageddon. com/about/. (Accessed on 04/20/2018).

[60] P. Passeri. Hackmageddon information security timelines and statistics. https://www.hackmageddon.com/. (Accessed on 05/27/2018).

[61] P. Passeri. 1-15 january 2018 cyber attacks timeline hackmageddon. https : //www . hackmageddon. com/2018/02/06/1-15-january-2018-cyberattacks-timeline/, February 2018. (Accessed on 05/06/2018).

[62] N. Sadan. Press note ge-2014_05032014.pdf. http://eci.nic.in/eci_main1/current/Press\%20Note\%20GE2014_05032014.pdf, May 2014. (Accessed on 05/17/2018).

[63] A. Sharma, R. Gandhi, Q. Zhu, W. R. Mahoney, and W. Sousan. A social dimensional cyber threat model with formal concept analysis and 
fact-proposition inference. International Journal of Information and Computer Security, 5(4):301-333, 2013.

[64] P. C. Solano and A. J. R. Peinado. Socio-economic factors in cybercrime: Statistical study of the relation between socio-economic factors and cybercrime. In 2017 International Conference On Cyber Situational Awareness, Data Analytics And Assessment (Cyber SA), pages 1-4, June 2017.

[65] I. Thompson. Hackers can turn web-connected car washes into horrible death traps. https://www.theregister.co.uk/2017/07/27/killer_car_wash/, July 2017. (Accessed on 05/31/2018).

[66] N. Watson. Mitigating losses from a trillion dollar cybercrime industry. https://www. entrepreneur.com/article/304971, November 2017. (Accessed on $05 / 30 / 2018)$.

[67] K. Zetter. An unprecedented look at stuxnet, the world's first digital weapon - wired. https://www.wired.com/2014/11/countdown-to-zero-day-stuxnet/, November 2014. (Accessed on 05/31/2018).

[68] K. Zetter. Inside the cunning, unprecedented hack of ukraine's power grid wired. https://www.wired.com/2016/03/inside-cunning-unprecedentedhack-ukraines-power-grid/, March 2016. (Accessed on 05/31/2018). 


\section{APPENDICES}

Appendix A

TABLES

Table A.1: The country-to-country correlation and p-values of the top 20 targets of cyber attacks, arranged in ascending order by p-value.

\begin{tabular}{|c|c|c|c|}
\hline Country A & Country B & $r$ & p-value \\
\hline TR & PH & 0.458 & 0.001 \\
\hline AU & IT & -0.366 & 0.009 \\
\hline IT & DE & -0.356 & 0.011 \\
\hline JP & CN & 0.339 & 0.016 \\
\hline US & JP & 0.326 & 0.021 \\
\hline SA & CN & 0.324 & 0.022 \\
\hline IT & PK & -0.318 & 0.024 \\
\hline US & CN & 0.309 & 0.029 \\
\hline RU & JP & 0.305 & 0.031 \\
\hline IL & PK & 0.297 & 0.037 \\
\hline CA & PK & -0.294 & 0.038 \\
\hline IN & PK & 0.289 & 0.042 \\
\hline IN & CN & 0.268 & 0.059 \\
\hline KR & NL & 0.268 & 0.059 \\
\hline US & TR & 0.265 & 0.063 \\
\hline IN & TR & 0.264 & 0.064 \\
\hline IL & IT & -0.264 & 0.064 \\
\hline US & DE & 0.251 & 0.079 \\
\hline
\end{tabular}




\begin{tabular}{|c|c|c|c|}
\hline Country A & Country B & $r$ & p-value \\
\hline US & $\mathrm{RU}$ & 0.246 & 0.086 \\
\hline FR & $\mathrm{PH}$ & 0.244 & 0.088 \\
\hline IT & $\mathrm{CN}$ & -0.241 & 0.091 \\
\hline $\mathrm{RU}$ & NL & 0.241 & 0.092 \\
\hline GB & $\mathrm{CA}$ & 0.23 & 0.109 \\
\hline GB & JP & -0.227 & 0.113 \\
\hline PK & NL & -0.216 & 0.131 \\
\hline RU & $\mathrm{UA}$ & 0.215 & 0.133 \\
\hline IT & $\mathrm{PH}$ & 0.215 & 0.134 \\
\hline RU & $\mathrm{KR}$ & 0.214 & 0.135 \\
\hline IN & FR & -0.213 & 0.137 \\
\hline US & INDIA & 0.212 & 0.14 \\
\hline $\mathrm{KR}$ & FR & -0.211 & 0.142 \\
\hline IN & IT & -0.21 & 0.143 \\
\hline US & $\mathrm{PH}$ & 0.209 & 0.145 \\
\hline IL & SA & 0.202 & 0.16 \\
\hline IL & $\mathrm{KR}$ & -0.197 & 0.17 \\
\hline $\mathrm{DE}$ & NL & 0.195 & 0.176 \\
\hline IL & $\mathrm{CN}$ & 0.194 & 0.177 \\
\hline UA & IT & 0.191 & 0.185 \\
\hline TR & $\mathrm{CN}$ & 0.19 & 0.186 \\
\hline PK & $\mathrm{CN}$ & 0.19 & 0.186 \\
\hline IN & SA & 0.188 & 0.191 \\
\hline $\mathrm{KR}$ & $\mathrm{CN}$ & 0.186 & 0.196 \\
\hline UA & PK & 0.185 & 0.197 \\
\hline
\end{tabular}




\begin{tabular}{|c|c|c|c|}
\hline Country A & Country B & $r$ & p-value \\
\hline GB & $\mathrm{AU}$ & 0.182 & 0.205 \\
\hline $\mathrm{AU}$ & IL & 0.178 & 0.217 \\
\hline $\mathrm{KR}$ & UA & 0.174 & 0.227 \\
\hline $\mathrm{AU}$ & JP & -0.174 & 0.228 \\
\hline IL & $\mathrm{DE}$ & -0.174 & 0.228 \\
\hline RU & $\mathrm{CN}$ & 0.172 & 0.231 \\
\hline IN & UA & 0.17 & 0.237 \\
\hline JP & NL & 0.169 & 0.241 \\
\hline UA & BR & -0.167 & 0.246 \\
\hline GB & $\mathrm{KR}$ & -0.167 & 0.247 \\
\hline GB & DE & 0.165 & 0.252 \\
\hline IL & $\mathrm{NL}$ & -0.165 & 0.253 \\
\hline $\mathrm{AU}$ & UA & -0.164 & 0.254 \\
\hline FR & JP & -0.161 & 0.265 \\
\hline NL & $\mathrm{PH}$ & -0.158 & 0.274 \\
\hline $\mathrm{CA}$ & NL & 0.157 & 0.275 \\
\hline IL & BR & 0.157 & 0.277 \\
\hline $\mathrm{CA}$ & $\mathrm{AU}$ & 0.156 & 0.279 \\
\hline GB & SA & 0.148 & 0.306 \\
\hline GB & CN & -0.147 & 0.308 \\
\hline BR & SA & 0.146 & 0.31 \\
\hline UA & $\mathrm{CN}$ & 0.146 & 0.312 \\
\hline $\mathrm{AU}$ & $\mathrm{DE}$ & 0.145 & 0.314 \\
\hline NL & $\mathrm{SA}$ & 0.143 & 0.322 \\
\hline $\mathrm{CA}$ & $\mathrm{KR}$ & -0.143 & 0.323 \\
\hline
\end{tabular}




\begin{tabular}{|c|c|c|c|}
\hline Country A & Country B & $r$ & p-value \\
\hline KR & TR & -0.14 & 0.331 \\
\hline IT & SA & -0.139 & 0.334 \\
\hline IN & DE & 0.138 & 0.34 \\
\hline TR & SA & 0.138 & 0.34 \\
\hline CA & IT & 0.134 & 0.354 \\
\hline GB & TR & -0.132 & 0.359 \\
\hline AU & SA & 0.131 & 0.366 \\
\hline CA & UA & -0.125 & 0.388 \\
\hline FR & NL & -0.124 & 0.393 \\
\hline JP & IT & -0.123 & 0.395 \\
\hline AU & RU & -0.123 & 0.396 \\
\hline GB & RU & -0.12 & 0.408 \\
\hline CA & SA & 0.116 & 0.421 \\
\hline GB & NL & -0.116 & 0.422 \\
\hline FR & TR & 0.114 & 0.429 \\
\hline CA & TR & -0.113 & 0.436 \\
\hline IN & BR & -0.112 & 0.44 \\
\hline IT & TR & 0.109 & 0.45 \\
\hline CA & BR & -0.109 & 0.452 \\
\hline RU & PK & -0.108 & 0.454 \\
\hline DE & TR & -0.106 & 0.466 \\
\hline PK & PH & -0.104 & 0.471 \\
\hline AU & KR & -0.103 & 0.477 \\
\hline KR & JP & 0.101 & 0.485 \\
\hline AU & FR & 0.098 & 0.5 \\
\hline
\end{tabular}




\begin{tabular}{|c|c|c|c|}
\hline Country A & Country B & $r$ & p-value \\
\hline IN & JP & 0.098 & 0.5 \\
\hline IN & RU & 0.097 & 0.501 \\
\hline $\mathrm{CA}$ & $\mathrm{DE}$ & 0.097 & 0.504 \\
\hline $\mathrm{CA}$ & $\mathrm{PH}$ & 0.095 & 0.512 \\
\hline US & FR & 0.091 & 0.528 \\
\hline $\mathrm{DE}$ & $\mathrm{PH}$ & -0.091 & 0.53 \\
\hline GB & IL & -0.09 & 0.533 \\
\hline RU & TR & 0.09 & 0.534 \\
\hline $\mathrm{KR}$ & $\mathrm{PH}$ & -0.09 & 0.535 \\
\hline $\mathrm{KR}$ & BR & -0.088 & 0.543 \\
\hline GB & INDIA & 0.088 & 0.545 \\
\hline RU & IL & 0.086 & 0.552 \\
\hline $\mathrm{CA}$ & FR & -0.086 & 0.555 \\
\hline $\mathrm{KR}$ & IT & -0.085 & 0.557 \\
\hline $\mathrm{AU}$ & PK & 0.082 & 0.571 \\
\hline GB & UA & -0.079 & 0.586 \\
\hline US & SA & 0.078 & 0.591 \\
\hline PK & $\mathrm{BR}$ & 0.078 & 0.592 \\
\hline $\mathrm{AU}$ & $\mathrm{BR}$ & -0.077 & 0.595 \\
\hline US & IT & -0.077 & 0.595 \\
\hline US & UA & -0.077 & 0.595 \\
\hline $\mathrm{DE}$ & $\mathrm{BR}$ & 0.076 & 0.598 \\
\hline IL & $\mathrm{PH}$ & -0.076 & 0.598 \\
\hline PK & TR & -0.076 & 0.6 \\
\hline FR & SA & -0.075 & 0.603 \\
\hline
\end{tabular}




\begin{tabular}{|c|c|c|c|}
\hline Country A & Country B & $r$ & p-value \\
\hline BR & TR & 0.072 & 0.618 \\
\hline IN & AU & 0.071 & 0.626 \\
\hline US & AU & 0.07 & 0.628 \\
\hline SA & PH & -0.069 & 0.634 \\
\hline DE & PK & -0.068 & 0.64 \\
\hline RU & SA & -0.065 & 0.654 \\
\hline FR & BR & -0.064 & 0.658 \\
\hline CA & RU & 0.058 & 0.688 \\
\hline AU & PH & -0.058 & 0.69 \\
\hline GB & IT & -0.057 & 0.695 \\
\hline JP & TR & 0.056 & 0.7 \\
\hline DE & SA & 0.055 & 0.703 \\
\hline GB & PK & -0.054 & 0.709 \\
\hline CA & CN & -0.053 & 0.713 \\
\hline US & IL & -0.052 & 0.717 \\
\hline FR & UA & 0.052 & 0.72 \\
\hline IN & PH & -0.052 & 0.721 \\
\hline RU & DE & -0.051 & 0.723 \\
\hline CA & IL & -0.051 & 0.723 \\
\hline RU & PH & -0.051 & 0.726 \\
\hline US & NL & 0.05 & 0.732 \\
\hline JP & SA & 0.049 & 0.735 \\
\hline KR & DE & -0.048 & 0.743 \\
\hline GB & BR & -0.045 & 0.758 \\
\hline PK & SA & -0.044 & 0.761 \\
\hline
\end{tabular}




\begin{tabular}{|c|c|c|c|}
\hline Country A & Country B & $r$ & p-value \\
\hline IN & NL & -0.041 & 0.775 \\
\hline US & PK & -0.04 & 0.784 \\
\hline UA & SA & 0.04 & 0.785 \\
\hline NL & CN & 0.039 & 0.788 \\
\hline BR & PH & 0.039 & 0.79 \\
\hline JP & PH & -0.037 & 0.801 \\
\hline IN & IL & 0.036 & 0.803 \\
\hline AU & TR & -0.035 & 0.808 \\
\hline JP & DE & -0.032 & 0.826 \\
\hline IT & NL & -0.031 & 0.829 \\
\hline UA & DE & -0.031 & 0.831 \\
\hline US & GB & 0.031 & 0.832 \\
\hline FR & CN & 0.028 & 0.846 \\
\hline US & CA & 0.027 & 0.852 \\
\hline CA & JP & -0.027 & 0.853 \\
\hline IT & BR & -0.026 & 0.856 \\
\hline AU & NL & 0.026 & 0.856 \\
\hline FR & DE & 0.025 & 0.861 \\
\hline US & BR & 0.025 & 0.862 \\
\hline BR & CN & 0.025 & 0.866 \\
\hline TR & NL & -0.023 & 0.872 \\
\hline IN & KR & 0.023 & 0.876 \\
\hline UA & JP & -0.022 & 0.878 \\
\hline BR & NL & 0.021 & 0.884 \\
\hline FR & PK & -0.019 & 0.896 \\
\hline
\end{tabular}




\begin{tabular}{|c|c|c|c|}
\hline Country A & Country B & $r$ & p-value \\
\hline RU & BR & -0.019 & 0.898 \\
\hline UA & PH & 0.018 & 0.902 \\
\hline IN & CA & -0.017 & 0.908 \\
\hline RU & FR & -0.016 & 0.915 \\
\hline RU & IT & 0.016 & 0.915 \\
\hline JP & BR & -0.015 & 0.916 \\
\hline FR & IT & 0.015 & 0.916 \\
\hline UA & NL & -0.014 & 0.921 \\
\hline IL & FR & -0.014 & 0.924 \\
\hline UA & TR & -0.014 & 0.924 \\
\hline KR & SA & -0.013 & 0.928 \\
\hline DE & CN & -0.012 & 0.936 \\
\hline AU & CN & 0.011 & 0.939 \\
\hline JP & PK & 0.011 & 0.942 \\
\hline GB & PH & 0.01 & 0.943 \\
\hline US & KR & 0.01 & 0.947 \\
\hline KR & PK & -0.008 & 0.954 \\
\hline PH & CN & 0.008 & 0.954 \\
\hline IL & JP & 0.008 & 0.957 \\
\hline IL & UA & 0.005 & 0.971 \\
\hline GB & FR & 0.002 & 0.988 \\
\hline IL & TR & -0.001 & 0.993 \\
\hline
\end{tabular}




\begin{tabular}{|l|l|l|c|c|c|c|c|c|}
\hline A & Cons & A.S. & C.S. & S & Conf & Lift & Lev & Conv \\
\hline TC: Indus- & AC: Cyber & 0.581 & 0.854 & 0.563 & 0.968 & 1.133 & 0.066 & 4.584 \\
\hline $\begin{array}{l}\text { TC: Gov- } \\
\text { ernment }\end{array}$ & AC: Cyber & 0.522 & 0.854 & 0.472 & 0.906 & 1.060 & 0.027 & 1.543 \\
\hline $\begin{array}{l}\text { AC: Cyber } \\
\text { Crime }\end{array}$ & Attack: & 0.854 & 0.448 & 0.411 & 0.481 & 1.074 & 0.028 & 1.064 \\
\hline $\begin{array}{l}\text { AC: Cyber } \\
\text { Crime }\end{array}$ & AC: Cyber & 0.854 & 0.429 & 0.390 & 0.457 & 1.064 & 0.024 & 1.051 \\
\hline $\begin{array}{l}\text { AC: Cyber } \\
\text { Espionage }\end{array}$ & Attack: & 0.429 & 0.448 & 0.382 & 0.890 & 1.988 & 0.190 & 5.019 \\
\hline
\end{tabular}

Table A.2: Top 5 apriori Rules associations for antecedent groups of size 1, in descending order by support. Column labels: "A" = antecedents, "Cons" = consequents, "A.S." = antecedent support, "C.S" = consequent support, "S"= support, "Conf" = confidence, "Lev" = leverage, "Conv" = conviction. Value labels: "AC" = Attack Class, "TC" = Target Class 


\begin{tabular}{|c|c|c|c|c|c|c|c|c|}
\hline $\mathrm{A}$ & Cons & A.S. & C.S. & $\mathrm{S}$ & Conf & Lift & Lev & Conv \\
\hline $\begin{array}{l}\text { AC: Cyber } \\
\text { Espionage, } \\
\text { AC: Cyber } \\
\text { Crime }\end{array}$ & $\begin{array}{l}\text { A: Tar- } \\
\text { geted }\end{array}$ & 0.390 & 0.448 & 0.347 & 0.889 & 1.987 & 0.172 & 4.998 \\
\hline $\begin{array}{l}\text { AC: Cyber } \\
\text { Crime, } \\
\text { TC: Gov- } \\
\text { ernment }\end{array}$ & $\begin{array}{l}\text { TC: Indus- } \\
\text { try }\end{array}$ & 0.472 & 0.581 & 0.337 & 0.713 & 1.227 & 0.062 & 1.460 \\
\hline $\begin{array}{l}\text { AC: Cyber } \\
\text { Crime, } \\
\text { TC: Gov- } \\
\text { ernment }\end{array}$ & $\begin{array}{l}\text { A: Tar- } \\
\text { geted }\end{array}$ & 0.472 & 0.448 & 0.273 & 0.578 & 1.292 & 0.062 & 1.310 \\
\hline $\begin{array}{l}\text { TC: In- } \\
\text { dustry, } \\
\text { AC: Cyber } \\
\text { Crime }\end{array}$ & TCo: GB & 0.563 & 0.357 & 0.271 & 0.482 & 1.348 & 0.070 & 1.240 \\
\hline $\begin{array}{l}\text { AC: Cyber } \\
\text { Crime, A: } \\
\text { Targeted }\end{array}$ & $\begin{array}{l}\text { TC: Indus- } \\
\text { try }\end{array}$ & 0.411 & 0.581 & 0.271 & 0.660 & 1.136 & 0.032 & 1.232 \\
\hline
\end{tabular}

Table A.3: Top 5 apriori Rules associations for antecedent groups of size 2 , in descending order by support. Column labels: "A" = antecedents, "Cons" = consequents, "A.S." = antecedent support, "C.S" = consequent support, "S"= support, "Conf" = confidence, "Lev" = leverage, "Conv" $=$ conviction. Value labels: "TCo" = Target Country. 


\begin{tabular}{|c|c|c|c|c|c|c|c|c|}
\hline A & Cons & A.S. & C.S. & $\mathrm{S}$ & Conf & Lift & Lev & Conv \\
\hline $\begin{array}{l}\text { AC: Cyber } \\
\text { Espionage, } \\
\text { AC: CC, } \\
\text { TC: Gov }\end{array}$ & $\begin{array}{ll}\text { A: Tar- } & \text { gated }\end{array}$ & 0.261 & 0.448 & 0.240 & 0.921 & 2.058 & 0.124 & 7.0150 \\
\hline $\begin{array}{l}\text { TC: I, AC: } \\
\text { CC, AC: } \\
\text { CE }\end{array}$ & $\begin{array}{l}\text { A: Tar- } \\
\text { geted }\end{array}$ & 0.248 & 0.448 & 0.226 & 0.909 & 2.031 & 0.115 & 6.076 \\
\hline $\begin{array}{l}\text { TC: I, AC: } \\
\text { CC, TC: } \\
\text { Gov }\end{array}$ & $\begin{array}{l}\text { A: Tar- } \\
\text { geted }\end{array}$ & 0.337 & 0.448 & 0.187 & 0.555 & 1.240 & 0.036 & 1.241 \\
\hline $\begin{array}{l}\text { TC: I, TC: } \\
\text { Gov }\end{array}$ & AC: $C C$ & 0.345 & 0.854 & 0.337 & 0.976 & 1.143 & 0.0421 & 6.123 \\
\hline $\begin{array}{l}\text { AC: } \quad \text { CE, } \\
\text { AC: } \quad \text { CC, } \\
\text { TC: Gov }\end{array}$ & TC: I & 0.261 & 0.581 & 0.179 & 0.685 & 1.179 & 0.027 & 1.330 \\
\hline
\end{tabular}

Table A.4: Top 5 apriori Rules associations for antecedent groups of size 3 , in descending order by support. Column labels: " $\mathrm{A}$ " = antecedents, "Cons" = consequents, "A.S." = antecedent support, "C.S" = consequent support, "S"= support, "Conf" = confidence, "Lev" = leverage, "Conv" $=$ conviction. Value labels: "TCo" = Target Country, "CC" = Cyber Crime, "E" = Espionage, "Gov" = Government, "I" = Industry . 